\title{
FACTORIZATION OF NUMBERS WITH PHYSICAL SYSTEMS
}

\author{
Dissertation \\ zur Erlangung des Doktorgrades \\ Dr. rer. nat. \\ der Fakultät für Naturwissenschaften \\ der Universität Ulm
}

vorgelegt von

WOLFGANG MERKEL

Institut für Quantenphysik

Universität Ulm

Leiter: Prof. Dr. Wolfgang P. Schleich

Ulm 2007 
Amtierender Dekan: Prof. Dr. Klaus-Dieter Spindler Erstgutachter: Prof. Dr. Wolfgang P. Schleich Zweitgutachter: $\quad$ Prof. Dr. Ferdinand Schmidt-Kaler Tag der Promotion: 6. Juni 2007 


\section{Contents}

List of Figures

vii

1 Introduction \& Motivation $\quad 1$

1.1 A glimpse on Shor's algorithm . . . . . . . . . . . . 4

1.2 Our approach to the factorization of numbers . . . . . . . 7

1.3 Relation to other work . . . . . . . . . . . 7

1.4 Organization of the present work .......... 8

2 Factorization with Gauss sums 9

2.1 An alternative representation of Gauss sums . . . . . . . . . 10

2.1.1 Sequence of individual contributions . . . . . . . . 11

2.1.2 Factorization made possible by the shape function $\mathcal{I}_{m}^{(r)} \quad$. 12

2.1.3 Factorization made possible by $\mathcal{W}_{m}^{(r)} \ldots \ldots \ldots 13$

2.2 Continuous sampling of Gauss sums . . . . . . . . . . . . . . . . 14

2.2.1 Discrimination of factors from non-factors . . . . . 14

2.2 .2 Scaling property . . . . . . . . . . . . 14

2.3 Discrete sampling of Gauss sums . . . . . . . . . . 17

2.3.1 Analytical expressions for the standard Gauss sum $G(\ell, N) \quad 17$

2.3.2 Factorization with $|G(\ell, N)|^{2} \ldots \ldots \ldots \ldots$

2.3.3 Factorization with real and imaginary part of $G(\ell, N)$. . 21

2.4 A second type of Gauss sum . . . . . . . . . . . . . . 22

2.4.1 Factorization based on complete Gauss sums . . . . . . 22

2.4.2 Factorization based on truncated Gauss sums . . . . . 25

2.4.3 Complexity of the factorization scheme . . . . . . . 33

2.5 Discussion . . . . . . . . . . . . . . 35 
3 Gauss sums arising from a chirped two-photon transition $\quad 37$

3.1 Chirped laser pulses . . . . . . . . . . . . . 37

3.1.1 Description of chirped pulses ............... 38

3.1.2 Applications of chirped pulses .......... 39

3.2 Chirped pulse excitation in a three-state ladder . . . . . . . . 40

3.2 .1 Model . . . . . . . . . . . . . . . 41

3.2 .2 Time evolution . . . . . . . . . . . . . 41

3.2.3 Perturbation theory in the weak field limit . . . . . . . 43

3.2 .4 Interference of quantum paths . . . . . . . . . . . . 45

3.2 .5 Origin of quadratic phases ........... . . . 45

3.2.6 Interference in time-frequency phase space . . . . . . 48

3.3 Chirped pulse excitation in a multistate ladder . . . . . . . . 50

3.3.1 Exact expression for the total excitation probability amplitude .................... 51

3.3.2 Asymptotic expansion of weight factors . . . . . . . 54

3.4 Candidate systems for an experimental realization . . . . . . . 54

3.5 Discussion . . . . . . . . . . . . . . 57

4 Gauss sums arising from laser-driven one-photon transitions $\quad 59$

4.1 Model ...................... . . 60

4.2 Floquet ladder . . . . . . . . . . . . . . . . . . 62

4.2.1 Excitation probability in the weak field limit . . . . . 63

4.2.2 Excitation probability for a chirped pulse . . . . . . . 64

4.2.3 Choice of modulation index ................. 65

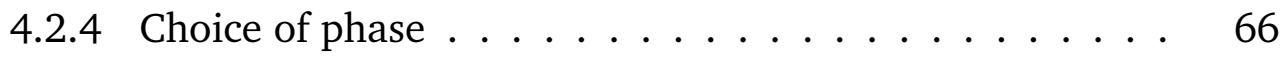

4.2 .5 summary ..................... 67

4.3 Pulse train . . . . . . . . . . . . . . . . 6 67

4.3.1 Excitation probability in the weak field limit . . . . . 68

4.3.2 Purely quadratic phases for a specific choice of parameters 68

4.4 Discussion . . . . . . . . . . . . . . 70

$\begin{array}{lll}5 & \text { Factorization with physical systems } & 71\end{array}$

5.1 Chirped multistate ladder . . . . . . . . . . . . 72

5.1.1 Continuous sampling of the signal . . . . . . . . 73

5.1 .2 Scaling property . . . . . . . . . . 73

5.2 Factorization based on Floquet ladder . . . . . . . . . . 74

5.2.1 Factorization with different types of sums . . . . . 76

5.2.2 Fluorescence signal at discrete arguments . . . . . . 78

5.3 Factorization with pulse train . . . . . . . . . . 79

5.4 summary ........................ 81 
6 NMR-Realizations of Gauss sums $\quad 85$

6.1 NMR experiment factors $N=157573 \ldots \ldots \ldots$. . . . . . . . . . . 86

6.2 A second NMR experiment . . . . . . . . . . . . . . . . . . . . 92

6.3 Discussion . . . . . . . . . . . . . . . . 92

$\begin{array}{lr}\text { Conclusions and Outlook } & 95\end{array}$

$\begin{array}{lll}\text { A Evaluation of Gauss sums } & 97\end{array}$

B Gauss sum for broad weight factor distributions 99

$\begin{array}{ll}\text { C Pocket factorizer } & 101\end{array}$

$\begin{array}{ll}\text { D Another type of Gauss sum } & 103\end{array}$

E Asymptotic expansion of the complementary error function $\quad 107$

E.1 Integration path for $a>0$ : decaying regime . . . . . . . . 110

E.2 Integration path for $a<0$ : oscillatory regime $\ldots \ldots 110$

E.3 Asymptotic expressions . . . . . . . . . . . . . . . 111

$\begin{array}{ll}\text { Bibliography } & 115\end{array}$

$\begin{array}{lr}\text { List of Publications } & 129\end{array}$ 
2.1 Factorization of $N=33 \ldots \ldots \ldots \ldots$

2.2 Factorization of $\tilde{N}=65$ by scaling the signal for $N=33 \ldots \ldots$

2.3 Factorization of $N=39$ and $41 \ldots \ldots$. . . . . . . . . 20

2.4 Factorization of $N=40$ and $42 \ldots \ldots \ldots 21$

2.5 Factorization with real and imaginary part of $G(\ell, N) \ldots 23$

2.6 Factorization of $N=1911$ with $\mathcal{A}_{N}^{(\ell-1)}(\ell) \ldots \ldots 26$

2.7 Truncating the summation range in the Gauss sum . . . . . . 27

2.8 Counting ghost factors . . . . . . . . . . . . . . . 29

2.9 Ghost factors in the complex plane . . . . . . . . . . . 31

2.10 Suppression of ghost factors . . . . . . . . . . . . . . . . 34

2.11 Optimal upper bound . . . . . . . . . . . . . 34

3.1 Three-state ladder system . . . . . . . . . . . . . . 41

3.2 Chirping a two-photon excitation in a three-state ladder . . . . 46

3.3 Origin of Gauss sums in phase space . . . . . . . . . . . . . 49

3.4 Extended ladder system ............... 50

3.5 Chirping a two-photon excitation through an equidistant manifold 53

3.6 Exact vs. approximated weight factors . . . . . . . . 55

4.1 Floquet ladder . . . . . . . . . . . . . . . . . . . . . 62

4.2 Weight factors $w_{m}(\phi) \ldots \ldots \ldots \ldots 6$

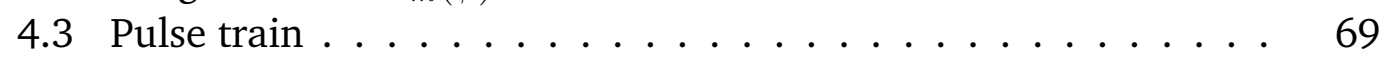

5.1 Factorizing $N=15 \ldots \ldots \ldots \ldots \ldots$

5.2 Scaling property . . . . . . . . . . . . . . 75

5.3 Floquet ladder: factorizing $N^{\prime}=21$ with $\mathcal{S}_{N^{\prime}}^{( \pm \pi / 2)} \ldots \ldots$. . . . . 77

5.4 Floquet ladder: factorizing $N^{\prime}=21$ with $\mathcal{S}_{N^{\prime}}^{(0)} \ldots \ldots \ldots$. . . . 78 
5.5 Floquet ladder: factorizing $N^{\prime}=21$ with $\mathcal{S}_{N^{\prime}}^{(\pi / 3)} \ldots \ldots \ldots \ldots 79$

5.6 Floquet ladder: factorizing $N^{\prime}=105 \ldots \ldots \ldots \ldots$

5.7 Pulse train: factorization of $N_{0}=1911 \ldots \ldots \ldots \ldots$

6.1 M. Mehring's NMR-experiment: pulse sequence . . . . . . . . 86

6.2 M. Mehring's NMR-experiment factorizes $N=157573 \ldots \ldots$. . 90

6.3 Probing the limits . . . . . . . . . . . . . . . . 91

6.4 D. Suter's NMR-experiment: pulse sequence . . . . . . . . . . . 93

6.5 D. Suter's NMR-experiment for $N=1911$ compared to theory . 93

C.1 Pocket factorizer . . . . . . . . . . . . . . . . . . 102

E.1 Integration paths in complex plane . . . . . . . . . . . . 109

E.2 Approximation of $\operatorname{erfc}\left(\zeta_{m}\right) \ldots \ldots \ldots \ldots \ldots$ 


\section{CHAPTER 1}

\section{Introduction \& Motivation}

Dw vxqulvh zh zloo dwwdfn wkh vpdoo Jdoolf yloodjh.

In history it has always been a major interest to keep the content of a message secret when exchanged between two parties [Sin00, Kip97]. It is interesting to analyze the history of cryptography in connection with the progress in physics and technology. In course of time the competition between the latter two counterparts has increased in intensity.

Originally, the application of cryptography was predominantly used for military or political purposes. Already Gaius Julius Caesar (102-44 B.C.) employed a simple cryptographic scheme to hide the content of his messages. This scheme is based on a simple substitution within the alphabet: Each letter of the plain text is replaced by the one which originates from a constant shift of the original letter. With this knowledge and Caesar's original shift of three letters the plain text can be decrypted by simple means ${ }^{1}$.

Once new technologies had been developed they had immediate application in cryptography. In World War II the German army used the electro-mechanical crypto-machine "Enigma" for encrypting top secret messages. One reason for the victory of the allied forces was certainly the ability of the cryptographs at Bletchley Park to decrypt the German communication once they got hold of an Enigma. The success of this endeavor relied on electro-mechanical devices which parallelized the underlying principles of the German Enigma [Sin00]. These "bombes" enabled the cryptographers to decipher German messages within a few hours. In some sense they can be viewed as predecessors of the modern computer. h Shortly after the end of the war the basis for a new era of computers was laid. In 1947 William Shockley, John Bardeen and Walter

\footnotetext{
${ }^{1}$ At sunrise we will attack the small Gallic village.
} 
Introduction \& Motivation

Brattain realized the first semi-conductor transistor (Nobel Prize 1956). This revolutionary technology rapidly evolved and gave rise to further miniaturization, which is connected to a dramatic increase of the number of elements on an integrated circuit. Consequently, computers became capable of solving increasingly complicated and extensive tasks. As a direct consequence the need for new cryptosystems arose. Of course, they should guarantee security against attacks with current and upcoming technology.

Until 1975 cryptography relied on symmetric keys where the same key is used for both encryption and decryption. Whitfield Diffie brought up the new idea to consider asymmetric schemes which consist of a publically accessible key and a second private key. Everybody who would like to safely transfer a message to a receiver, conventionally called Bob, has to use Bob's public key in order to generate the cipher from the plain text. Only Bob is able to recover the plain text by applying his private key.

Only two years later the three MIT scientists Ronald L. Rivest, Adi Shamir and Leonard Adleman proposed the first asymmetric encryption protocol $^{2}$ [Riv78]. The RSA protocol is based on the pair of multiplication and factorization, which is function and the associated inverse function. For the security of this scheme it is crucial that these two operations are strongly asymmetric in the required effort, thus acting like a "trapdoor" function: While the multiplication of two numbers is a computationally easy task, the inverse operation of factoring large numbers $N$ is a hard task in the sense that it requires exponential time. To illustrate this behavior we provide an estimation for the time required to factor a 100-digit number. According to Ref. [Ger05] this task would take on one of the best computers more than $1.4 \times 10^{10}$ years, which is a time of the order of the age of the universe.

Despite enormous efforts towards a solution of the factorization problem, so far no effective solution for an implementation on classical computers has been found. Based on these facts the RSA-protocol can be still regarded as safe. However, the size of the underlying numbers have to be adapted in accordance with the technological evolution of state-of-the-art computers.

Nowadays, cryptography basing on the RSA-system is applied by almost everyone, especially since the advent and spread of the personal computer and the world-wide web. The internet has turned to a huge market place where customers and companies interact in various ways. For example the transmission of private data, such as credit card numbers, is a delicate issue which requires utmost security. The security standards, however, solely rely on the fact that factorization is a hard task for computers which perform on the basis of classical physics.

\footnotetext{
${ }^{2}$ A similar protocol was developed a few years earlier by James Ellis, Clifford Cocks and Malcolm Williamson at the British GCHQ (Government Communications Headquarters), a topsecret organization which originated from the code-breakers at Bletchley Park. They were not allowed to publish their idea since the work of GCHQ underlied utmost secrecy.
} 
The next challenge to cryptography was ignited in 1982 by Richard Feynman when he came up with the seminal idea to study computers which perform on the basis of the laws of quantum physics [Fey82 $]^{3}$. The unique properties of quantum mechanics, such as the superposition principle and entanglement, can lead to an enhanced performance of such a quantum computer (QC) [Nie00, Ste05]. In the following years problems were identified for which quantum algorithms are more efficient than a solution on a classical computer. Besides few other problems factorization of numbers was identified as a candidate problem. In 1994 Peter Shor introduced his famous quantum factoring algorithm [Sho94, Sho97, Eke96], which requires only a polynomial number of operations on a quantum computer.

Needless to say, that an actual realization of this algorithm on a sufficiently powerful quantum computer would pose an immense threat to the security of data transfer. David DiVincenzo has formulated a set of general criteria [DiV00] which candidate quantum systems have to satisfy in order to realize a quantum computer.

The last years have witnessed enormous world-wide efforts in the quest of implementing quantum computation in various physical systems [Bou00] including nuclear magnetic resonance (NMR) [Van05], trapped atoms or ions [Cir95, Ste97, Lei03, Gul03, Roo04, Rie04, Tia05], cavity quantum electrodynamics [Rai01], and solid state systems like quantum dots [Ash96] and Josephson junctions [Mak01] in super conductors. Unfortunately, none of these settings satisfies the full set of DiVincenzo's criteria [USR04, EUR06]. However, combinations of above mentioned systems, such as spin systems in the solid state [Kan98], may represent a promising solution.

Currently, theoretical concepts for quantum information processing are far ahead compared to researchers engaged on the broad front of pushing forward experimental realizations. So far a wide variety of experimental demonstrations are at the proof-of-principle stage. Today the field of quantum computing is in a critical stage where its future crucially depends on making the step to the demonstration on a larger scale [USR04, EUR06].

The most promising perspective for QC is opened by the simulation of quantum dynamics [Llo96, Zal98, Jan03], an idea, which was already put forward by Richard Feynman [Fey82]. Quantum optical systems have already proven their potential to simulate solid-state systems [Por04] as impressively demonstrated by the example provided in Refs. [Jak98, Gre02].

In the next section 1.1 we set off by reviewing the working principle of Shor's algorithm. We discuss the key properties of our alternative approach to factorization in section 1.2 and identify connections to related work in section 1.3. Section 1.4 overviews the organization of the present work.

\footnotetext{
${ }^{3}$ Already in 1980 this idea was put forward by Yuri Manin.
} 
Introduction \& Motivation

\subsection{A glimpse on Shor's algorithm}

Shor's quantum factoring algorithm [Sho94, Sho97] is designed to perform on a quantum computer which obeys the laws of quantum mechanics. A combination of unique resources like superposition and entanglement allows to solve the problem of factorization of a large number $N$ in a time which scales only polynomially with the number of digits of $N$.

In the present section we summarize the key steps of Shor's algorithm ${ }^{4}$.

The central idea bases on the connection of the factorization problem to the problem of determining the order $r$ in modular exponentiation

$$
n^{r} \bmod N=1 \text {, }
$$

where $n$ is coprime to $N$, that is $\operatorname{gcd}(n, N)=1$. Once we have gained information on the order $r$ we employ the relation

$$
\left(n^{r / 2}-1\right)\left(n^{r / 2}+1\right) \bmod N=0
$$

to reveal the prime factor decomposition of $N$ by calculating greatest common divisors of $\left(n^{r / 2}-1\right)$ and $\left(n^{r / 2}+1\right)$ with $N$, respectively. Shor's algorithm provides an efficient tool to reveal the order $r$.

Initially the quantum computer is prepared in the state

$$
\left|\Psi_{0}\right\rangle=|0\rangle_{1}|0\rangle_{2}
$$

where the first register $|\ldots\rangle_{1}$ and the second register $|\ldots\rangle_{2}$ contain $y$ and $z$ quantum bits, respectively. We stress that the sizes of both registers have to be chosen in accordance with the number $N$ [Ger05].

In the first register we generate a uniform superposition

$$
\left|\Psi_{1}\right\rangle=\frac{1}{\sqrt{2^{y}}} \sum_{j=0}^{2^{y}-1}|j\rangle_{1}|0\rangle_{2}
$$

of all possible input states using a series of one-qubit Hadamard transformations.

This uniform superposition in the first register allows for exploiting the power of quantum parallelism. The big advantage of a quantum computer is based on the fact that unitary operations can be carried out simultaneously on many input states. We stress that this feature is in contrast to a classical computer where an operation has to be applied step-by-step for each single input.

The operation of modular exponentiation

$$
f\left(|j\rangle_{1}|0\rangle_{2}\right)=|j\rangle_{1}\left|n^{j} \bmod N\right\rangle_{2}=|j\rangle_{1}\left|f_{j}\right\rangle_{2}
$$

\footnotetext{
${ }^{4}$ For more detailed discussions we refer to [Ger05, Ste05, Lav03, Vol01]
} 
is performed simultaneously on all input states, thus creating the entangled state

$$
\left|\Psi_{2}\right\rangle=\frac{1}{\sqrt{2^{y}}} \sum_{j=0}^{2^{y}-1}|j\rangle_{1}\left|f_{j}\right\rangle_{2}
$$

where the results of the modular exponentiation are recorded in the second register.

A closer analysis of the state $\left|\Psi_{2}\right\rangle$ yields that the same entry $\left|f_{j}\right\rangle_{2}$ in the second register is always connected to entries $|k+m r\rangle_{1}$ in the first register, which are characterized by a period $r$ and an offset $k$. Making use of this observation we rewrite $\left|\Psi_{2}\right\rangle$ and arrive at

$$
\left|\Psi_{3}\right\rangle=\frac{1}{\sqrt{2^{y}}} \sum_{k=0}^{r-1} \sum_{m=0}^{Q-1}|k+m r\rangle_{1}\left|f_{k}\right\rangle_{2}
$$

where $k$ is an integer with $0 \leq k \leq r-1$. Moreover we have defined the number of states with the same entry $\left|f_{k}\right\rangle_{2}$ in the second register as

$$
Q=\left[\frac{1}{r}\left(2^{y}-1-k\right)\right]+1 .
$$

In general $Q$ can vary since $r$ does not always divide $2^{y}$ without residue. The Gauss bracket $[x]$ in Eq. (1.8) denotes the largest integer $\leq x$.

In order to achieve a clearer representation and to distill the periodic dependence of the entries in the first register we project ${ }^{5}$ the second register and arrive at

$$
\left|\Psi_{4}\right\rangle=\frac{1}{\sqrt{Q}} \sum_{m=0}^{Q-1}|k+m r\rangle_{1}
$$

where the order $r$ appears as a period in the entry of the first register.

But how can we gain information on $r$ from $\left|\Psi_{4}\right\rangle$ ? A quantum measurement is not suited as it only results in probabilities of finding a particular state rather than the state itself.

The answer to this central question is provided by the quantum Fourier transform

$$
\mathrm{QFT}|j\rangle_{1} \equiv \frac{1}{\sqrt{2^{y}}} \sum_{c=0}^{2^{y}-1} \exp \left[2 \pi i j \frac{c}{2^{y}}\right]|c\rangle_{1},
$$

which has the property that it moves the entry $j$ of the quantum state to a probability amplitude. When we apply the unitary QFT-operation to $\left|\Psi_{4}\right\rangle$ we find

$$
\left|\Psi_{5}\right\rangle=\frac{1}{\sqrt{Q 2^{y}}} \sum_{c=0}^{2^{y}-1} \exp \left[2 \pi i k \frac{c}{2^{y}}\right] \sum_{m=0}^{Q-1} \exp \left[2 \pi i m r \frac{c}{2^{y}}\right]|c\rangle_{1},
$$

\footnotetext{
${ }^{5}$ In principle this measurement can be postponed to the very end of the algorithm.
} 
Introduction \& Motivation

where the order $r$ appears in the probability amplitude.

In the following we distinguish two cases depending on $r$ : (i) $\mathrm{r}$ is a power of 2 and (ii) $r$ is not a power of 2 .

In the first exceptional case $(i)$ the number $Q=2^{y} / r$ of terms with the same entry $\left|f_{j}\right\rangle_{2}$ in the second register is exactly equal to an integer. We find that the second sum in $\left|\Psi_{5}\right\rangle$

$$
\frac{1}{Q} \sum_{m=0}^{Q-1} \exp \left[2 \pi i m r \frac{c}{2^{y}}\right]
$$

contributes only if there is an integer $d \in \mathbb{N}$ which satisfies the condition

$$
d=r \frac{c}{2^{y}} .
$$

In this case we find

$$
\left|\Psi_{5}\right\rangle=\sqrt{\frac{Q}{2^{y}}} \sum_{c=0}^{2^{y}-1} \exp \left[2 \pi i k \frac{c}{2^{y}}\right]|c\rangle_{1}
$$

and the probability $P(c)$ of finding the quantum computer in the state $|c\rangle_{1}$ reads

$$
P(c)=\left\{\begin{array}{cl}
1 / r & \text { for } c=d 2^{y} / r \\
0 & \text { else }
\end{array}\right.
$$

which is a peaked signal where adjacent maxima are separated by $Q=2^{y} / r$. From any non-zero measurement result $P(c) \neq 0$ we can infer information on the order $r$.

For the second case (ii) where $r$ is not a power of two, the ratio $2^{y} / r$ is obviously not an integer. Nevertheless, a measurement of $P(c)$ still allows to reveal information on the order $r$. If the number of qubits $y$ in the first register is sufficiently large, that is $N^{2}<2^{y}$, the second sum in Eq. (1.11) is reminiscent to a Delta function. In this case, however, the discrete probability distribution $P(c)$ never vanishes completely. But we still encounter maxima at values of $c$ which approximately correspond to integer multiples of $2^{y} / r$. By means of a continuous fraction expansion of $c / 2^{y}$ we can gain information on the order $r$.

This algorithm has to be repeated for various values of the basis $n$ until an even value for $r$ is obtained. Once we have found a pair of basis $n$ and even $r$ which satisfy Eq. (1.1), the prime factors of $N$ are revealed in a straightforward way by means of Eq. (1.2). We remark that the calculation of greatest common divisors can be efficiently implemented in a post-processing stage on a classical computer.

In summary, Shor's algorithm approaches the factorization problem via the connection to the problem of finding the order $r$ in modular exponentiation. The impressive speed-up inherent to this algorithm is based on the fact that 
the modular exponentiation operation is performed in parallel and leads to an entangled state where the order $r$ is imprinted as a period in the state vector of the quantum computer. The quantum Fourier transform moves $r$ from the entry of the quantum state to a probability amplitude which makes $r$ accessible in a measurement.

\subsection{Our approach to the factorization of numbers}

In the previous sections we have stressed the importance of the factorization problem in the context of cryptography and the security of world-wide communication. Moreover, we have identified the immense threat that would be imposed by the experimental realization of Shor's algorithm. Currently, the experimental progress encounters several challenging questions such that it is not clear whether such a powerful quantum computer could be ever realized.

In this work we pursue a different route and approach the factorization problem by combining aspects from number theory and quantum physics. Instead of attacking the factorization problem computationally, we show how the dynamics of laser-driven quantum systems lead to experimentally accessible quantities which are proportional to Gauss sums [Lan70, Dav80]. A Gauss sum can be viewed to emerge from the interference of multiple quantum paths where each path is weighted by a quadratic phase factor. This idea is the key to the present approach. The cyclic properties of Gauss sums are at the heart of our proposed schemes to factorize numbers.

The present approach to factorization solely bases on interference of multiple quantum paths. Our schemes perform without the quantum resource entanglement and do not capitalize on quantum parallelism. As a consequence, these schemes scale exponentially as a function of the number of digits of $N$. This is in contrast to Shor's algorithm which requires only a polynomial number of operations to factor a number $N$.

Nevertheless, this alternative approach provides interesting perspectives on the connection between physics and number theory [Mai07].

\subsection{Relation to other work}

Approaches relying on quantum interference had already been pursued before. For instance, in [Cla96] it was shown that the factors of an integer number $N$ can be revealed from the diffraction pattern of a Young $N$-slit interferometer.

Another proposal is based on wavepacket dynamics in anharmonic atomic or molecular potentials and the presence of quadratic phase factors in these systems [Mac02a, Mac02b]. Here the prime factors of an adequately encoded number $N$ can be extracted from the wave packet dynamics. 
Introduction \& Motivation

W. G. Harter [Har01] explains revival phenomena in wave packet dynamics in anharmonic potentials and quantum carpets of a free particle in a box from a group-theoretical point of view. Both systems feature quadratic spectra which may serve as quantum computing devices that perform automatically integer arithmetic. Moreover, the author provides a general explanation of the revival structure in quantum carpets by means of Farey sums.

In the beginning of the 20th century people pursued an alternative approach to solve the problem of factorizing numbers [Bei64]. At the University of California in Berkley father Derrick N. and son Derrick H. Lehmer constructed a machine for this purpose. The underlying principle is the implementation of modular arithmetic on a mechanical Gauss clock calculator. Derrick N. Lehmer generated tables of all prime numbers up to about 10.000 .000 using an early prototype of such a machine [Leh14]. These tables were actually the first which were accessible to mathematicians all over the world.

\subsection{Organization of the present work}

Chapter 2 provides the mathematical background for several factorization schemes basing on Gauss sums. Moreover, we show how to reveal the divisors of adequately encoded numbers from different types of Gauss sums. In chapter 3 we start with a short introduction to chirped pulses, which are characterized by non-linear phases. In this work we specialize on quadratic phases. Then we study a two-photon transition in a ladder system which contains an intermediate manifold of equidistant states. When we drive such a ladder with a chirped laser pulse we find that the resulting excitation probability amplitude is of the form of a Gauss sum. Chapter 4 is devoted to a discussion of two realizations of laser-driven one-photon transitions. For each case we show how Gauss sums arise in the excitation probability amplitude which is a quantity which might be measured afterwards. Main subject of chapter 5 is the demonstration of the factorization property of the previously proposed physical systems. We provide numerical simulations which underline the potential of various types of Gauss sums to factor numbers. Moreover, in chapter 6 we present the theoretical background of a recent NMR-experiment which implements Gauss sums. We conclude and address possible directions which might be subject of further investigations. 


\section{CHAPTER 2}

\section{Factorization with Gauss sums}

The present chapter is devoted to an analysis of the factorization properties intrinsic to different types of Gauss sums. Equipped with the results of this chapter we investigate in the following chapters 3 and 4 three physical systems which base on laser-driven transitions in specific ladder systems. All these realizations have in common that the lead to Gauss sums in the laser-induced excitation probability amplitude. In each case this quantity is experimentally accessible via measurements of the fluorescence signal from the excited state. For this reason we use the expression "signal" in the following when we have the absolute value (squared) of the Gauss sum in mind.

We study the generalized Gauss sum

$$
\mathcal{S}(\xi) \equiv \sum_{m=-\infty}^{\infty} \widetilde{W}_{m} \exp \left[2 \pi i\left(\frac{m}{A}+\frac{m^{2}}{B}\right) \xi\right]
$$

over linear and quadratic phase factors where we assume that $\xi$ is real-valued. Here, $A$ and $B$ denote two real numbers which we assume to fulfill a hierarchy $A \ll B$ and the weight factors $\widetilde{W}_{m}$ are slowly varying as a function of the summation index $m$.

We remark that such kind of sums have been studied in the context of fractional revivals [Par86, Ave89] in wavepacket dynamics in anharmonic potentials [Lei96a, Lei96b, Sch01]. Here the autocorrelation function is defined as the overlap of a time-evolved wavepacket with the initial wavepacket and is accessible in pump-probe experiments. Thus the autocorrelation function is the superposition of terms arising from each of the contributing bound states. Due to the time-evolution each contribution involves a phase factor which is proportional to the energy of the $m$-th bound state in the the anharmonic po- 
tential. The energy of each bound state is expanded in a series of terms in the quantum number $m$. When we take only linear and quadratic corrections into account we arrive at an autocorrelation function of the form of the Gauss sum in Eq. (2.1) where the argument $\xi$ is related to the propagation time. In this case $A$ corresponds to the classical period and $B$ stands for the revival time.

In order to uncover the deeper mathematical reason why Gauss sums have the intrinsic property to factorize numbers we first cast the sum of Eq. (2.1) into a slightly different form which paves the way to our factorization algorithm.

\subsection{An alternative representation of Gauss sums}

In the context of fractional revivals of wavepackets [Lei96a, Lei96b, Sch01] a method has been derived to rewrite the sum, Eq. (2.1), in an exact way as to bring out the features of $\mathcal{S}(\xi)$ typical for the different domains of $\xi$.

We now concentrate on arguments

$$
\xi_{r}=\ell A+\Delta \xi=\frac{q}{r} B+\varepsilon_{q / r} A+\Delta \xi
$$

that are close to a fraction $q / r$ of $B$ and are close to a large integer multiple $\ell$ of $A$. Both $q$ and $r$ are integer numbers and mutually coprime. The contribution $\varepsilon_{q / r} A$ is a correction term, since in general we have

$$
\ell A \neq \frac{q}{r} B
$$

According to Ref. [Lei96b] we can cast $\mathcal{S}\left(\xi_{r}\right)$ into the form

$$
\mathcal{S}\left(\xi_{r}\right)=\sum_{m=-\infty}^{\infty} \mathcal{W}_{m}^{(r)} \mathcal{I}_{m}^{(r)}(\Delta \xi)
$$

with the Gauss sums [Lan70, Dav80]

$$
\mathcal{W}_{m}^{(r)} \equiv \frac{1}{r} \sum_{p=0}^{r-1} \exp \left[2 \pi i\left(p^{2} \frac{q}{r}+p \frac{m}{r}\right)\right]
$$

and the shape functions

$$
\mathcal{I}_{m}^{(r)}(\Delta \xi) \equiv \int_{-\infty}^{\infty} d \mu \widetilde{W}(\mu) \exp \left\{2 \pi i\left[\left(\frac{\Delta \xi}{A}-\frac{m}{r}\right) \mu+\left(\varepsilon_{q / r}+\frac{\Delta \xi}{A}\right) \frac{A}{B} \mu^{2}\right]\right\}
$$

Here $\widetilde{W}(\mu)$ denotes the continuous extension of the weight factor distribution $\widetilde{W}_{m}$. 
According to Eq. (2.4) the generalized Gauss sum in the neighborhood of $\xi_{r}=\frac{q}{r} B+\varepsilon_{q / r} A+\Delta \xi$ consists of a sum of shape functions $\mathcal{I}_{m}^{(r)}$. Each term has the weight determined by the Gauss sum, Eq. (2.5). Here the fact if $r$ is even or odd is crucial[Sch01].

Indeed, from [Sch01] we recall the explicit result

$$
\left|\mathcal{W}_{m}^{(r)}\right|=\left\{\begin{array}{rlll}
\sqrt{1 / r} & \text { for } r \text { odd } & & \\
\sqrt{2 / r} & \text { for } r \text { even, } & r q / 2 \text { even and } & m \text { even } \\
0 & \text { for } r \text { even, } & r q / 2 \text { even and } & m \text { odd } \\
0 & \text { for } r \text { even, } & r q / 2 \text { odd and } & m \text { even } \\
\sqrt{2 / r} & \text { for } r \text { even, } & r q / 2 \text { odd and } & m \text { odd }
\end{array}\right.
$$

for the Gauss sum, Eq. (2.5). If $r$ is odd we have non-vanishing weights $\mathcal{W}_{m}^{(r)}$ for all values of the summation index $m$. However, if $r$ is even every second term in Eq. (2.4) vanishes.

\subsubsection{Sequence of individual contributions}

We now focus on the role of the shape function, Eq. (2.6) together with the Gauss sum. Both stand out most clearly for the example of a Gaussian weight function

$$
\widetilde{W}(\mu) \equiv \sqrt{\frac{1}{2 \pi \Delta n^{2}}} \exp \left[-\frac{1}{2}\left(\frac{\mu}{\Delta n}\right)^{2}\right]
$$

of width $\Delta n$. In this case we can perform the integral, Eq. (2.6), and find the explicit expression

$$
\mathcal{I}_{m}^{(r)}(\Delta \xi)=\tilde{\mathcal{N}}(\Delta \xi) \exp \left[-\frac{\left(\Delta \xi-\frac{m}{r} A\right)^{2}}{2 \sigma_{r}^{2}(\Delta \xi)}\right] \exp \left[-i \frac{\left(\Delta \xi-\frac{m}{r} A\right)^{2}}{2 \sigma_{i}^{2}(\Delta \xi)}\right]
$$

for the shape function. Here we have introduced the complex amplitude

$$
\tilde{\mathcal{N}}(\Delta \xi) \equiv \frac{1}{\sqrt{1-i 4 \pi \Delta n^{2}\left(\varepsilon_{q / r} A+\Delta \xi\right) / B}}
$$

and the widths

$$
\sigma_{r}^{2}(\Delta \xi) \equiv\left[\frac{1}{4 \pi^{2} \Delta n^{2}}+4 \Delta n^{2}\left(\frac{\Delta \xi+\varepsilon_{q / r} A}{B}\right)^{2}\right] A^{2}
$$

and

$$
\sigma_{i}^{2}(\Delta \xi) \equiv\left[\frac{B}{16 \pi^{3} \Delta n^{4}\left(\Delta \xi+\varepsilon_{q / r} A\right)}+\frac{1}{\pi} \frac{\Delta \xi+\varepsilon_{q / r} A}{B}\right] A^{2}
$$


of the real and the imaginary Gaussians.

According to Eq. (2.4) the sum $\mathcal{S}\left(\xi_{r}\right)$ contains the product $\mathcal{W}_{m}^{(r)} \mathcal{I}_{m}^{(r)}(\Delta \xi)$ of the Gauss sum $\mathcal{W}_{m}^{(r)}$ and the shape function $\mathcal{I}_{m}^{(r)}(\Delta \xi)$. The latter consists of the product of a complex-valued square root, a real and an imaginary Gaussian. Each term in the sum of the signal $\mathcal{S}\left(\xi_{r}\right)$ corresponds to such a product. We find that the $m$-th term is centered around $m A / r$.

However, each contribution appears with a weight function $\mathcal{W}_{m}^{(r)}$ which depends crucially on the parity of $r$. According to Eq. (2.7) if $r$ is odd all weights are non-vanishing and we find $r$ peaks within a unit $A$.

In contrast, for even values of $r$ every second weight factor vanishes. Therefore the signal $\mathcal{S}\left(\xi_{r}\right)$ exhibits only $r / 2$ peaks within a unit $A$. When the separation between two neighboring Gaussians is larger than their width $\sigma_{r}$ they do not overlap. In this case the sum over $m$, that is, over the individual Gaussians, separates into a sequence of Gaussians.

When neighboring non-vanishing terms $\mathcal{I}_{m}^{(r)}(\Delta \xi)$ and $\mathcal{I}_{m^{\prime}}^{(r)}(\Delta \xi)$ overlap, interferences between these terms arise. Then the phases of the complex Gaussian and the square root start to play an important role. Consequently, the sum $\mathcal{S}\left(\xi_{r}\right)$ exhibits a more complicated pattern.

\subsubsection{Factorization made possible by the shape function $\mathcal{I}_{m}^{(r)}$}

In the preceding section we have cast the sum $\mathcal{S}\left(\xi_{r}\right)$ into a sequence of complexvalued Gaussians. We now show, that this representation suggests a scheme to factorize numbers. This technique is directly applicable for odd numbers to be factorized.

For this purpose we return to the non-overlap criterion and recall from Eq. (2.11), that the width $\sigma_{r}$ of each Gaussian is different since it depends on $\Delta \xi$. The minimal width

$$
\sigma_{r}^{(\min )}=\frac{A}{2 \pi \Delta n}
$$

occurs for $\Delta \xi_{\min }$ defined by

$$
\Delta \xi_{\min }+\varepsilon_{q / r} A=0 .
$$

If $\varepsilon_{q / r}=0$ the quantity $\Delta \xi_{\min }$ of minimal width vanishes. Since the Gaussian with index $m=0$ also has its maximum at $\Delta \xi=0$, this Gaussian is of minimal width. Moreover, it has no overlap with neighboring ones provided $\sigma_{r}<\frac{A}{r}$. This condition puts the constraint

$$
\Delta n>\frac{r}{2 \pi}
$$

on the width $\Delta n$ of the Gaussian weight function $\widetilde{W}$, Eq. (2.8). 
According to Eq. (2.2), a vanishing correction term

$$
\varepsilon_{q / r}=\ell-\frac{q}{r} \frac{B}{A}=0
$$

corresponds to the condition

$$
\ell=q \frac{N}{r}
$$

where we have expressed the number $N \equiv B / A$ to be factorized in terms of the intrinsic scales $A$ and $B$ of our system.

Our initial ansatz, Eq. (2.2), implies that we consider the signal $\mathcal{S}\left(\xi_{r}\right)$ for arguments $\xi_{r}$ corresponding to integer multiples of $A$. Thus in Eq. (2.17) we have to choose the two coprime integers $q$ and $r$ such that $\ell$ is an integer. The condition is only met if $r$ corresponds to a factor of $N$. For $q=1$ we observe a well-localized Gaussian at the integer argument $\ell$ which corresponds to the first occurrence of the (prime) factor $N / r$. For $q>1$ this condition yields well localized Gaussians at arguments $\ell$ corresponding to the $q$-th multiple of the factor $N / r$. For odd $N$ we thus have the criterion: If the signal exhibits a maximum at the integer argument $\xi=\ell$ then $\ell$ corresponds to a prime factor or a multiple of a factor of $N$.

Before addressing the case of even $N$ we briefly discuss the case when $\ell$ is not a factor of $N$ and therefore $\varepsilon_{q / r}$ is non-zero. In this case the structure at $\Delta \xi=0$ is not a Gaussian of minimal width. Indeed, the widths of two neighboring Gaussians are so large, that they overlap considerably and the phase factors in $\widetilde{\mathcal{N}}$ and in the imaginary Gaussian, Eq. (2.9), lead to complicated interference structures. No clear fractional revival occurs at $\Delta \xi=0$.

\subsubsection{Factorization made possible by $\mathcal{W}_{m}^{(r)}$}

In contrast, for even values of $N$ we deal with a different situation.

So far we have utilized only the shape function $\mathcal{I}_{m}^{(r)}$ to factor odd numbers $N$. We now show that the Gauss sum $\mathcal{W}_{m}^{(r)}$ together with the shape function yields information on the factors of $N$.

Even though the condition Eq. (2.17) would allow for a maximum at the integer argument $\ell$, we still may observe a vanishing value of the signal. This behavior is due to the Gauss sum $\mathcal{W}_{m}^{(r)}$ which depends critically on the parity of the integers $r$ and $q$, see Eq. (2.7). We have seen that $\left|\mathcal{W}_{m}^{(r)}\right|$ vanishes for specific combinations of $r$ and $q$ thus leading to a suppression of the signal at certain integer arguments. For even $r$ and even $r q / 2$ the Gauss sum $\left|\mathcal{W}_{m}^{(r)}\right|$ vanishes for odd values of the summation index $m$, whereas for even $r$ and odd $r q / 2$ we find a vanishing Gauss sum $\left|\mathcal{W}_{m}^{(r)}\right|$ only for even values of the summation index $m$.

In this work we restrict ourselves to the factorization of odd numbers. Any given even number can be reduced in a straightforward fashion to an odd number by splitting of a power of 2 . We point out that the proposed factorization 
schemes are in principle also applicable to even numbers $N$. In this case we would gain information on the factors not only from the maxima of the signal $|\mathcal{S}(\xi)|^{2}$ but also from the minima of the signal.

In the next section we present an example which demonstrates the ability of Gauss sums in the context of factorizing numbers.

\subsection{Continuous sampling of Gauss sums}

For the following we restrict ourselves to a simplified representation of the Gauss sum where we choose the involved scales according to $A \equiv 1$ and $B \equiv N$. Thus we deal with the Gauss sum

$$
\mathcal{S}_{N}(\xi) \equiv \sum_{m} w_{m} \exp \left[2 \pi i\left(m+\frac{m^{2}}{N}\right) \xi\right]
$$

As before we assume that the weight factors are Gaussian distributed according to Eq. (2.8) with width determined by $\Delta n$.

In the following we show how to reveal information on the prime factors of the number $N$ from the absolute value squared $\left|\mathcal{S}_{N}(\xi)\right|^{2}$ of the Gauss sum $\mathcal{S}_{N}(\xi)$.

\subsubsection{Discrimination of factors from non-factors}

From the considerations of the preceding section 2.1 we identify factors of $N$ by analyzing the signal $\left|\mathcal{S}_{N}(\xi)\right|^{2}$ in the vicinity of integer arguments $\xi=\ell$. For most integers $\ell$ there is no combination of integer values $q$ and $r$ which satisfy the condition Eq. (2.17). In contrast, if such a pair of integres exists we expect a pronounced maximum of the signal $\left|\mathcal{S}_{N}(\xi)\right|^{2}$ at this integer argument $\xi=\ell$.

In Figure 2.1 we demonstrate how to reveal the prime factors of $N=33$ from the absolute value of the Gauss sum. We depict the signal $\left|\mathcal{S}_{N}(\xi)\right|^{2}$ as a function of the continuous argument $\xi$ and provide magnified insets in the vicinity of candidate prime factors. We identify distinct maxima of the signal at arguments corresponding to factors of $N$ whereas the signal at non-factor arguments does not show any peculiarities.

Moreover, we observe distinct maxima at non-integer arguments which are not relevant in the context of factorizing the number $N$. In Section 2.2.2 we will show that such maxima become important in the context of factorizing other numbers $\widetilde{N}$.

\subsubsection{Scaling property}

In this section we show that Gauss sums feature a remarkable scaling property. This property allows to factor a number $\widetilde{N}$ from a single realization of the signal 

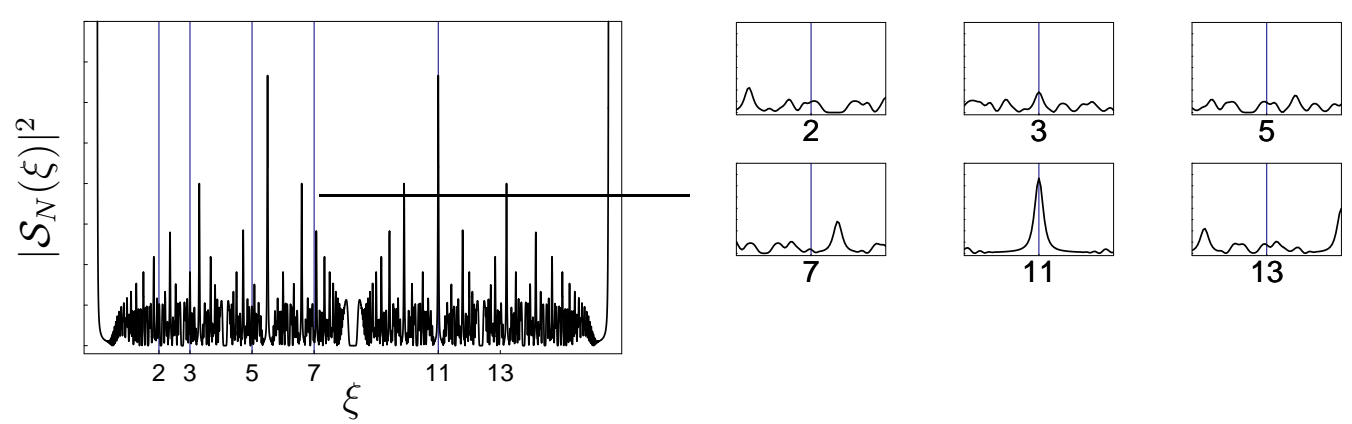

Figure 2.1: Factorization of $N=33$.

On the left we present an overview over the complete signal $\left|\mathcal{S}_{N}(\xi)\right|^{2}$ as a function of the continuous argument $\xi$. We indicate candidate prime factors by vertical lines. On the right we show the magnified signal in the vicinity of candidate primes. The pronounced maxima at the factors 3 and 11 are clearly visible. In contrast, at non-factor arguments the signal does not show any peculiarities. The width of the Gaussian distribution, Eq. (2.8), is $\Delta n=10$ so that the summation in the Gauss sum covers 81 terms.

$\left|\mathcal{S}_{N}(\xi)\right|^{2}$ acquired for the number $N$.

In the following we reconsider the signal $\left|\mathcal{S}_{N}(\xi)\right|^{2}$ at positions

$$
\xi=\frac{N}{\widetilde{N}} \tilde{\xi}
$$

where $\widetilde{N}$ represents the other number to be factorized.

With this substitution we rewrite the exponential of Eq. (2.18) according to

$$
\exp \left[2 \pi i\left(m+\frac{m^{2}}{N}\right) \xi\right]=\exp \left[2 \pi i\left(\frac{m}{\widetilde{N} / N}+\frac{m^{2}}{\widetilde{N}}\right) \tilde{\xi}\right]
$$

The resulting sum $\mathcal{S}_{\widetilde{N}}(\tilde{\xi})$ contains information on the factors of $\widetilde{N}$ for numbers $N$ and $\widetilde{N}$ being of the same order of magnitude. In this case they obey a hierarchy

$$
\frac{\widetilde{N}}{N} \ll \widetilde{N}
$$

Now the condition, Eq. (2.17), for the integer arguments $\tilde{\xi}=\tilde{\ell}$ corresponding to a factor of $\widetilde{N}$ reads

$$
\tilde{\ell}=q \frac{\tilde{N}}{r} .
$$

Hence, we have to analyze the original signal $\left|\mathcal{S}_{N}\right|^{2}$ in the vicinity of the integer arguments $\tilde{\xi}=\tilde{\ell}$.

In addition, we formulate the main result in other words. For the factorization of a number $N$ the signal $\left|\mathcal{S}_{N}(\xi)\right|^{2}$ has to be analyzed at arguments 

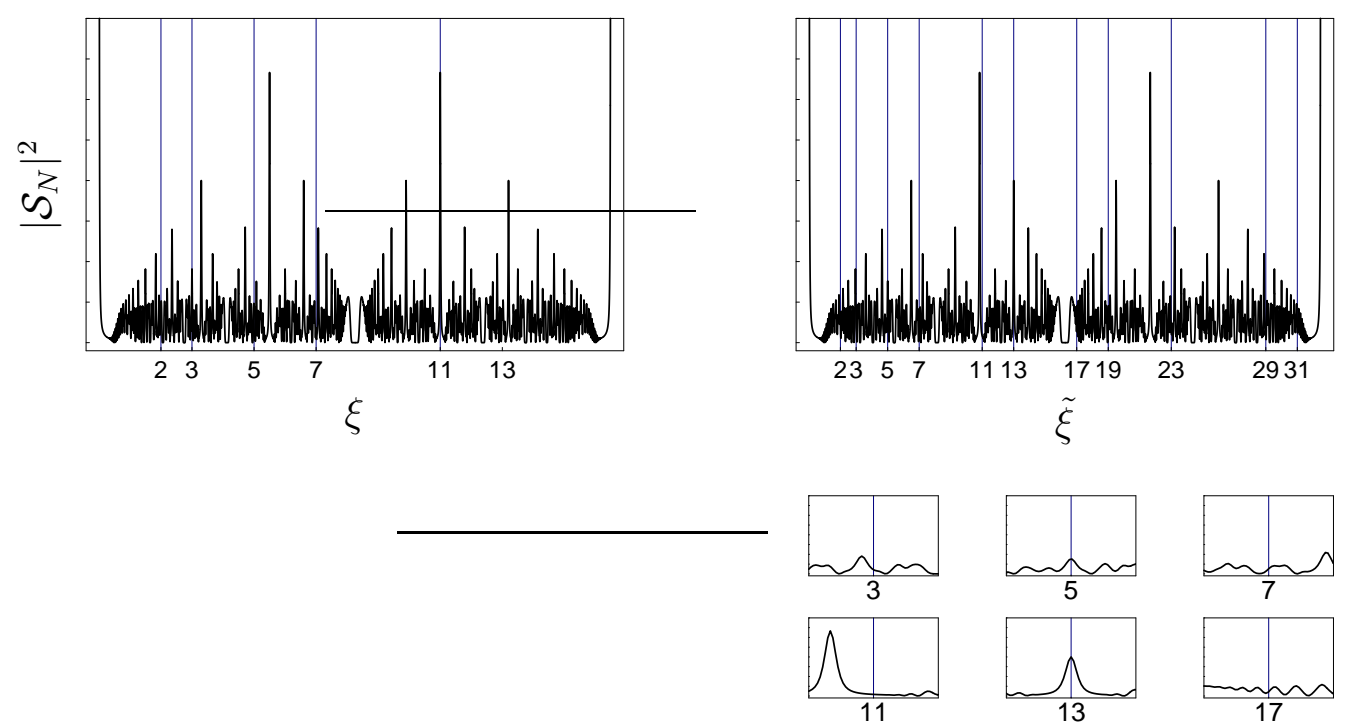

Figure 2.2: Factorization of $\widetilde{N}=65$ by scaling the signal for $N=33$.

By scaling the signal for $N=33$ (left), we obtain the signal corresponding to $\widetilde{N}=65$, as shown on the right. Candidate prime factors are depicted by vertical lines. The magnified insets clearly indicate the prime factors 5 and 13 of $\widetilde{N}$.

corresponding to integer multiples of the yardstick of scale 1 . For factorizing another number $\widetilde{N}$ we have to analyze the same signal using a yardstick which is characterized by a modified unit $N / \widetilde{N} \cdot 1$.

In Figure 2.2 we demonstrate this impressive feature with an example. We show how to reveal the prime factors of the number $\widetilde{N}=65$ starting from the signal $\left|\mathcal{S}_{N}(\xi)\right|^{2}$ originally acquired for the number $N=33$. Now maxima in the signal become important if they are positioned at integer arguments $\tilde{\xi}=\tilde{\ell}$. In contrast, maxima associated with the (prime) factors of $N$ are now irrelevant. The magnified insets show again the signal in the vicinity of integer arguments $\tilde{\xi}=\tilde{\ell}$.

Inspired by an idea by Prof. Stenholm we have generated a geometrical visualization of the scaling property intrinsic to Gauss sums. Starting point of the "pocket factorizer" is the signal $\left|\mathcal{S}_{N}(\xi)\right|^{2}$ for the number $N=51$. This tool offers a simple geometrical solution to the factorization of numbers $\widetilde{N}<100$. For factoring larger numbers $\widetilde{N}$ we would require a higher resolution of the original signal $\left|\mathcal{S}_{N}(\xi)\right|^{2}$ in the range of small arguments. A detailed explanation of the working principle can be found in Appendix C. 


\subsection{Discrete sampling of Gauss sums}

So far we have devised methods for revealing the prime factors of an adequately encoded number from a continuous signal $\left|\mathcal{S}_{N}(\xi)\right|^{2}$. However, also the knowledge of the signal at discrete integer argument $\xi=\ell$ suffices to gain information on the prime factor decomposition of $N$.

For integer arguments $\xi \equiv \ell$ the linear dependence in the phase factor drops out and we deal with purely quadratic phase factors

$$
\exp \left[2 \pi i\left(m+\frac{m^{2}}{N}\right) \ell\right]=\exp \left[2 \pi i m^{2} \frac{\ell}{N}\right] \text {. }
$$

In Appendix B we show that if we impose certain constraints on the weight factor distribution $w_{m}$ in the Gauss sum $\mathcal{S}_{N}(\ell)$ simplifies to the standard Gauss sum

$$
G(\ell, N)=\sum_{m=0}^{N-1} \exp \left[2 \pi i m^{2} \frac{\ell}{N}\right],
$$

We note that at this stage the argument $\ell$ and $N$ are not necessarily coprime. In Appendix A we introduce the main properties of the standard Gauss sum $G(a, b)$ which are relevant in the present work.

\subsubsection{Analytical expressions for the standard Gauss sum $G(\ell, N)$}

For the following we assume that we can split off a factor $p$ from the number to be factorized

$$
N=p \cdot q,
$$

where $q$ denotes the residual factor. Here we distinguish two cases of the integer argument $\ell$ :

(i) $\ell$ is not a factor of $N: \ell \neq p$, and

(ii) $\quad \ell$ is an integer multiple of a factor of $N: \ell=j p$ with $j \in \mathbb{N}$.

(i) No common factors between $\ell$ and $N$

We start our analysis with the case when the argument $\ell$ is not a factor of $N$. Here we directly apply the results of Appendix A and find

$$
G(\ell, N)=\left(\frac{\ell}{N}\right) G(1, N),
$$


where $\left(\frac{\ell}{N}\right)$ denotes the Legendre symbol which is discussed in Appendix A. We find

$$
G(\ell, N)=\left(\frac{\ell}{N}\right) \sqrt{N}\left\{\begin{array}{lll}
(1+i) & \text { for } & N \in \mathcal{M}_{0} \\
1 & \text { for } & N \in \mathcal{M}_{1} \\
0 & \text { for } & N \in \mathcal{M}_{2} \\
i & \text { for } & N \in \mathcal{M}_{3}
\end{array}\right.
$$

where the result depends on which set $\mathcal{M}_{k}$ the number $N$ belongs.

In general, any integer number $n$ is member of one of the sets

$$
\mathcal{M}_{k} \equiv\{n \mid n=4 s+k\},
$$

where $s$ is an integer and $k \in\{0,1,2,3\}$.

(ii) Argument $\ell$ is an integer multiple of a factor of $N$

We now turn to the case where the argument $\ell$ is an integer multiple of one of the factors of $N$, that is $\ell=j p$ with $j \in \mathbb{N}$. Note that here also the case of $\ell=N$ is included.

In this case the signal, Eq. (2.24), takes the form

$$
G(j p, N)=G(j p, q p)=\sum_{m=0}^{N-1} \exp \left(2 \pi i m^{2} \frac{j}{q}\right) .
$$

Using the fact that the phase factor $\exp \left(2 \pi i m^{2} j / q\right)$ is periodic with period $q$, we arrive at

$$
G(j p, q p)=p G(j, q)=p\left(\frac{j}{q}\right) G(1, q) .
$$

Hence, the value of the Gauss sum is given by

$$
G(j, q)=\left(\frac{j}{q}\right) p \sqrt{q}\left\{\begin{array}{lll}
(1+i) & \text { for } & q \in \mathcal{M}_{0} \\
1 & \text { for } & q \in \mathcal{M}_{1} \\
0 & \text { for } & q \in \mathcal{M}_{2} \\
i & \text { for } & q \in \mathcal{M}_{3}
\end{array} .\right.
$$

\subsubsection{Factorization with $|G(\ell, N)|^{2}$}

The results of Eqs. 2.27 and 2.31 for the standard Gauss sum $G(\ell, N)$ suggest another method to factorize numbers. For this purpose it is convenient to consider the normalized signal $|G(\ell, N)|^{2} / N^{2}$. This choice has the advantage that the signal values are now out of the range between 0 to 1 .

In the following we provide expressions for the modulus squared of the normalized Gauss sum for the two cases (i) and (ii) introduced in the previous section. 
(i) If the argument $\ell$ and the number $N$ do not share a common factor the value of the normalized signal

$$
\frac{1}{N^{2}}|G(\ell, N)|^{2}=\frac{1}{N}\left\{\begin{array}{lll}
2 & \text { for } \quad N \in \mathcal{M}_{0} \\
1 & \text { for } \quad N \in \mathcal{M}_{1}, \mathcal{M}_{3} \\
0 & \text { for } \quad N \in \mathcal{M}_{2} .
\end{array}\right.
$$

follows directly from Eq. (2.27). Moreover, we have applied the property of the Legendre symbol $\left|\left(\frac{a}{b}\right)\right|=1$.

(ii) In contrast, for arguments corresponding to integer multiples of a factor $p$ we find for the value of the normalized signal

$$
\frac{1}{N^{2}}|G(j p, N)|^{2}=p \frac{1}{N}\left\{\begin{array}{lll}
2 & \text { for } & q \in \mathcal{M}_{0} \\
1 & \text { for } & q \in \mathcal{M}_{1}, \mathcal{M}_{3} \\
0 & \text { for } & q \in \mathcal{M}_{2}
\end{array}\right.
$$

which follows together with the identity $(p \sqrt{q})^{2} \equiv N p$ directly from Eq. (2.31). Thus for arguments $\ell=j p$, which share a common factor with $N$, the value of the signal is critically determined by the residual factor $q=N / p$.

In the following we distinguish two cases depending on whether $N$ is odd or even.

$N$ is odd

First, we discuss the case when the number to be factorized is odd, that is $N \in \mathcal{M}_{1}$ or $\mathcal{M}_{3}$. Here also the constituting prime factors $p$ and $q$ are odd. Thus we deal with only one case when we reconsider the results of Eqs. 2.32 and 2.33 .

When we recall that $q$ as a factor of $N$ is always smaller than $N$ we find that the normalized signal features a larger value $(1 / q)$ at arguments corresponding to multiples of a factor $p$ of $N$ as compared to those arguments which correspond to non-factors $(1 / N)$.

In addition, we find another remarkable property which allows us to discriminate factors from non-factors: When we consider the normalized signal $|G(j p, N)|^{2} / N^{2}$ at the "first occurrence" $(j=1)$ of each factor $p$ we find that all datapoints $\left\{p,|G(p, N)|^{2} / N^{2}\right\}$ are aligned on the straight line

$$
\frac{|G(p, N)|^{2}}{N^{2}}=\frac{1}{N} p
$$

through the origin with slope $1 / N$. This line applies for all divisors of $N$, that is both prime factors and products of prime factors.

In Figure 2.3 we depict the normalized signal $|G(\ell, N)|^{2} / N^{2}$ of the Gauss sum of Eq. (2.24) for the numbers $N=39$ and $N=41$. Whenever the argument $\ell$ shares a common factor $p$ with $N$ the signal shows an enhanced value. For 

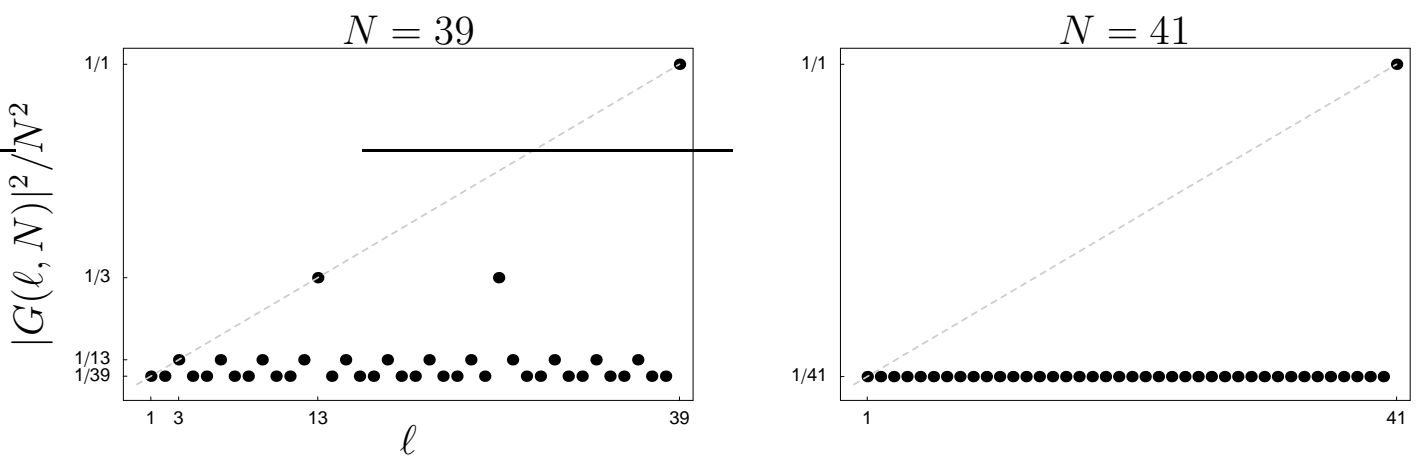

Figure 2.3: Factorization of odd numbers $N=39$ and 41 .

We depict the normalized Gauss sum $|G(\ell, N)|^{2} / N^{2}$, Eq. (2.24), for integer arguments $\ell$ for the odd number. $N=39$ (left) and the prime number $N=41$ (right). At divisors of $N$ the signal displays a maximum whereas this is not the case for non-factor arguments. For odd $N$ datapoints corresponding to divisors of $N$ are arranged on a straight line with slope $1 / N(N=39)$. For the prime number $N=41$ we observe an enhanced value of the signal only at $\ell=N$.

completeness we also show the case when $N$ is a prime number $(N=41)$. As expected, the normalized signal is 1 for $\ell=N$ whereas it is characterized by the suppressed value $1 / N$ for all other arguments $1<\ell<N$.

\section{$N$ is even}

When we consider the factorization of even numbers we also expect characteristic features of factors in a diagram which displays the normalized signal $|G(\ell, N)|^{2} / N^{2}$ as a function of integer arguments $\ell$.

Here the value of the signal of the normalized Gauss sum depends crucially on the set, Eq. (2.28), of $N$ and the residual factor $q$. We start with the case $N \in$ $\mathcal{M}_{0}$. We find from Eq. (2.27) that for arguments which do not share a factor with $N$ that the normalized signal takes on the value $2 / N$ since $N \in \mathcal{M}_{0}$. For arguments sharing a common factor with $N$ the signal value depends according to Eq. (2.33) on the membership of $q$ to a set $\mathcal{M}_{k}$.

We find that datapoints with $q \in \mathcal{M}_{0}, \mathcal{M}_{1}$ or $\mathcal{M}_{3}$ are aligned on two straight lines through the origin with slopes given by $1 / N$ and $2 / N$. For $q \in \mathcal{M}_{2}$ the normalized signal is zero.

Now we discuss the second case when $N$ belongs to $\mathcal{M}_{2}$. For arguments which do not share a common factor with $N$ we observe a zero signal. For the other case when $\ell$ and $N$ share a common factor the signal value again crucially depends on $q$. However, here the case of $q \in \mathcal{M}_{0}$ is not possible for $N \in \mathcal{M}_{2}$. Thus there is only one straight line with slope $1 / N$ which arises from odd values of $q$. The last case of $q \in \mathcal{M}_{2}$ results again in a vanishing signal. 

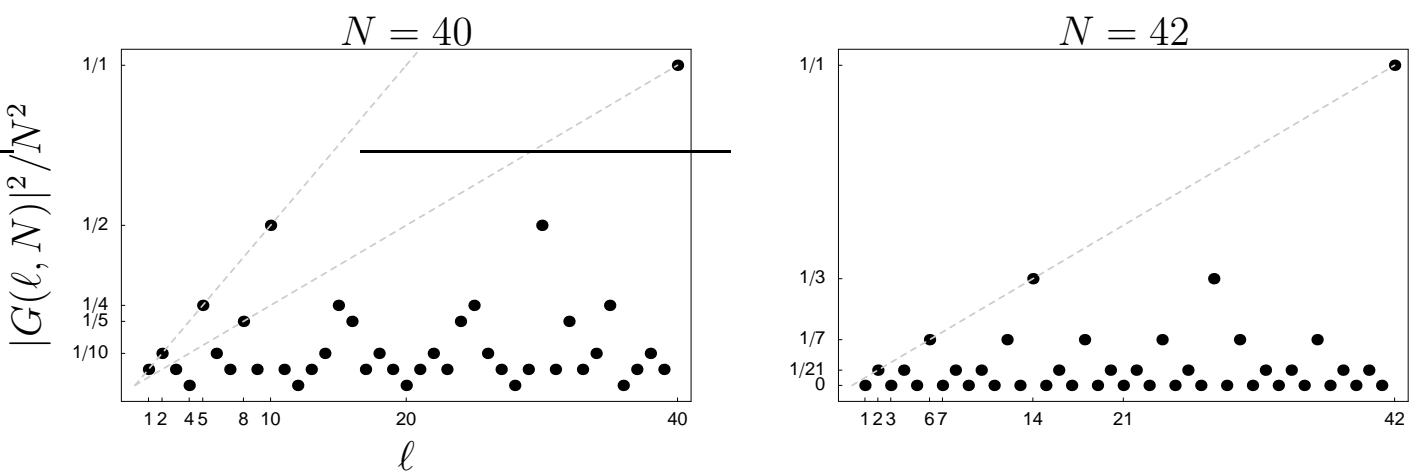

Figure 2.4: Factorization of $N=40$ and 42.

We depict the normalized Gauss sum $|G(\ell, N)|^{2} / N^{2}$, Eq. (2.24), for integer arguments $\ell$ for the even numbers $N=40 \in \mathcal{M}_{0}$ (left) and $N=42 \in \mathcal{M}_{2}$ (right). The values of the signal depend critically on the residual factor $q$ and are determined by Eqs. 2.32 and 2.33.

To illustrate these findings we provide the signal $|G(\ell, N)|^{2} / N^{2}$ for $N=40 \in$ $\mathcal{M}_{0}$ and $N=42 \in \mathcal{M}_{2}$ in Figure 2.4.

\subsubsection{Factorization with real and imaginary part of $G(\ell, N)$}

Apart from the behavior of the modulus squared of the standard Gauss sum $G(\ell, N)$ another indicator of factors of $N$ exists. We now show that under appropriate conditions they manifest themselves in the real and imaginary part of $G(\ell, N)$. Based on the results of section 2.3.1 we restrict our analysis to odd numbers, that is $N \in \mathcal{M}_{1}$ or $N \in \mathcal{M}_{3}$.

$N \in \mathcal{M}_{3}$

Such a number $N$ is always a product of two prime factors $p$ and $q$ which belong to different sets, that is $p \in \mathcal{M}_{1}$ and $q \in \mathcal{M}_{3}$, or vice versa. In the following we restrict our considerations to the first case. Depending on the argument the normalized Gauss sum $G(\ell, N) / N$ shows maxima in its real or imaginary part. Thus we can formulate the following criteria for determining the prime factors of $N$ : If the argument is equal to a multiple of $p$ the maxima appear in $|\operatorname{Im} G(\ell, N)| / N$ with amplitude $1 / \sqrt{q}$, whereas for multiples of $q$ the maxima are contained in $|\operatorname{Re} G(\ell, N)| / N$ or as zeros in $|\operatorname{Im} G(\ell, N)| / N$. As an example for this case we show in Figure 2.5 both real and imaginary part of the normalized Gauss sum for $N=35$ which is the product of the prime factors $5=4 \cdot 1+1 \in \mathcal{M}_{1}$ and $7=4 \cdot 1+3 \in \mathcal{M}_{3}$.

Of course, the same argumentation applies for the case where the roles of $p$ and $q$ are interchanged: $p \in \mathcal{M}_{3}$ and $q \in \mathcal{M}_{1}$. 
$N \in \mathcal{M}_{1}$

Such a number $N$ is the product of two primes $p$ and $q$ which belong to the same set, that is, we have to discuss the two cases i) $p, q \in \mathcal{M}_{1}$ and ii) $p, q \in \mathcal{M}_{3}$.

First we consider the case $i$ ) where both prime factors are members of $\mathcal{M}_{1}$. For all arguments we find non-zero values only in the real part of the normalized Gauss sum. Here multiples of the prime factors $p$ and $q$ appear with amplitudes $1 / \sqrt{q}$ and $1 / \sqrt{p}$, whereas for other arguments $n$ we find a considerable smaller value $|\operatorname{Re} G(\ell, N)| / N=1 / \sqrt{N}$. In Figure 2.5 we display the real and imaginary part of the normalized Gauss sum for $N=65$. Here it is only the real part which contains the full information on the prime factors of $N$.

Now we turn to the case ii) where both prime factors are member of $\mathcal{M}_{3}$. Here we find that the imaginary part of the normalized Gauss sum is sufficient to pin down the prime factors of $N$. Multiples of $p$ and $q$ are weighted with amplitudes $1 / \sqrt{q}$ and $1 / \sqrt{p}$ in $|\operatorname{Im} G(\ell, N)| / N$, whereas the imaginary part vanishes for arguments $n$ which do not share a common factors with $N$. For $N=33$ we present real and imaginary part in Figure 2.5. We clearly recognize the distinct maxima at multiples of $p=3 \in \mathcal{M}_{3}$ and $q=11 \in \mathcal{M}_{3}$ in the imaginary part.

\subsection{A second type of Gauss sum}

In the context of factorization it is also interesting to study another type of Gauss sum

$$
\sum_{m} \exp \left(2 \pi i m^{2} \frac{N}{\ell}\right)
$$

where the roles of argument $\ell$ and number $N$ to be factorized are interchanged in contrast to the previous Gauss sum $\mathcal{S}_{N}$.

To gain information on the divisors of a number $N$ we analyze factorization interference patterns where we depict the absolute value of the Gauss sum as a function of integer values of $\ell$. For a complete factorization interference pattern the argument $\ell$ scans through all numbers from 1 to $\sqrt{N}$.

\subsubsection{Factorization based on complete Gauss sums}

To start our analysis we first consider the normalized complete Gauss sum

$$
\mathcal{A}_{N}^{(\ell-1)}(\ell)=\frac{1}{\ell} \sum_{m=0}^{\ell-1} \exp \left(2 \pi i m^{2} \frac{N}{\ell}\right),
$$

where the superscript $(\ell-1)$ accounts for the fact that the summation covers a complete period ranging from $m=0$ to $\ell-1$. For $\ell$ being a factor of $N$ all 

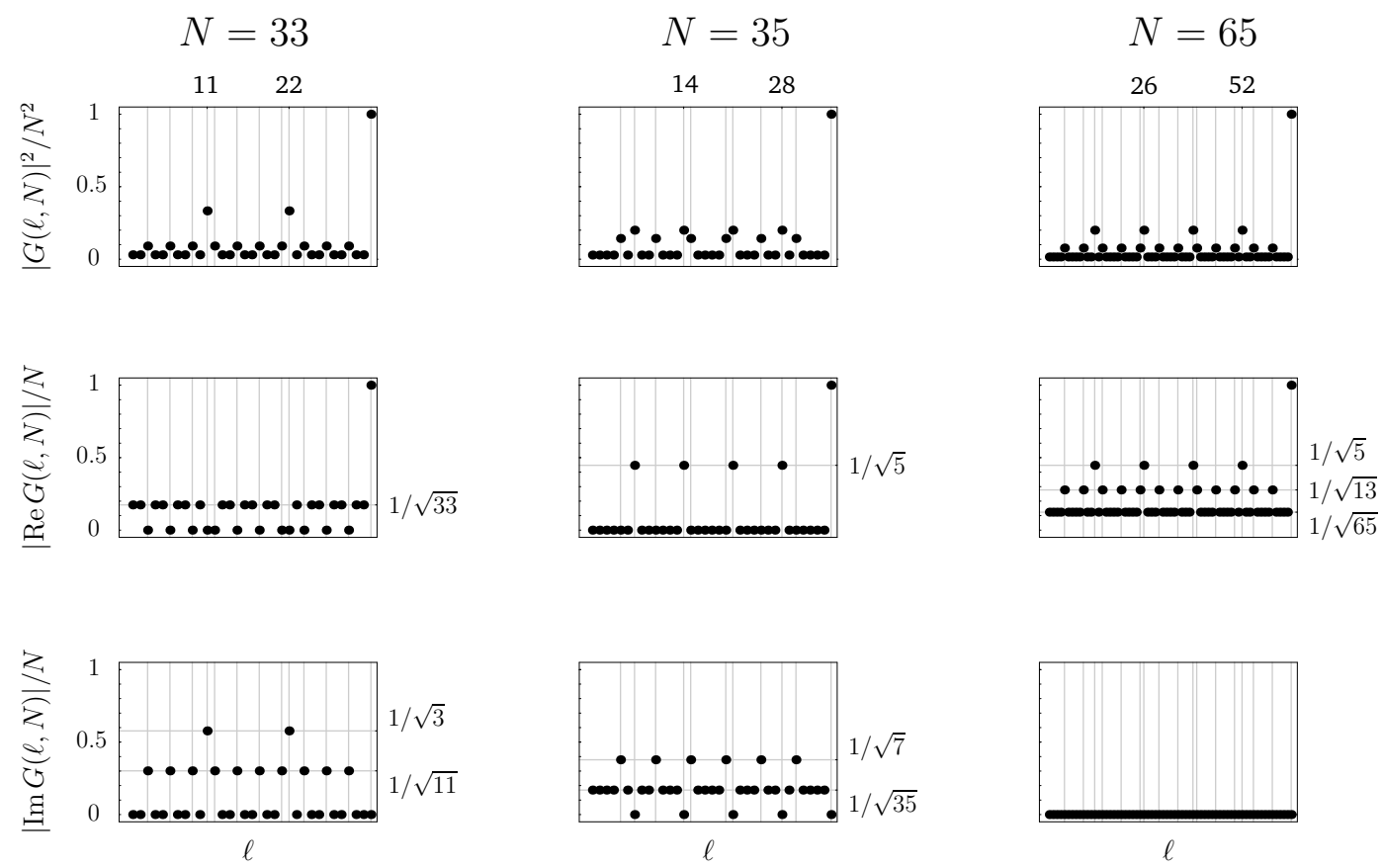

Figure 2.5: Normalized Gauss sum $G(\ell, N) / N$ for $N=33=4 \cdot 8+1 \in \mathcal{M}_{1}$ (left column), $N=35=4 \cdot 8+3 \in \mathcal{M}_{3}$ (middle column) and $N=65=4 \cdot 16+1 \in \mathcal{M}_{1}$ (right column). From top to bottom, we show modulus squared, real and imaginary part of $G(\ell, N) / N$, Eq. (2.24). For $N=33$ only multiples of both factors 3 and 11 show non-zero imaginary parts. For $N=35$ only multiples of the factor 7 yield a non-zero real part. The imaginary part exhibits zeros for multiples of the factor 7 and maxima for multiples of 5 . For $N=65$ the full information is contained in the real part, whereas the imaginary part does not contain any information on the prime factors of $N$.

Reprinted figure with permission from W. MERKEL, O. CRASSER, F. HAUG, E. LUTZ, H. Mack, M. Freyberger, W. P. Schleich, I. Sh. Averbukh, M. Bienert, B. Girard, H. MAIER and G. G. PAUlus, "Chirped pulses, Gauss sums and the factorization of numbers", Int. J. of Mod. Phys. B 20, Nos. 11-13, p. 1893-1916 (2006). Copyright 2006 by World Scientific Publishing Co. Pte. Ltd.. 
phase factors in the sum contribute with unity and thus the resulting signal value $\left|\mathcal{A}_{N}^{(\ell-1)}\right|$ is equal to one. In contrast, for non-factor arguments we find a suppressed value of the signal.

The Gauss reciprocity relation[Han80, Mat03]

$$
\sum_{m=0}^{a-1} \exp \left[i \pi m^{2} \frac{b}{a}\right]=\sqrt{\frac{a i}{b}} \sum_{m=0}^{b-1} \exp \left[-i \pi m^{2} \frac{a}{b}\right]
$$

establishes a connection to the previously discussed type of Gauss sum $\mathcal{S}_{N}$.

When we identify $a=\ell$ and $b=2 N$ we find the alternative representation

$$
\mathcal{A}_{N}^{(\ell-1)}(\ell)=\frac{1}{\ell} \sqrt{\frac{i \ell}{2 N}} \sum_{m=0}^{2 N-1} \exp \left[-2 \pi i m^{2} \frac{\ell}{4 N}\right] .
$$

Using the fact that this Gauss sum is periodic with period $4 N$ we find

$$
\begin{aligned}
\mathcal{A}_{N}^{(\ell-1)}(\ell) & =\frac{1}{2 \ell} \sqrt{\frac{i \ell}{2 N}} \sum_{m=0}^{4 N-1} \exp \left[-2 \pi i m^{2} \frac{\ell}{4 N}\right] \\
& \equiv \frac{\mathrm{e}^{i \frac{\pi}{4}}}{2} \frac{1}{\sqrt{2 \ell N}} G(\ell, 4 N) .
\end{aligned}
$$

The advantage of $G(\ell, 4 N)$ relies on the fact that there exist analytical expressions for calculating the signal value $\left|\mathcal{A}_{N}^{(\ell-1)}(\ell)\right|$ depending on which of the four classes $\mathcal{M}_{k}$, Eq. (2.28), the argument $\ell$ belongs to. In Appendix $\mathrm{D}$ we derive analytical expressions for the signal value $\left|\mathcal{A}_{N}^{(\ell-1)}\right|$ for any type of argument $\ell$. Here we provide a brief summary of these results.

- All arguments $\ell$ corresponding to a divisor $p$ of $N$ are characterized by the maximum value

$$
\left|\mathcal{A}_{N}^{(\ell-1)}(p)\right|=1
$$

in the factorization interference pattern, Eq. (D.3). Here the term divisor covers both prime factors and also products of prime factors.

- For all arguments $\ell \in \mathcal{M}_{2}$ the value of the signal

$$
\left|\mathcal{A}_{N}^{(\ell-1)}(\ell)\right|=0
$$

vanishes, Eq. (D.4).

- If the argument $\ell$ and the number $N$ to be factorized share no common factors we find

$$
\left|\mathcal{A}_{N}^{(\ell-1)}(\ell)\right|=\sqrt{\frac{1}{\ell}} \quad \text { or } \quad\left|\mathcal{A}_{N}^{(\ell-1)}(4 \ell)\right|=\sqrt{\frac{2}{\ell}}
$$

from Eq. (D.5) and Eq. (D.6). 
- If the argument $\ell$ and the number $N$ to be factorized share a common factor $p$ we find

$$
\left|\mathcal{A}_{N}^{(\ell-1)}(j p)\right|=\frac{1}{\sqrt{j}} \quad \text { or } \quad\left|\mathcal{A}_{N}^{(\ell-1)}(4 j p)\right|=\frac{1}{\sqrt{2 j}}
$$

from Eq. (D.7) and Eq. (D.8). Here $j$ is an integer.

In Figure 2.6 we show the factorization interference pattern arising from the complete Gauss sum, Eq. (2.36), for the number $N=1911$. This type of Gauss sum allows for an excellent discrimination of factors from non-factors. Most data-points are located on one of the four curves given by Eqs. (D.5)-(D.6). We also find that datapoints which are not situated on either of those curves carry information on one of the factors of $N$. This fact is in line with our analytical results of Eqs. (D.7)-(D.8).

Despite these convincing analytical properties the complete Gauss sum is disadvantageous when we consider the total number of terms which have to be summed in order to obtain a complete factorization interference pattern. According to Eq. (2.36) the upper bound in the summation depends on the argument $\ell$. In the context of factorization we are testing integer arguments up to $\sqrt{N}$. Hence, for a complete factorization interference pattern we have to add in total

$$
\sum_{\ell=1}^{\sqrt{N}} \ell=\frac{\sqrt{N}(\sqrt{N}+1)}{2} \approx \frac{N}{2}
$$

terms.

In the context of an experimental realization we consider the number of terms contributing to the Gauss sum as a resource for factorization. In the following we analyze the properties of Gauss sums with a truncated summation range.

\subsubsection{Factorization based on truncated Gauss sums}

Aim of this section is to analyze the properties of the truncated Gauss sum

$$
\mathcal{A}_{N}^{(M)}(\ell)=\frac{1}{M+1} \sum_{m=0}^{M} \exp \left(2 \pi i m^{2} \frac{N}{\ell}\right),
$$

in contrast to the complete Gauss sum of Eq. (2.36). This analysis is motivated by the idea to treat the number of terms in the Gauss sum as a resource for this factorization scheme. When we fix the upper bound in the summation of the truncated Gauss sum we would require the total number of

$$
\sum_{\ell=1}^{\sqrt{N}} M=\sqrt{N} M
$$




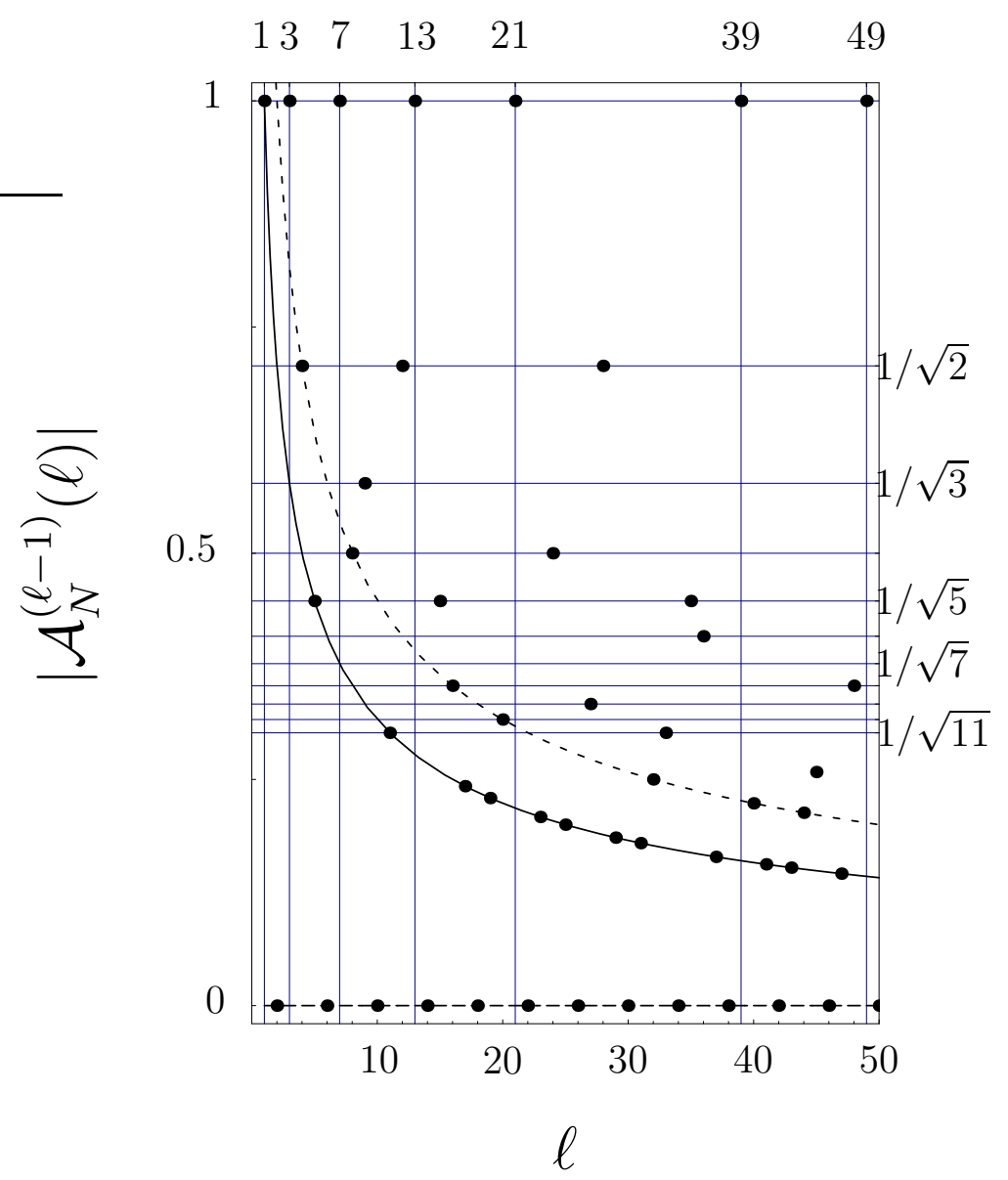

Figure 2.6: Factorization of $N=1911=3 \cdot 7^{2} \cdot 13$.

We depict the signal $\left|\mathcal{A}_{N}^{(\ell-1)}(\ell)\right|$ for integer arguments $\ell$. The positions of the prime factors (and products of them) are indicated by vertical lines. At the prime factors of $N$ the signal displays a maximum whereas the value of the signal is suppressed at non-factors. For each argument we provide an analytical expression for the absolute value of the signal $\left|\mathcal{A}_{N}^{(\ell-1)}\right|$. It is remarkable that the signal vanishes for all arguments $\ell$ out of the class $\mathcal{M}_{2}$ (dashed straight line). If the argument $\ell$ and the number $N$ to be factorized have no factors in common we find $\left|\mathcal{A}_{N}^{(\ell-1)}\right|=\sqrt{1 / \ell}$ (black curve). For arguments $\ell$ belonging to $\mathcal{M}_{0}$ that is $\ell=4 j$ with $j$ being no factor of $N$, we find $\left|\mathcal{A}_{N}^{(\ell-1)}\right|=\sqrt{2 / \ell}$ (dashed curve). Data-points which are not situated on either of those three curves indicate that the corresponding argument $\ell$ shares a common factor with $N($ e.g. $\ell=12, \ldots)$. 

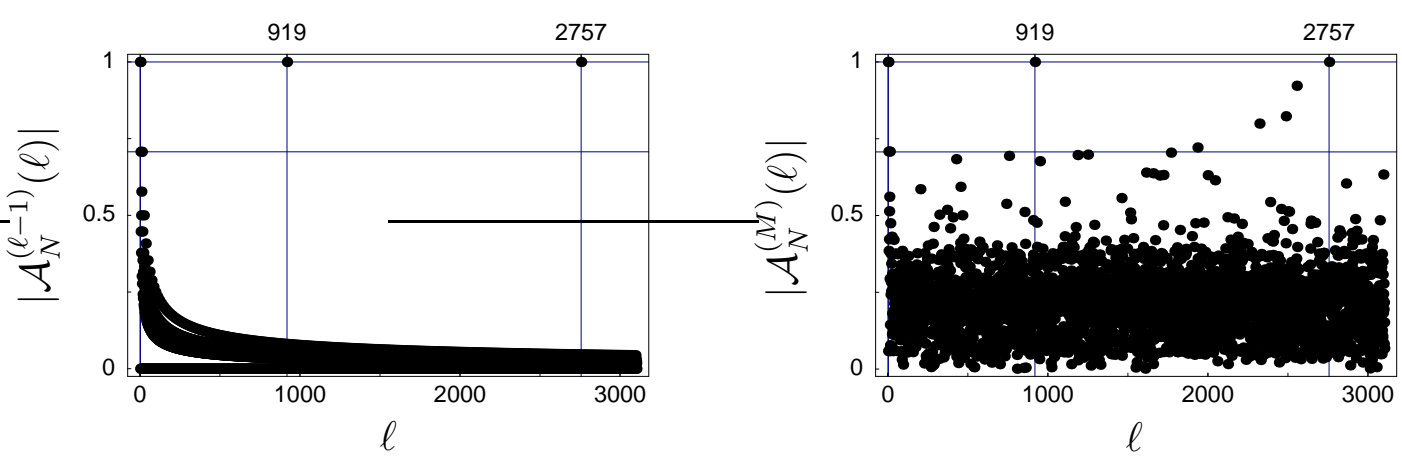

Figure 2.7: Effects of truncating the summation range in the Gauss sum for $N=$ $9624687=3 \cdot 919 \cdot 3491$.

We show the associated factorization interference patterns of the complete Gauss sum $\mathcal{A}_{N}^{(\ell-1)}(\ell)$, Eq. (2.36) (left), and the truncated Gauss sum, Eq. (2.41), with $M=\ln N=$ 16 terms. Vertical lines denote prime factors and product of prime factors. The complete Gauss sum reveals an impressive contrast due to a suppressed signal value at all nonfactors. However, also the truncated Gauss sum originating from a small number of terms allows to discriminate factors from non-factors.

terms for obtaining a complete factorization interference pattern.

In order to illustrate the effect of truncating the Gauss sum we compare in Figure 2.7 the factorization interference patterns for the complete Gauss sum $\mathcal{A}_{N}^{(\ell-1)}(\ell)$, Eq. (2.36) and for the truncated Gauss sum $\mathcal{A}_{N}^{(M)}(\ell)$, Eq. (2.41). In a first guess we chose the truncation parameter $M$ to depend logarithmically on the number to be factorized. It is remarkable that already the small number $M=16$ of terms in the truncated Gauss sum is sufficient to reveal the factors of the seven-digit number $N=9624687$.

\section{Natural threshold}

For arguments corresponding to factors of $N$ each term in the truncated Gauss sum is unity and hence the value of the normalized Gauss sum of Eq. (2.41) is also unity. Now we consider arguments $\ell=4 p$ which can be written as four times a factor $p$ of $N$.

For such arguments we find that the ratio $N / \ell$ can be decomposed

$$
\frac{N}{\ell} \equiv \text { integer } \pm \frac{1}{4}
$$

into an integer part and a fractional part.

Using this identity the exponential in Eq. (2.41) reduces and we find

$$
\mathcal{A}_{N}^{(M)}(4 p)=\frac{1}{M+1} \sum_{m=0}^{M}( \pm i)^{m^{2}} .
$$


Depending on the parity of the upper summation bound $M$ we find

$$
\mathcal{A}_{N}^{(M)}(4 p)=\frac{1}{2}\left\{\begin{array}{ccc}
\frac{1}{(1+1 / M)}(1+2 / M+i) & \text { for } & M \text { even } \\
(1+i) & \text { for } & M \text { odd }
\end{array}\right.
$$

In the context of factorization we are interested in the absolute value of $\mathcal{A}_{N}^{(M)}$. For odd values of $M$ the modulus takes on the value

$$
\left|\mathcal{A}_{N}^{(M)}(4 p)\right| \equiv \frac{1}{\sqrt{2}}
$$

and for even $M$ we find that the sum converges to the same threshold value

$$
\left|\mathcal{A}_{N}^{(M)}(4 p)\right| \rightarrow \frac{1}{\sqrt{2}}
$$

as we increase $M$

Thus in addition to arguments corresponding to factors of $N$ we find another class of arguments which independently of the choice of the truncation parameter $M$ result in signal values of $1 / \sqrt{2}$.

Hence we can speak of a natural gap between arguments corresponding to factors of $N$ with $\left|\mathcal{A}_{N}^{(M)}\right|=1$ and arguments which can be written as $\ell \equiv 4 p$ with associated signal value $\left|\mathcal{A}_{N}^{(M)}\right|=1 / \sqrt{2}$.

\section{Problem of ghost factors}

When we reconsider Figure 2.7, we observe a number of datapoints with signal values $1 / \sqrt{2}<\left|\mathcal{A}_{N}^{(M)}\right|<1$ for the truncated Gauss sum, for example at arguments $\ell=2487$ and 2555 . In the interpretation of the factorization interference pattern such datapoints could be misinterpreted as factors. In the following we analyze arguments for which the signal takes on such exceptionally high values.

For arguments $\ell$, for which the ratio $N / \ell$ is close to an integer value, the factorization interference pattern shows a maximum value close to unity, even though $\ell$ is not a factor of $N$. We have coined the expression "ghost factor" for such arguments. Having identified a datapoint with large signal value the corresponding argument has to be tested on a classical computer whether it divides $N$ without residue.

In the context of factorization the truncated Gauss sum can be viewed to serve as a tool to reduce the number of candidate factors (which would have to be tested for division).

Counting ghost factors

For the factorization interference pattern shown on the right in Figure 2.7 we have chosen a logarithmic dependence $M \sim \ln N$ of the truncation parameter 


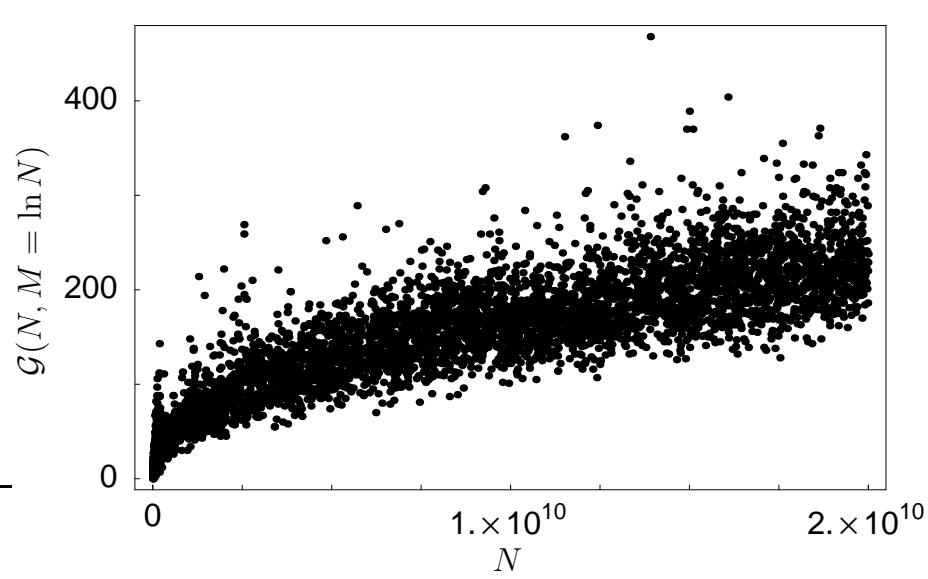

Figure 2.8: Ghost factor counting function $\mathcal{G}(N, M)$ calculated for 6333 random odd numbers $N$ out of the interval $\left[1,2 \cdot 10^{10}\right]$ and a logarithmic dependence of the upper bound $M \sim \ln N$ in the summation of $\mathcal{A}_{N}^{(M)}$. This plot indicates that $\mathcal{G}(N, \ln N)$ grows faster than the logarithm of $N$.

$M$ on the number $N$ to be factorized. But does this choice really allow for sufficient contrast in the factorization interference pattern?

To answer this question we introduce the ghost factor counting function

$$
\mathcal{G}(N, M) \equiv\left\{\text { number of datapoints with } \frac{1}{\sqrt{2}}<\left|\mathcal{A}_{N}^{(M)}(\ell)\right|<1\right\}
$$

which yields the number of data-points with critical values of the signal in the factorization interference pattern for a given $N$ and a chosen upper bound $M$.

In order to study the behavior of the ghost factor counting function $\mathcal{G}(N, M)$ on a broad range of numbers $N$ we show in Figure $2.8 \mathcal{G}(N, M=\ln N)$ for 6333 odd random numbers $N$ out of the interval $\left[1,2 \cdot 10^{10}\right]$. Here we choose the truncation parameter to depend logarithmically $M \sim \ln N$ on $N$. This result shows that the number of ghost factors $\mathcal{G}(N, M)$ for $M \sim \ln N$ grows faster than the logarithm of $N$. When we compare this scaling of the number of ghost factors to the maximal number $\sim \ln N$ of actual factors we conclude that our first guess for $M$, the logarithmic choice, is not optimal.

\section{Ghost factors in the complex plane}

An alternative illustration of the emergence of the value of the unnormalized Gauss sum $(M+1) \mathcal{A}_{N}^{(M)}$ in the complex plane is also well suited to shed light on the ghost factor phenomenon. We note that M. V. Berry has employed this representation of Gauss sums in the context of curlicues [Ber88b, Ber88a].

Here we analyze trajectories in the complex plane which is spanned by the real part of the unnormalized Gauss sum $(M+1) \operatorname{Re} \mathcal{A}_{N}^{(M)}(\ell)$ and the imaginary 
part $(M+1) \operatorname{Im} \mathcal{A}_{N}^{(M)}(\ell)$. We illustrate how the final value of the unnormalized Gauss sum $(M+1) \mathcal{A}_{N}^{(M)}$ in Eq. (2.41) constitutes as we increase the number $M$ of terms in the sum.

In Figure 2.9 we present some Gauss sum trajectories for various arguments $\ell$. For factors of $N$ the corresponding trajectory moves outward on the real axis (without ever acquiring any imaginary admixtures). For non-factors the associated trajectories are confined to a relatively small area in this complex plane so that the value of the signal is well suppressed. This suppression is due to the fact that the trajectories are reminiscent to random walks. For ghost factor arguments the associated trajectories move also outward in the complex plane. However, such trajectories are accompanied by an accrual of non-zero imaginary parts. This fact provides a criterion to distinguish ghost factors from actual factors.

In addition, this representation provides a qualitative hint for the choice of the optimal upper bound $M$ for the summation of the Gauss sum. For small values of $M$ it is hard to discriminate factors from ghost factors because the latter are characterized by small imaginary parts. For a ghost factor the accumulated imaginary part becomes only significant as we increase $M$.

\section{Optimal choice for the truncation parameter $M$}

Here we analyze the dependence of the signal value $\left|\mathcal{A}_{N}^{(M)}\right|$ on the upper summation limit $M$ in more detail. For this purpose we study how the signal value associated with a ghost factor emerges as a function of $M$. In Figure 2.10 we study the signal $\left|\mathcal{A}_{N}^{(M)}\right|$ for $N=9624687$ and the ghost factor argument $\ell=2555$ when we increase the number of terms in the Gauss sum. For small $M$ the value of the signal hardly deviates from unity. As we increase $M$ further we find that the signal value $\left|\mathcal{A}_{N}^{(M)}\right|$ can indeed be suppressed to non-critical values below the natural threshold $1 / \sqrt{2}$.

For this purpose we analyze the phases

$$
\phi_{m} \equiv 2 \pi m^{2} \frac{N}{\ell}
$$

for critical arguments $\ell$ resulting in ghost factors. In any case the ratio $N / \ell$ can be decomposed

$$
\frac{N}{\ell}=\text { integer }+\tau
$$

into an integer part and into a fractional part $\tau$ with $|\tau|<1 / 2$. In the context of factorization we restrict our analysis to arguments $\ell$ belonging to the interval $[1, \sqrt{N}]$. Hence, the smallest possible fractional part is given by

$$
\tau_{\min } \sim \frac{1}{\sqrt{N}}
$$




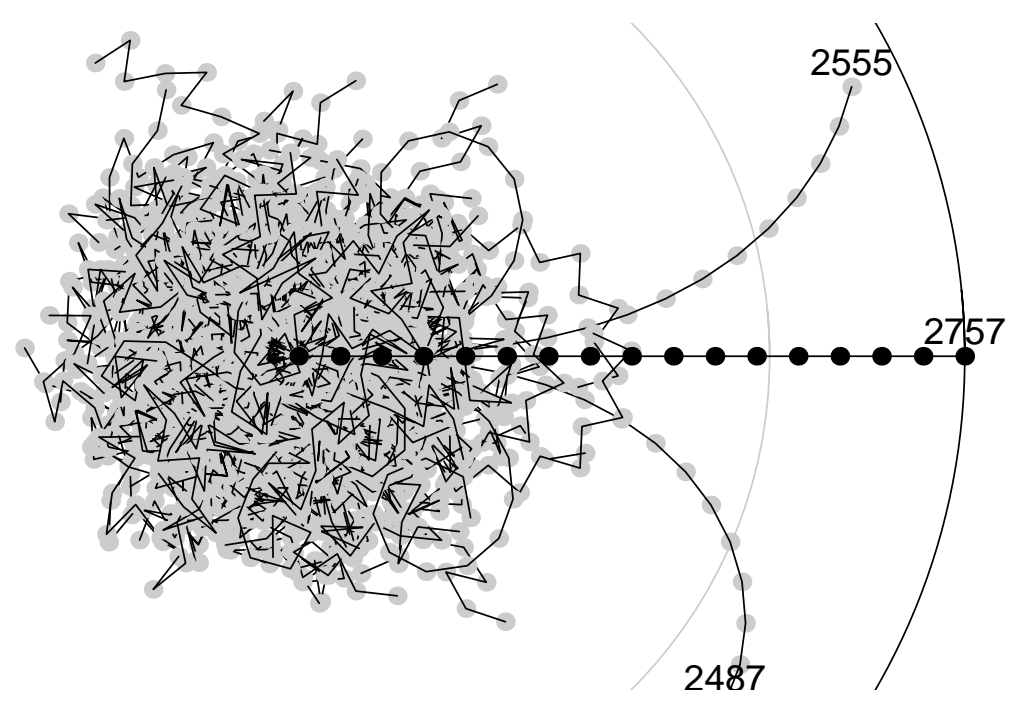

Figure 2.9: Trajectories for factors and ghost factors in the complex plane.

We consider the unnormalized Gauss sum $(M+1) \mathcal{A}_{N}^{(M)}$ for the number $N=9624687$ and an increasing number of terms: $M=0 \ldots 16$. The complex plane is spanned by the real part $(M+1) \operatorname{Re} \mathcal{A}_{N}^{(M)}(\ell)$ and the imaginary part $(M+1) \operatorname{Im} \mathcal{A}_{N}^{(M)}(\ell)$. This alternative representation of the Gauss sum allows us to illustrate the characteristic trajectories of the signal value $\mathcal{A}_{N}^{(M)}$ for both factors and non-factors of $N$. The circle segment with radius $(M+1)$ denotes the maximal value of the unnormalized Gauss sum. The gray circle segment with radius $(M+1) / \sqrt{2}$ depicts the natural threshold between well-suppressed data points and ghost factors. Factors of $N$ move outward on the real axis as a function of the upper bound in the Gauss sum (e.g. $\ell=2757$, denoted by black dots) without aquiring any imaginary part. For most non-factors the trajectories are reminiscent to random walk such that they take place in a well-confined area in the complex plane. However, for some arguments $\ell$ the trajectories move also outward while acquiring non-zero imaginary parts, for example $\ell=2487$ and 2555 . We call such arguments "ghost factors". 
With the decomposition of Eq. (2.50) the Gauss sum Eq. (2.41) simplifies

$$
\mathcal{A}_{N}^{(M)}(\ell)=\frac{1}{M+1} \sum_{m=0}^{M} \exp \left(2 \pi i m^{2} \tau\right)
$$

since each phase factor of the type $\exp \left(2 \pi i \mathrm{~m}^{2}\right.$. integer $)$ yields a factor of unity.

When we analyze the fraction $N / \ell$ for ghost factors we find that the corresponding arguments are characterized by small values of $\tau \rightarrow 0$. The associated trajectories in Figure 2.9 are confined to a sector in the right half plane with positive real part $(M+1) \operatorname{Re} \mathcal{A}_{N}^{(M)}>0$ and small imaginary parts $(M+1)\left|\operatorname{Im} \mathcal{A}_{N}^{(M)}\right|$.

Since we are interested in an approximation for small values of $\tau$ we estimate that the difference of consecutive terms in the truncated Gauss sum is small. Therefore we replace the summation in the Gauss sum by an integral

$$
\mathcal{A}_{N}^{(M)}(\ell) \approx \frac{1}{2 M \sqrt{\tau}} \int_{0}^{2 M \sqrt{\tau}} d u \exp \left(i \frac{\pi}{2} u^{2}\right)=\frac{F(2 M \sqrt{\tau})}{2 M \sqrt{\tau}}
$$

where

$$
F(x)=\int_{0}^{x} d u \exp \left(i \frac{\pi}{2} u^{2}\right)
$$

denotes the Fresnel Integral[Abr72].

Here we have modified the prefactor $(1 / M$ instead of $1 /(M+1))$ in order to guarantee that our approximation of the Gauss sum yields the same result

$$
\lim _{M \rightarrow 0} \frac{F(2 M \sqrt{\tau})}{2 M \sqrt{\tau}}=1=\mathcal{A}_{N}^{(M=0)}(\ell)
$$

in the limit of $M \rightarrow 0$. It is remarkable that the upper summation index $M$ and the fractional part $\tau$ always appear in the combination $M \sqrt{\tau}$.

Let us point out the range of applicability of the approximation Eq. (2.53). According to [Ber88b] the phase difference

$$
\left|\phi_{m+1}-\phi_{m}\right|=2 \pi(2 m+1) \tau
$$

of two successive terms in the sum Eq. (2.41) should at most be of the order of $\pi$. Together with the fact that the maximal phase difference appears for $m=M$ we arrive at the inequality

$$
2 \tau(2 M+1)<1 .
$$

Recalling the smallest fractional part from Eq. (2.51) we arrive at the constraint

$$
M_{c} \approx \frac{1}{4} \sqrt{N}
$$


on the maximal value $M_{c}$ of the truncation parameter for a given $N$. Thus $M_{c}$ serves as an upper bound for the validity of the Fresnel approximation, Eq. (2.53).

In Figure 2.10 we show the absolute value at a ghost factor argument for both the discrete truncated Gauss sum, Eq. (2.41), and the Fresnel integral $F(2 M \sqrt{\tau}) /(2 M \sqrt{\tau})$. We find that the continuous approximation by means of the Fresnel integral impressively models the discrete results of the Gauss sum.

We are now looking for the ideal truncation parameter $M_{0}$ so that for a given minimal fractional part $\tau_{\min }$ the absolute value of the integral Eq. (2.53) is smaller than $1 / \sqrt{2}$. The point

$$
\alpha=2 M_{0} \sqrt{\tau_{\min }}
$$

where the Fresnel approximation, Eq. (2.53), attains the threshold of $1 / \sqrt{2}$ is determined numerically as $\alpha \approx 1.318$. When we recall the minimum value of $\tau_{\min }$, Eq. (2.51), we eventually arrive at

$$
M_{0} \sim \sqrt[4]{N}
$$

which is well within the validity of the Fresnel approximation since $M_{0}<M_{c}$.

In Figure 2.11 we compare the factorization interference patterns for two choices of the truncation parameter. On the left we choose $M=\ln N$ according to our initial guess whereas on the right we choose the optimal truncation parameter $M_{0}$ according to Eq. (2.60). The optimally truncated Gauss sum leads to a full suppression of all non-factors below the natural threshold.

However, this dependence of the optimal upper bound $M_{0}$ is not favorable since it scales like a power law as a function of $N$. In Ref. [Š06] we show that this dependence cannot be changed.

For a complete factorization interference pattern in the optimal case we require the total number of terms

$$
\sum_{\ell=1}^{\sqrt{N}} M_{0}=\sqrt{N} \sqrt[4]{N}=N^{3 / 4},
$$

which is still advantageous over factorization based on the complete Gauss sum $\mathcal{A}_{N}^{(\ell-1)}$.

\subsubsection{Complexity of the factorization scheme}

In computer science problems are classified depending on their complexity. Here the central question asks for the scaling of the required time or resources for a given problem as a function of the input.

We address this question for the present factorization scheme. For this purpose we treat the number of digits $L=\ln N$ of $N$ as the input. 


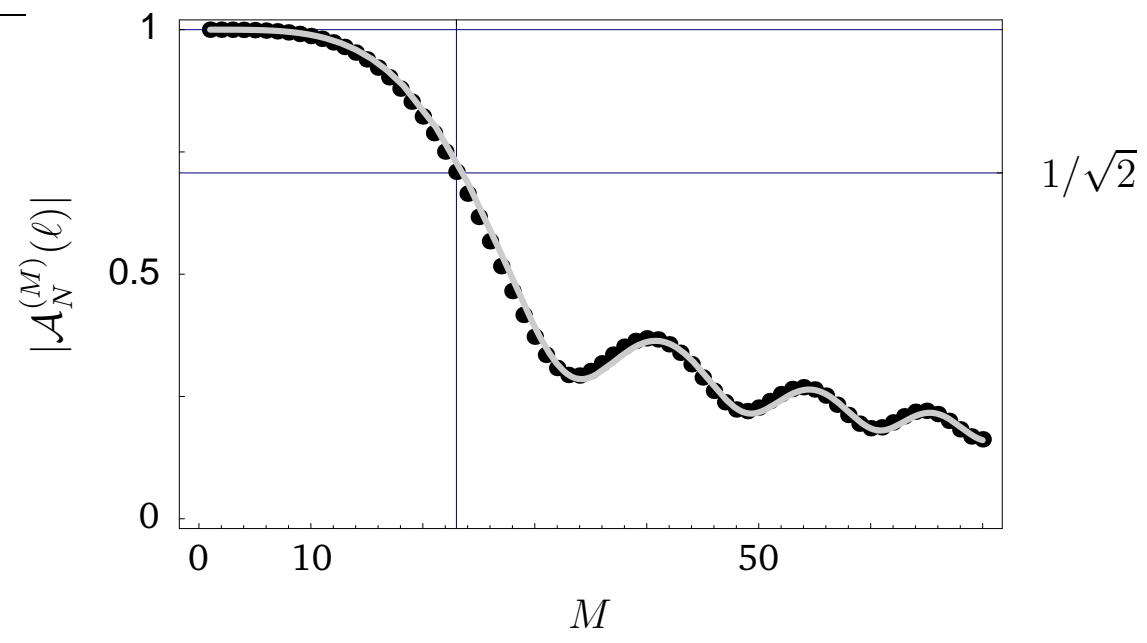

Figure 2.10: Signal at the ghost factor $\ell=2555$.

As before we consider the number to be factorized $N=9624687$ and vary the number of terms in the Gauss sum for the fixed argument $\ell=2555$. Here the fractional part of $N / \ell$ is given by $\tau=2 / 2555$. We depict the absolute value $\left|\mathcal{A}_{N}^{(M)}(\ell)\right|$ as a function of the number $M$ of terms contributing to the Gauss sum Eq. (2.41). The gray line shows the Fresnel approximation, Eq. (2.53), for the Gauss sum.
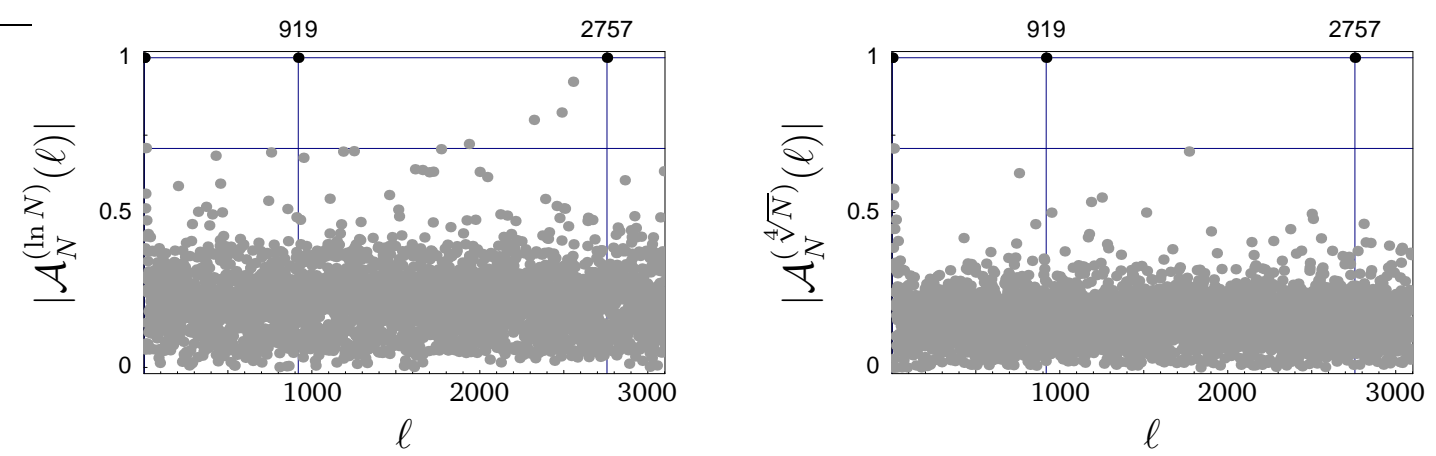

Figure 2.11: Optimal truncation of the Gauss sum for $N=9624687=3 \cdot 919 \cdot 3491$.

We compare the associated factorization interference patterns of the Gauss sum $\mathcal{A}_{N}^{(M)}(\ell)$, Eq. (2.41), for two choices of the truncation parameter $M$. On the left the truncation parameter is chosen according to $M=\ln N=16$. On the right we have truncated the Gauss sum after $M_{0}=29$ terms. Indeed, for the optimal choice of the truncation parameter we suppress the signal value for all non-factors below the natural threshold of $1 / \sqrt{2}$. 
Testing integer arguments

For the factorization of a number $N$ our scheme requires at most $\sqrt{N}$ tests of the signal value $\left|\mathcal{A}_{N}^{(M)}\right|$ at all integer arguments. We estimate the required resources as

$$
\sqrt{N}=\exp \left(\frac{L}{2}\right)
$$

where $L \equiv \ln N$ is the number of digits of $N$. Although our scheme scales exponentially as a function of $L$ we profit from the small number of terms $M$ necessary to distinguish factors from non-factors.

\section{Testing only prime arguments}

We can reduce the number of tests by restricting the tests of the signal value only to prime arguments. For this choice the number of tests reduces to

$$
\pi(\sqrt{N}) \sim \frac{\sqrt{N}}{\ln \sqrt{N}}=\frac{2}{L} \exp \left(\frac{L}{2}\right)
$$

where $\pi(\sqrt{N})$ is the number of primes smaller than $\sqrt{N}$. Moreover, we have used the approximation

$$
\pi(x) \approx \frac{x}{\ln x}
$$

for the prime counting function.

Thus the number of tests still scales exponentially as a function of $L$. However, the pre-factor $2 / L$ weakens this scaling law in comparison to the previous scheme. Another advantage of sampling at prime arguments consists in the fact that maximum signal values directly indicate prime factors of $N$.

For this scheme in a first step we have to test whether a given integer argument is a prime number or not. For smaller arguments there are prime number tables whereas for larger arguments we first have to check whether a given argument $\ell$ is prime. There is a known algorithm for solving this problem on a classical computer in polynomial time [Agr04].

\subsection{Discussion}

In this chapter we have presented various schemes to factorize numbers on the basis of two types of Gauss sums:

(i) $\mathcal{S}_{N}$ where $N$ appears in the denominator of the phase factor, and

(ii) $\mathcal{A}_{N}^{(M)}$ where the roles of $N$ and $\ell$ are interchanged and the summation range is truncated. 
For the first type we propose two kinds of factorization schemes which base on continuous and discrete sampling of $\mathcal{S}_{N}$. The continuous version of this factorization scheme features a remarkable scaling property: A single realization of the Gauss sum for the number $N$ can be used to factor another number $\widetilde{N}$. The discrete version of this factorization scheme is restricted to measurements of the signal at integer arguments.

The Gauss reciprocity relation establishes the link between both types of Gauss sums. For $\mathcal{A}_{N}^{(M)}$ we consider only the discrete sampling of the signal and study two cases regarding the summation range. For the complete Gauss sum the summation covers a whole period, whereas the truncated version has the advantage that already a limited number of terms enables us to distinguish factors from non-factors.

The results of this chapter provide the motivation for the following chapters 3 and 4 where we study three physical systems which involve Gauss sums in a measurable quantity. In chapter 5 we demonstrate the factorization property for each of the physical systems. 


\section{CHAPTER 3}

\section{Gauss sums arising from a chirped two-photon transition}

In the following, we show that Gauss sums naturally emerge in the physical system of a two-photon transition in a specific multistate ladder system when driven by a chirped laser pulse.

This chapter is organized as follows: In section 3.1 we provide an introduction into chirped laser pulses and their applications in modern physics. In section 3.2 we first investigate the excitation in the simple three-state ladder system driven by a weak chirped laser pulse and develop a phase space argument for the occurrence of quadratic phase factors.

In section 3.3 we apply these results to a specific ladder system and show that the excitation probability amplitude is of the form of a Gauss sum. We devote section 3.4 to a discussion of standard quantum optical systems which may serve as candidates for realizing Gauss sums in an experiment.

\subsection{Chirped laser pulses}

Chirped laser pulses are characterized by a non-linear phase dependence

$$
\phi(\omega)=\phi_{0}+\phi^{\prime}\left(\omega-\omega_{0}\right)++\frac{\phi^{\prime \prime}}{2}\left(\omega-\omega_{0}\right)^{2}+\ldots
$$

In the following we focus on pulses with quadratic non-linearity $\phi^{\prime \prime} \neq 0$ which are characterized by a linear variation of the frequency. 


\subsubsection{Description of chirped pulses}

The electric field of the chirped laser pulse with complex-valued pulse shape $f(t)$ is given by

$$
E_{\mathrm{c}}(t)=\mathcal{E}_{0}\left[e^{-i \omega_{0} t} f(t)+\text { c.c. }\right],
$$

with amplitude $\mathcal{E}_{0}$ and carrier frequency $\omega_{0}$.

The pulse shape

$$
f(t)=f_{0} \exp \left(-\alpha_{r} t^{2}\right) \exp \left(-i \alpha_{i} t^{2}\right)=f_{0} \exp \left[-\frac{1}{2}\left(\Delta \omega f_{0}\right)^{2} t^{2}\right]
$$

contains both a real-valued Gaussian of width

$$
\alpha_{r} \equiv \frac{\Delta \omega^{2}}{2} \frac{1}{1+a^{2}}
$$

and an imaginary Gaussian of width

$$
\alpha_{i} \equiv \frac{\Delta \omega^{2}}{2} \frac{a}{1+a^{2}}=a \alpha_{r}
$$

Here we have expressed the dependence on the chirp $\phi^{\prime \prime}$ by introducing the dimensionless quadratic phase

$$
a \equiv \Delta \omega^{2} \phi^{\prime \prime}
$$

where $\Delta \omega$ denotes the bandwidth. In Eq. (3.3) we have also made use of the complex-valued quantity

$$
\frac{1}{2}\left(\Delta \omega f_{0}\right)^{2} \equiv \alpha_{r}+i \alpha_{i}
$$

which depends on the widths $\alpha_{r}$ and $\alpha_{i}$ defined in Eq. (3.4) and Eq. (3.5).

The imaginary Gaussian in the pulse envelope is responsible that the "instantaneous frequency"

$$
\omega(t)=\omega_{0}+2 \alpha_{i} t=\omega_{0}+\Delta \omega^{2} \frac{a}{1+a^{2}} t
$$

varies linearly as the laser pulse propagates in time. A change in sign of $\phi^{\prime \prime}$ corresponds to a reversed order of the frequency sweep.

We note that a pulse with vanishing second order dispersion $\left(\phi^{\prime \prime}=0\right)$ is a Fourier-transform (FT) limited pulse, i.e. a pulse with the shortest possible duration for a given bandwidth. 


\subsubsection{Applications of chirped pulses}

Among others chirped pulses serve as a powerful tool for coherent control of quantum systems [Sha00]. This objective has attracted a lot of interest during the last decades [Rab00]. The primary goal is to coherently steer quantum systems such as atoms and molecules to a desired final state by tailoring the interaction with light.

In the following we provide an overview of some of the widespread applications of chirped pulses which rely on both strong and weak fields.

\section{Strong field regime}

In the strong-field regime adiabatic passage has proven to serve as an excellent tool for efficient population transfer in various systems [Vit01]. The most prominent example of population transfer by means of adiabatic passage is probably the STIRAP-method (Stimulated Raman Adiabatic Passage) [Ber98] in a $\Lambda$-configuration. Here, population is transferred with high efficiency by two strong pulses arriving in the counter-intuitive order [Ore84]. Thus, transient population of the intermediate state can be suppressed to a great extent. The most important advantage of STIRAP consists in its robustness to variations in the pulse parameters, which is in contrast to standard Rabi cycling [Sho90].

When we consider molecular ladders [Che90], a similar excitation can be accomplished with a single chirped pulse which features an intuitive frequency sweep, that is, the frequency of the pulse decreases with time while the separation of states in the ladder decreases as higher lying states are excited.

Two-photon absorption (TPA) in the atomic three-state ladder system $5 \mathrm{~s} \leftrightarrow$ $5 p \leftrightarrow 5 d$ of $\mathrm{Rb}$ was studied for the case of chirped pulses with both intuitive and counter-intuitive frequency sweeps [Bro92, Maa99]. When the frequency sweep follows the ladder spacing, complete population transfer to the upper state is achieved with the side effect that the intermediate state is substantially populated during a transient stage. A pulse with a counter-intuitive frequency sweep still leads to $100 \%$ transfer but at the same time also suppresses transient population of the intermediate state.

Adiabatic population transfer using strong field chirped pulses can be performed by the SCRAP-method (Stark Chirped Rapid Adiabatic Passage) [Ran05].

Adiabatic passage induced by a chirped laser pulse is also an ideal tool for state preparation in single trapped ions [Wun06]. Due to the robustness of this scheme applications in quantum computing offer themselves. 
Gauss sums arising from a chirped two-photon transition

Weak-field regime

In the low-field regime the population transfer is determined by the coherent transient (CT), which is the integral over the electric field associated with the laser pulse. In this regime the transfer is non-adiabatic and only very little population can be transferred.

When an atomic three-state ladder is driven by a weak chirped pulse [Bal94], various excitation mechanisms can be identified and the transferred population arises due to interference of different excitation paths, each of which is characterized by a value of the phase. As a consequence, the excited state population oscillates as a function of the frequency sweep.

In the context of weak-field excitation induced by an ultra-short chirped laser pulse in a two-level system [Zam01] coherent transients could be observed directly in an experiment. Here, most of the population transfer occurs when the frequency is resonant with the atomic transition. After sweeping through resonance the excited state population oscillates due to interference of resonant and non-resonant contributions. In this case the CT has the form of a Fresnel integral and the excited state population follows the famous Cornu spiral [Sch01]. This remarkable property is at the heart of Ref. [Deg02] where an analog of a time domain Fresnel lense was realized using a chirped pulses.

Another intersting application of weak chirped pulses is the two-photon absorption in a ladder system which involves two intermediate states [Cha03]. Here interference of multiple paths in quantum ladder climbing leads to strongly contrasted oscillations in the excited state population.

Chirped pulses are also employed as a tool for quantum state measurement [Mon06]. In contrast to pump-probe approaches, which consist of two stages, here the quantum state is measured in real time during the interaction with the unknown pulse.

Next we show that weak field excitation in a multi-state ladder induced by a chirped pulse leads to a Gauss sum in the excitation probability amplitude of reaching the excited state. In an experiment this quantity is accessible by observing the fluorescence signal of the excited state.

\subsection{Chirped pulse excitation in a three-state ladder}

We consider a simple three-state ladder system driven by a weak chirped laser pulse and calculate the excitation probability amplitude in second order perturbation theory. Moreover, we show that this probability amplitude depends quadratically on the offset of the intermediate state and provide a phase space argument for the occurrence of quadratic phase factors. 


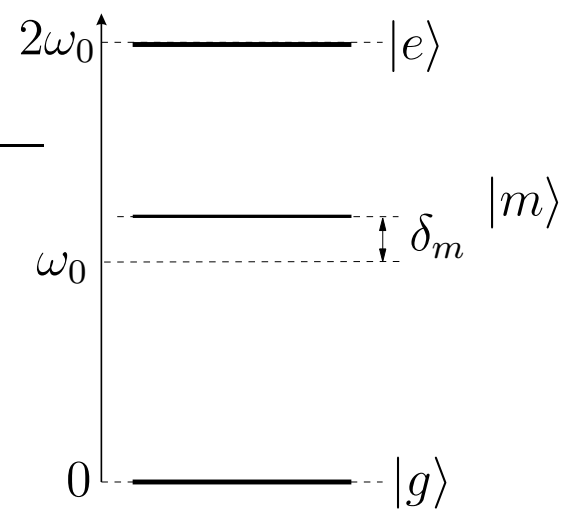

Figure 3.1: Three-state ladder system: The ground state $|g\rangle$ is connected by a two-photon transition to the excited state $|e\rangle$. In addition there is an intermediate state $|m\rangle$, which is displaced by the offset $\delta_{m}$ with respect to the central frequency $\omega_{0}=\omega_{g e} / 2$.

\subsubsection{Model}

The three-state ladder system consists of a ground state $|g\rangle$, an excited state $|e\rangle$ and an intermediate state $|m\rangle$, as depicted in Figure 3.1. The energy of the two-photon transition between ground and excited state is $2 \hbar \omega_{0}$-without loss of generality the ground state energy is set to zero. The intermediate state is shifted with respect to the central frequency $\omega_{0}$ by an offset $\delta_{m}$.

Hence, the total Hamiltonian is given by

$$
H=H_{0}+V_{\mathrm{S}}(t),
$$

where

$$
H_{0}=\hbar\left[\left(\omega_{0}+\delta_{m}\right)|m\rangle\left\langle m\left|+2 \omega_{0}\right| e\right\rangle\langle e|\right]
$$

is the free Hamiltonian of the ladder system and

$$
V_{\mathrm{S}}(t)=-d \cdot E_{\mathrm{c}}(t)
$$

describes the coupling of the dipole operator $d$ to the electric field of the chirped laser pulse $E_{\mathrm{c}}(t)$, Eq. (3.2). Here we have introduced the dipole operator

$$
d=\sum_{j=g, e}\left(\wp_{j m}|j\rangle\langle m|+\text { c.c. }\right)
$$

associated with the ladder system where the dipole matrix element $\wp_{j m}$ determines the coupling strength of the transition $|j\rangle \rightarrow|m\rangle$ with $|j\rangle=|g\rangle$ or $|e\rangle$.

\subsubsection{Time evolution}

In the following analysis, we consider the time evolution of the quantum state

$$
|\psi(t)\rangle=c_{g}(t)|g\rangle+c_{m}(t)|m\rangle+c_{e}^{(m)}(t)|e\rangle
$$


of the three-state ladder system. Here $c_{g}(t)$ and $c_{m}(t)$ denote the probability amplitude of finding the system in the ground or intermediate state respectively. The notation $c_{e}^{(m)}$ accounts for the fact that the promotion of the ladder system to the excited state $|e\rangle$ is accompanied by a transit of population through the intermediate state $|m\rangle$.

We assume that the temporal width of the chirped laser pulse is shorter compared to the characteristic time scale of decay processes in the three-state ladder.

Consequently, we may neglect spontaneous emission and thus the Schrödinger equation,

$$
i \hbar \frac{d}{d t}|\psi(t)\rangle=V(t)|\psi(t)\rangle
$$

is well suited to describe the time evolution of the quantum state Eq. (3.13) under the dipole coupling

$$
V(t)=-\sum_{j=g, e}\left[\wp_{j m} e^{i\left(\omega_{j}-\omega_{m}\right) t}|j\rangle\langle m|+\text { c.c. }\right] E(t)
$$

in interaction picture. Here $V(t)$ originates from $V_{\mathrm{S}}$, Eq. (3.11), via the transformation

$$
V(t)=\exp \left(\frac{i}{\hbar} H_{0} t\right) V_{\mathrm{S}}(t) \exp \left(-\frac{i}{\hbar} H_{0} t\right) .
$$

The interaction Hamiltonian $V(t)$ of Eq. (3.15) can be simplified further by performing the standard rotating wave approximation (RWA). The resulting approximate operator $V(t) \approx \mathcal{V}^{(m)}(t)$ can be written as the sum of two contributions

$$
\mathcal{V}^{(m)}(t)=\mathcal{V}_{g m}(t)+\mathcal{V}_{m e}(t)
$$

where

$$
\mathcal{V}_{g m}(t) \equiv-\hbar\left[\Omega_{m g} e^{i \delta_{m} t} f(t)|m\rangle\langle g|+\text { c.c. }\right]
$$

corresponds to the first excitation step $(|g\rangle \rightarrow|m\rangle)$ of the two-photon transition and

$$
\mathcal{V}_{m e}(t) \equiv-\hbar\left[\Omega_{e m} e^{-i \delta_{m} t} f(t)|e\rangle\langle m|+\text { c.c. }\right]
$$

accounts for the second step $(|m\rangle \rightarrow|e\rangle)$.

Moreover, we have defined the Rabi frequencies

$$
\Omega_{i j} \equiv \frac{\mathcal{E}_{0} \wp_{i j}}{\hbar}
$$

associated with each of the one-photon transitions in the three-state ladder system. 


\subsubsection{Perturbation theory in the weak field limit}

A formal integration of the Schrödinger equation (3.14) yields the time evolution operator

$$
U=\hat{T}\left[\exp \left(-\frac{i}{\hbar} \int_{-\infty}^{t} d t^{\prime} \mathcal{V}^{(m)}\left(t^{\prime}\right)\right)\right]
$$

where $\hat{T}$ establishes time ordering.

In the weak field limit we expand the time evolution operator

$$
U \approx U_{0}+U_{1}+U_{2}
$$

in second order perturbation theory. Assuming that our ladder system is initially prepared in the ground state $|g\rangle$ the excitation probability amplitude is given by

$$
c_{e}^{(m)}(t) \equiv\langle e|U| g\rangle .
$$

When we evaluate each contribution in the expansion Eq. (3.22), we find that the second order term

$$
c_{e}^{(m)}(t) \cong\left\langle e\left|U_{2}\right| g\right\rangle=-\frac{1}{2 \hbar^{2}} \int_{-\infty}^{t} d t^{\prime} \int_{-\infty}^{t^{\prime}} d t^{\prime \prime}\left\langle e\left|\mathcal{V}^{(m)}\left(t^{\prime}\right) \mathcal{V}^{(m)}\left(t^{\prime \prime}\right)\right| g\right\rangle
$$

is the first term in the series which yields a non-zero contribution to the excitation probability amplitude.

Using the explicit expressions for the interaction, Eqs. (3.17)-(3.19), we obtain

$$
c_{e}^{(m)}(t)=-\frac{\Omega_{e m} \Omega_{m g}}{2} \mathcal{I}_{m}
$$

where we have introduced the double integral

$$
\mathcal{I}_{m}=\int_{-\infty}^{t} d t^{\prime} e^{-i \delta_{m} t^{\prime}} f\left(t^{\prime}\right) \int_{-\infty}^{t^{\prime}} d t^{\prime \prime} e^{i \delta_{m}\left(t^{\prime \prime}\right)} f\left(t^{\prime \prime}\right)
$$

In the following we concentrate on the state of the ladder system after the laser pulse has passed, that is, we extend the upper integration limit in the first integral of $\mathcal{I}_{m}$ to infinity. When we introduce the new integration variables

$$
\bar{t} \equiv t^{\prime}+t^{\prime \prime} \quad \text { and } \quad t \equiv t^{\prime}-t^{\prime \prime}
$$

and rearrange the integration

$$
\int_{-\infty}^{\infty} d t^{\prime} \int_{-\infty}^{t^{\prime}} d t^{\prime \prime} \cdots=\frac{1}{2} \int_{-\infty}^{\infty} d \bar{t} \int_{0}^{\infty} d t \ldots
$$


we eventually arrive at

$$
c_{e}^{(m)}=-\frac{\Omega_{e m} \Omega_{m g}}{4} \int_{-\infty}^{\infty} d \bar{t} \int_{0}^{\infty} d t e^{-i \delta_{m} t} f\left(\frac{\bar{t}+t}{2}\right) f\left(\frac{\bar{t}-t}{2}\right) .
$$

Using the actual pulse shape $f(t)$ of the chirped laser pulse, Eq. (3.3), we arrive at

$$
c_{e}^{(m)}=-\frac{\Omega_{e m} \Omega_{m g}}{4} I_{1} \cdot I_{2}
$$

where the integrals are defined as

$$
I_{1} \equiv f_{0} \int_{-\infty}^{\infty} d \bar{t} e^{-\frac{1}{4}\left(\Delta \omega f_{0}\right)^{2} \bar{t}^{2}}=\frac{2 \sqrt{\pi}}{\Delta \omega}
$$

and

$$
I_{2} \equiv f_{0} \int_{0}^{\infty} d t e^{-i \delta_{m} t} e^{-\frac{1}{4}\left(\Delta \omega f_{0}\right)^{2} t^{2}}
$$

With the help of the complementary error function [Abr72]

$$
\operatorname{erfc}(z)=\frac{2}{\sqrt{\pi}} \int_{z}^{\infty} d u e^{-u^{2}} \quad \text { with } \quad \arg (z)<\frac{\pi}{4}
$$

we find

$$
I_{2}=\frac{\sqrt{\pi}}{\Delta \omega} \operatorname{erfc}\left(\frac{i}{f_{0}} \frac{\delta_{m}}{\Delta \omega}\right) \exp \left[-\left(\frac{1}{f_{0}} \frac{\delta_{m}}{\Delta \omega}\right)^{2}\right]
$$

and the excited state probability amplitude consequently reads

$$
c_{e}^{(m)}=-\frac{d_{m}}{2} \operatorname{erfc}\left(\frac{i}{f_{0}} \frac{\delta_{m}}{\Delta \omega}\right) \exp \left[-\left(\frac{1}{f_{0}} \frac{\delta_{m}}{\Delta \omega}\right)^{2}\right] .
$$

Here we have defined the prefactor

$$
d_{m} \equiv \pi \frac{\Omega_{e m} \Omega_{m g}}{\Delta \omega^{2}} .
$$

Making use of the definition of $f_{0}$, Eq. (3.7), we find for the excited state probability

$$
c_{e}^{(m)}=-\frac{d_{m}}{2} \operatorname{erfc}\left(i \frac{\delta_{m}}{\Delta \omega} \sqrt{1-i a}\right) \exp \left[-\left(\frac{\delta_{m}}{\Delta \omega}\right)^{2}\right] \exp \left[i\left(\frac{\delta_{m}}{\Delta \omega}\right)^{2} a\right] .
$$


We remark that this excitation probability amplitude already exhibits a phase factor which is quadratic in the offset $\delta_{m}$ of the intermediate state $|m\rangle$. In order to bring out this quadratic phase factor most clearly we introduce the weight factor

$$
w_{m} \equiv-\frac{d_{m}}{2} \operatorname{erfc}\left(i \frac{\delta_{m}}{\Delta \omega} \sqrt{1-i a}\right) \exp \left[-\left(\frac{\delta_{m}}{\Delta \omega}\right)^{2}\right]
$$

and the excitation probability amplitude $c_{e}^{(m)}$ consequently reads

$$
c_{e}^{(m)}=w_{m} \exp \left[i\left(\frac{\delta_{m}}{\Delta \omega}\right)^{2} a\right] .
$$

In Figure 3.2 we show the resulting excitation probability $\left|c_{e}^{(m)}\right|^{2}$ as a function of the chirp parameter $\phi^{\prime \prime}$. The excited state population displays oscillations for negative chirps, whereas for positive values of the chirp it is exponentially small. The oscillations are a result of the interference of two different excitation mechanisms: The direct path consists of an instantaneous absorption of two photons of frequency $\omega_{0}$. In contrast, the indirect path consists of two steps: first an excitation to the level $|m\rangle$ and second a transition to $|e\rangle$ which is delayed with respect to the first step.

In the next subsection we derive a phase space argument for the appearance of the quadratic phase factor in $c_{e}^{(m)}$.

\subsubsection{Interference of quantum paths}

Unfortunately, the analytical expression Eq. (3.37) for the transition probability amplitude does not reveal the origin of the quadratic phase factors. In the present section we identify the two crucial elements leading to the quadratic phase factor in $c_{e}^{(m)}$ :

(i) The interference of two kinds of excitation paths: the direct and the sequential path.

(ii) The sequential path is characterized by a phase which depends quadratically on the offset $\delta_{m}$.

\subsubsection{Origin of quadratic phases}

According to Eq. (3.25) the transition probability amplitude $c_{e}^{(m)}$ is determined by the integral $\mathcal{I}_{m}$. The latter is the product of two integrals which reflects the sequential character of the excitation process: in the first step, the atom is promoted to the intermediate state $|m\rangle$, while the second step provides the residual energy to reach the excited state. In order to bring out this feature most clearly 


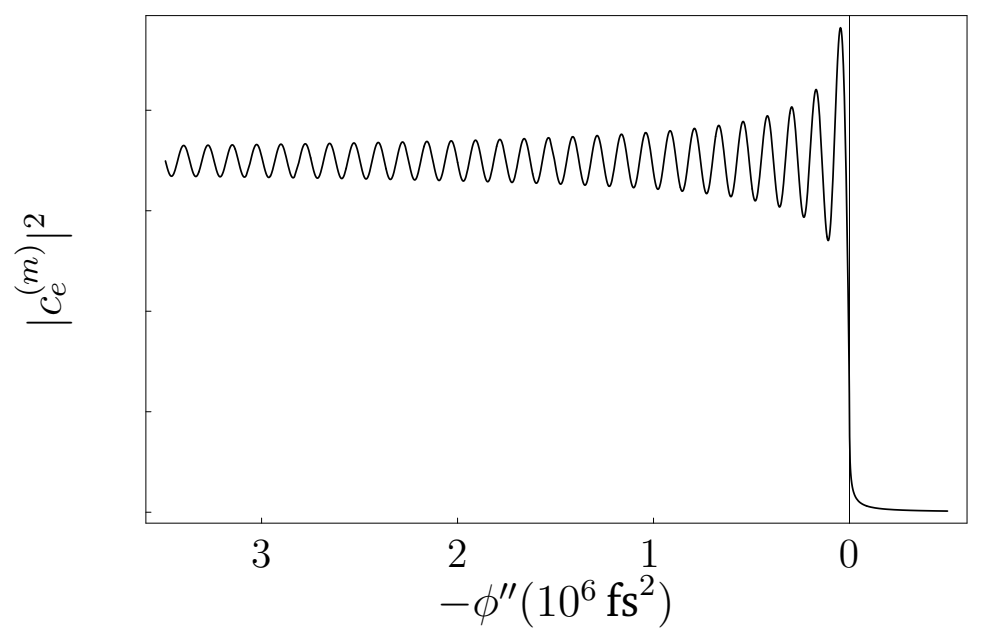

Figure 3.2: Chirping a two-photon excitation via a single intermediate state.

We show the resulting excitation probability $\left|c_{e}^{(m)}\right|^{2}$ as a function of the chirp parameter $\phi^{\prime \prime}$. The excited state population displays oscillations for negative chirps, whereas for positive values of the chirp it is exponentially small. The oscillations result from the interference of a direct and a sequential excitation path. The direct path is an instantaneous two-photon transition through the virtual state of frequency $\omega_{0}$. The indirect path consists of two steps: first an excitation to the level $|m\rangle$ and second a delayed transition to $|e\rangle$. Here we have borrowed the parameters $\delta=0.0225 \mathrm{fs}^{-1}, \Delta \omega=0.1525 \mathrm{fs}^{-1}, \Omega_{e m} \Omega_{m g}=1$ from a closely related experiment [Cha03] where a chirped laser pulse drives a two-photon transition through two intermediate states in the four-state ladder of Sodium.

Reprinted figure with permission from W. MERKEL, H.MACK, E. LUTZ, B. GiRARD, G. G. PAulus and W. P. Schleich, Physical Review A, accepted for publication (2007). Copyright 2007 by the American Physical Society. 
and to identify the times when the transitions occur we now substitute the expression Eq. (3.3) for the shape $f(t)$ of the pulse into the definition Eq. (3.26) of $\mathcal{I}_{m}$, which leads us to the integral

$$
\begin{aligned}
\mathcal{I}_{m}= & f_{0}^{2} \exp \left(i \frac{\delta_{m}^{2}}{4 \alpha_{i}}\right) \int_{-\infty}^{\infty} d t^{\prime} \exp \left[-\alpha_{r} t^{2}\right] \exp \left[-i \alpha_{i}\left(t^{\prime}+\frac{\delta_{m}}{2 \alpha_{i}}\right)^{2}\right] \\
& \times \exp \left(i \frac{\delta_{m}^{2}}{4 \alpha_{i}}\right) \int_{-\infty}^{t^{\prime}} d t^{\prime \prime} \exp \left[-\alpha_{r} t^{\prime \prime 2}\right] \exp \left[-i \alpha_{i}\left(t^{\prime \prime}-\frac{\delta_{m}}{2 \alpha_{i}}\right)^{2}\right]
\end{aligned}
$$

So far the calculation is exact. We now recognize that for large values of the chirp parameter $a$ the definitions Eq. (3.4) and Eq. (3.5) of $\alpha_{r}$ and $\alpha_{i}$ provide us with the inequality

$$
\left|\frac{\alpha_{r}}{\alpha_{i}}\right|=\frac{1}{|a|} \ll 1
$$

Consequently, the real-valued Gaussians in the integral Eq. (3.40) are slowly varying compared to the oscillatory terms resulting from the quadratic phases. The main contributions to the integral emerge from the oscillatory terms and in particular from the neighborhood of the times

$$
t_{s}^{\prime \prime} \equiv \frac{\delta_{m}}{2 \alpha_{i}}=\frac{\delta_{m}}{\Delta \omega^{2}} a\left(1+\frac{1}{a^{2}}\right) \cong \frac{\delta_{m}}{\Delta \omega^{2}} a
$$

and

$$
t_{s}^{\prime}=-\frac{\delta_{m}}{2 \alpha_{i}}=-t_{s}^{\prime \prime}
$$

when the phase factors are slowly varying.

Therefore, the transition from the level $|g\rangle$ to the level $|m\rangle$ appears at the time $t^{\prime \prime}=t_{s}^{\prime \prime}$ followed by the transition from $|m\rangle$ to $|e\rangle$ at the time $t^{\prime}=t_{s}^{\prime}$. In order to ensure this time ordering enforced by the limits $t^{\prime \prime}<t^{\prime}$ of the integral $\mathcal{I}_{m}$ defined in Eq. (3.40) we need to have

$$
t_{s}^{\prime \prime}<t_{s}^{\prime}=-t_{s}^{\prime \prime}
$$

This inequality is only satisfied provided $t_{s}^{\prime \prime}$ is negative. From Eq. (3.42) we recognize that it is the product $\delta_{m} a$ of offset and dimensionless chirp which determines the sign of $t_{s}^{\prime \prime}$. Consequently, we only find points of slowly varying phase for $\delta_{m} a<0$. In the case $0<\delta_{m} a$ no such points exist and the integral $\mathcal{I}_{m}$ is exponentially small.

Moreover, this analysis of the integral $\mathcal{I}_{m}$ brings out most clearly that each transition is associated with a phase which is quadratic in the offset $\delta_{m}$. Since the phases accumulated in both steps of the transition are identical, we find 
that the total acquired phase in the two-photon transition is twice of that of the individual one-photon transition.

We can now evaluate the integrals in an approximate way by replacing the variables $t^{\prime}$ and $t^{\prime \prime}$ in the real-valued Gaussians by $t_{s}^{\prime}$ and $t_{s}^{\prime \prime}$ and factoring them out of the integral which yields

$$
\mathcal{I}_{m} \cong \Theta\left(-\delta_{m} a\right) f_{0}^{2}\left(\int_{-\infty}^{\infty} d u e^{i \alpha_{i} u^{2}}\right)^{2} \exp \left[-\frac{\alpha_{r}}{\alpha_{i}} \frac{\delta_{m}^{2}}{2 \alpha_{i}}\right] \exp \left(i \frac{\delta_{m}^{2}}{2 \alpha_{i}}\right)
$$

where we have also extended the integration over $t^{\prime \prime}$ to $+\infty$. Here the Heaviside step function $\Theta($.$) accounts for the fact that both excitation steps in the two-$ photon absorption occur in the right temporal order.

With the help of the integral relation [Sch01]

$$
\int_{-\infty}^{\infty} d u e^{-i \gamma u^{2}}=\sqrt{\frac{\pi}{i \gamma}}
$$

we find

$$
\mathcal{I}_{m} \approx \Theta\left(-\delta_{m} a\right) f_{0}^{2} \frac{\pi}{i \alpha_{i}} \exp \left[-\frac{\alpha_{r}}{\alpha_{i}} \frac{\delta_{m}^{2}}{2 \alpha_{i}}\right] \exp \left[i \frac{\delta_{m}^{2}}{2 \alpha_{i}}\right] .
$$

When we now take the limit of $1 \ll|a|$ in the definitions of $f_{0}$ and $\alpha_{i}$, Eq. (3.7) and Eq. (3.5), which yields $f_{0}^{2} \cong i / a$ and $\alpha_{i}=\Delta \omega^{2} /(2 a)$ we obtain together with $\alpha_{r} / \alpha_{i}=1 / a$ the final approximate expression

$$
\mathcal{I}_{m} \approx \frac{2 \pi}{\Delta \omega^{2}} \Theta\left(-\delta_{m} a\right) \exp \left[-\left(\frac{\delta_{m}}{\Delta \omega}\right)^{2}\right] \exp \left[i\left(\frac{\delta_{m}}{\Delta \omega}\right)^{2} a\right] .
$$

When we substitute this asymptotic expression for the integral $\mathcal{I}_{m}$ into Eq. (3.25) of the probability amplitude $c_{e}^{(m)}$ we arrive at Eq. (3.37) where the weight factors are given by

$$
w_{m}=-d_{m} \Theta\left(-\delta_{m} a\right) \exp \left[-\left(\frac{\delta_{m}}{\Delta \omega}\right)^{2}\right]
$$

This expression results from the exact formula Eq. (3.38) with the help of the asymptotic expansion, Eq. (E.20), of the complementary error function derived in Appendix E.

\subsubsection{Interference in time-frequency phase space}

This analysis of the transition probability amplitude in second order perturbation theory translates into an elementary geometrical representation in a phase 


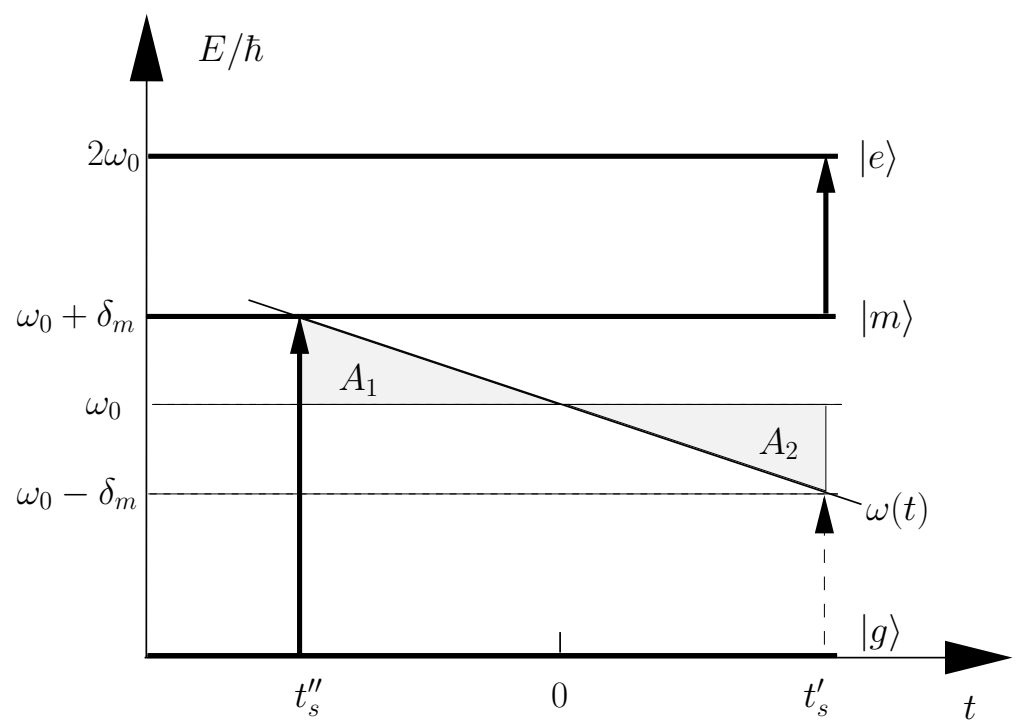

Figure 3.3: Origin of Gauss sum from phase space interpretation of two-photon excitation induced by a chirped pulse. In the phase space spanned by frequency $E / \hbar$ and time $t$ we indicate the ground state $|g\rangle$, the intermediate level $|m\rangle$ and the excited state $|e\rangle$ of frequencies $0, \omega_{0}+\delta_{m}$ and $2 \omega_{0}$, respectively, by thick horizontal lines. We also denote the one-photon resonance of energy $\omega_{0}$ and the virtual level of frequency $\omega_{0}-\delta_{m}$ by dashed horizontal lines. The instantaneous frequency $\omega(t)=\omega_{0}+2 \alpha_{i} t$ is depicted for a negative value of the chirp $\phi^{\prime \prime}$ by the tilted thin line. A stationary phase analysis of the double integral Eq. (3.26) determining the two-photon transition probability amplitude brings out most clearly that the sequential excitation appears at the times $-t_{s}$ and $+t_{s} \equiv$ $\left|\delta_{m} / \alpha_{i}\right|$. Indeed, at $-t_{s}$ the instantaneous frequency is resonant with the transition from $|g\rangle \rightarrow|m\rangle$ whereas at $+t_{s}$ it is resonant with the transition from $|m\rangle$ to $|e\rangle$ as indicated by the dashed and the thick arrow. In this phase space representation the times when the transitions occur are determined by the crossings of the tilted thin line with the thick and the lower dashed horizontal lines corresponding to the frequencies $\omega_{0}+\delta_{m}$ and $\omega_{0}-\delta_{m}$. The enclosed shaded area $A_{1}+A_{2}$ corresponds to the phase acquired during the transition. Each triangle has the area $\delta_{m} t_{s}^{\prime \prime} / 2=\delta_{m}^{2} /\left(2 \alpha_{i}\right)$. As a consequence, the phase associated with this transition is quadratic in the offset $\delta_{m}$.

Reprinted figure with permission from W. MERKEL, H.MACK, E. LUTZ, B. GiRARD, G. G. PAulus and W. P. SChleich, Physical Review A, accepted for publication (2007). Copyright 2007 by the American Physical Society. 


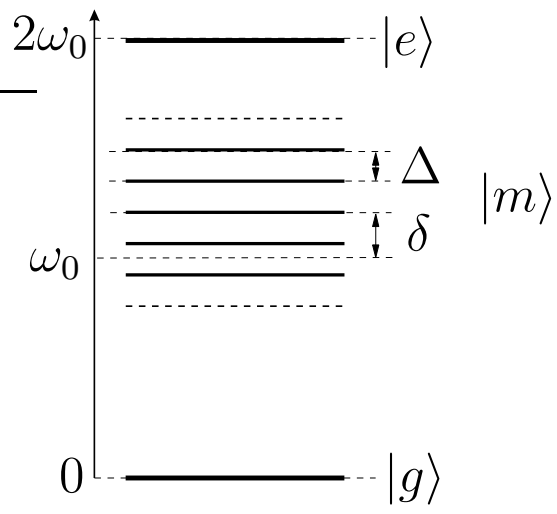

Figure 3.4: Extended ladder system: The ground state $|g\rangle$ is connected by a two-photon transition to the excited state $|e\rangle$. As an extension of the three-state ladder system of Figure 3.1 we include a harmonic manifold of $D=M^{\prime}+M+1$ intermediate states $|m\rangle$ (with $-M^{\prime} \leq m \leq M$ ) which are shifted by the offset $\delta_{m}=\delta+m \Delta$ with respect to the central frequency $\omega_{0}$. The offset of the central state is $\delta$ whereas the states in the harmonic manifold are separated by $\Delta$.

space spanned by frequency $E / \hbar$ and time $t$. In Figure 3.3 we consider the sequential excitation path via the state $|m\rangle$ in this space. We indicate the frequencies $0, \omega_{0}+\delta_{m}$ and $2 \omega_{0}$ of the ground state, the intermediate state and the excited state by thick horizontal lines. We also denote the one-photon resonance of energy $\omega_{0}$ and the virtual level of frequency $\omega_{0}-\delta_{m}$ by dashed horizontal lines. The instantaneous frequency $\omega(t)=\omega_{0}+2 \alpha_{i} t$ is depicted for one negative value of the chirp by the tilted thin line.

The transitions from $|g\rangle$ to $|m\rangle$ and from $|m\rangle$ to $|e\rangle$ occur at times $-t_{s}$ and $+t_{s}$, respectively. These times are determined geometrically by the crossings of the representations of the instantaneous frequency $\omega(t)$ and the two frequencies $\omega_{0}+\delta_{m}$ and $\omega_{0}-\delta_{m}$, that is, by the crossings of the tilted thin line with the thick and the lower dashed horizontal lines. Each crossing contributes to the total transition probability amplitude with a phase determined by the enclosed triangular shaded area $A_{1}+A_{2}$ corresponding to the phase acquired during the transition. Each triangle has the area $\delta_{m} t_{s}^{\prime \prime} / 2=\delta_{m}^{2} /\left(2 \alpha_{i}\right)$. As a consequence, the phase associated with this transition is quadratic in the offset $\delta_{m}$.

\subsection{Chirped pulse excitation in a multistate ladder}

In the previous section we have investigated the two-photon excitation in a three-state ladder induced by a weak chirped laser pulse. Moreover, we have shown that for a certain choice of parameters the sequential excitation path is accompanied by an accrual of a quadratic phase factor.

Now we extend the simple three-state ladder of the previous section by 
taking into account a harmonic manifold of equidistant intermediate states $|m\rangle$ with quantum number $-M^{\prime} \leq m \leq M$, as depicted in Figure 3.4. The offset of these intermediate states is given by

$$
\delta_{m}=\delta+m \Delta .
$$

The harmonic manifold is characterized by the offset $\delta$ of a specific central state $|0\rangle$ and an equidistant spacing $\hbar \Delta$ between neighboring states.

From our previous considerations we find that only intermediate states with $0<\delta_{m}$ take part in the excitation process when driven by a negatively chirped laser pulse $(a<0)$. Consequently, all participating intermediate states are characterized by quantum numbers

$$
m>-\frac{\delta}{\Delta}
$$

Each state in the harmonic manifold is coupled to both the ground and the excited state. Transitions between states of the harmonic manifold are forbidden.

As a consequence, we extend the previous RWA interaction Hamiltonian, Eq. (3.17), to multiple intermediate states according to

$$
\mathcal{V}(t) \equiv \sum_{m=-M^{\prime}}^{M} \mathcal{V}^{(m)}(t)=\sum_{m=-M^{\prime}}^{M} \mathcal{V}_{g m}(t)+\mathcal{V}_{e m}(t)
$$

Thus we account for all possible transitions in this extended ladder.

\subsubsection{Exact expression for the total excitation probability ampli- tude}

In order to obtain the total excitation probability amplitude

$$
c_{e} \equiv \sum_{m=-M^{\prime}}^{M} c_{e}^{(m)}
$$

we have to sum over all possible contributions $c_{e}^{(m)}$ associated with each single excitation path connecting $|g\rangle$ and $|e\rangle$ by passing through the $m$-th intermediate state. Here we point out that the evaluation of each contribution proceeds along the same lines as shown in the previous section.

In the last section we showed that such quantum paths are accompanied by the accrual of a quadratic phase factor. Here the accumulated phase is quadratic in the offset $\delta_{m}$. 
In the present case of the multi-state ladder with a harmonic manifold of intermediate states we have the interference of all excitation paths, that is, the weighted sum of quadratic phase factors giving rise to a Gauss sum.

With the help of the offset $\delta_{m}$, Eq. (3.50), we rewrite the relevant quadratic phase in Eq. (3.37) as

$$
\left(\frac{\delta_{m}}{\Delta \omega}\right)^{2} a=\frac{\pi}{2} N \xi+2 \pi\left(m+\frac{m^{2}}{N}\right) \xi
$$

where we define the number

$$
N=\frac{2 \delta}{\Delta}
$$

and the argument (dimensionless chirp)

$$
\xi=\frac{\delta \Delta}{\pi} \frac{a}{\Delta \omega^{2}}=\frac{\delta \Delta}{\pi} \phi^{\prime \prime}
$$

which depend on the parameters $\delta$ and $\Delta$ of the harmonic manifold. Moreover, we express the offset

$$
\delta_{m}=\Delta\left(m+\frac{\delta}{\Delta}\right)=\Delta\left(m+\frac{N}{2}\right)
$$

in terms of $N$. Hence, the final expression for the excited state probability amplitude takes the form

$$
c_{e}=\exp \left(i \frac{\pi}{2} N \xi\right) \sum_{m=-M^{\prime}}^{M} w_{m} \exp \left[2 \pi i\left(m+\frac{m^{2}}{N}\right) \xi\right]
$$

with the weight factors

$$
w_{m} \equiv-\frac{d_{m}}{2} \operatorname{erfc}\left(\zeta_{m}\right) \exp \left[-\left(\frac{m+N / 2}{\Delta m}\right)^{2}\right] .
$$

Here we have introduced the abbreviation

$$
\zeta_{m} \equiv \frac{m+N / 2}{\Delta m} i \sqrt{1-i a}
$$

and the width

$$
\Delta m \equiv \frac{\Delta \omega}{\Delta} .
$$

Thus in the multi-state ladder system the total transition probability amplitude $c_{e}$ consists of a weighted Gauss sum over phase factors with phases that depend linearly and quadratically on the summation index $m$.

In Figure 3.5 we depict the excited state population $\left|c_{e}\right|^{2}$ which arises from the interference of competing quantum paths via the intermediate states $|m\rangle$. Due to the quadratic phase characteristic to each sequential quantum path we find strong modulations as a function of the chirp. In the domain of positive values of $\phi^{\prime \prime}$ sequential excitation is not possible such that the excited state probability decays like an exponential. 


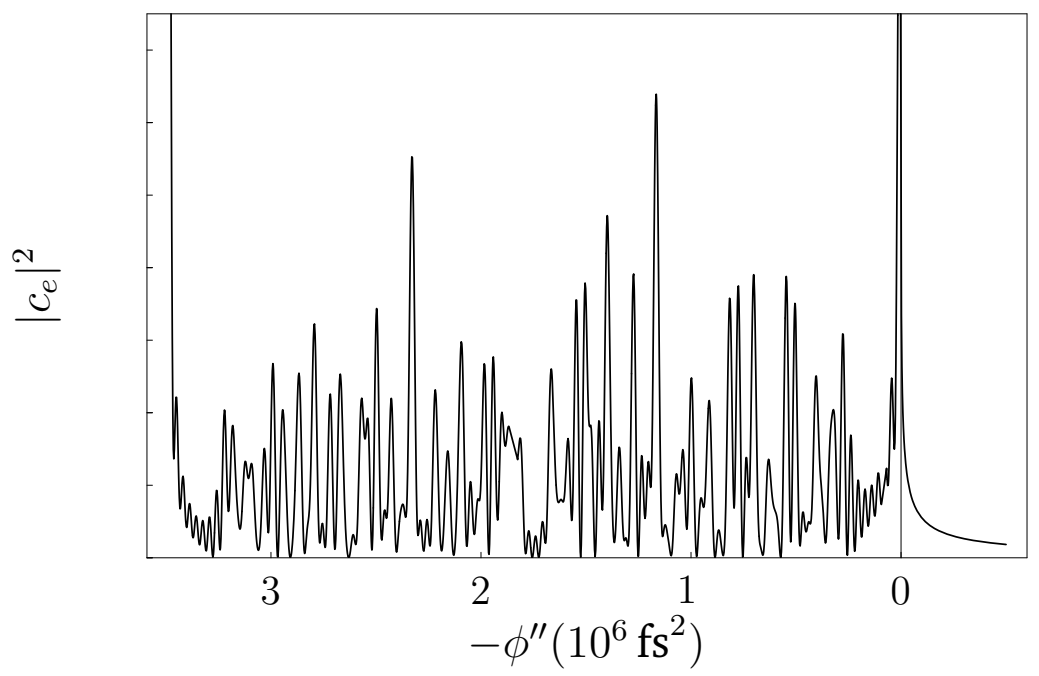

Figure 3.5: Chirping a two-photon excitation through an equidistant manifold of intermediate states.

The population $\left|c_{e}\right|^{2}$ of the excited state arises due to interference of competing quantum paths via 15 intermediate states $|m\rangle$ and displays strong modulations as a function of the chirp. Here we have made the idealized assumption that all two-photon transitions $|g\rangle \rightarrow|m\rangle \rightarrow|e\rangle$ appear with equal weight, that is $\Omega_{e m} \Omega_{m g}=1$. The other parameters are chosen according to $M^{\prime}=M=7, \delta=0.0225 \mathrm{fs}^{-1}, \Delta=0.003 \mathrm{fs}^{-1}, \Delta \omega=0.1525 \mathrm{fs}^{-1}$.

Reprinted figure with permission from W. MERKEL, H.MACK, E. LUTZ, B. GIRARD, G. G. PAulus and W. P. Schleich, Physical Review A, accepted for publication (2007). Copyright 2007 by the American Physical Society. 


\subsubsection{Asymptotic expansion of weight factors}

The weight factors $w_{m}$ given by Eq. (3.59) follow from the complementary error function with a complex argument $\zeta_{m}$. Therefore it is difficult to gain insight into their dependence on the summation index $m$. For this reason we now derive an approximate analytical expression for $w_{m}$ in the limit of large positive or negative values of the dimensionless chirp $a$. form

In this case the argument $\zeta_{m}$ of the complementary error function takes the

$$
\zeta_{m} \equiv z_{r}+i z_{i} \approx \frac{m+N / 2}{\Delta m} \sqrt{a} e^{i \beta}
$$

where

$$
\beta \equiv \frac{\pi}{2}\left(1-\frac{\operatorname{sign}(a)}{2}\right) .
$$

In Appendix E we derive the asymptotic expansion

$$
\operatorname{erfc}\left(\zeta_{m}\right) \stackrel{\sim}{\cong} \Theta\left(-\delta_{m} a\right)
$$

in the limit of large values of $|a|$ where $\Theta$ denotes the Heaviside step function. As a consequence we arrive at the asymptotic formula

$$
w_{m} \approx-d_{m} \Theta\left[-a\left(m+\frac{N}{2}\right)\right] \exp \left[-\left(\frac{m+N / 2}{\Delta m}\right)^{2}\right] .
$$

Hence, the weight factors are one wing of a Gaussian centered around $m \approx$ $-N / 2$. The sign of the chirp determines which wing. Indeed, for negative chirps, that is $a<0$ we only find contributions for $m>-N / 2$. In contrast for positive chirps, that is $0<a$, only levels with $m<-N / 2$ contribute. Moreover, by controlling the product of Rabi frequencies entering $d_{m}$, Eq. (3.36), and the width $\Delta m$ we can ensure that all quantum paths contribute to the sum with approximately the same weight.

\subsection{Candidate systems for an experimental realiza- tion}

In the previous sections we have shown that a specific ladder driven by a chirped pulse leads to a Gauss sum in the excitation probability amplitude. Goal of this section is to discuss standard quantum optical systems in the context of realizing such an equidistant ladder.

Before we address this task it is helpful to recall the origin of the Gauss sum - the interference of paths with quadratic phases. Figure 3.3 brings out most clearly that these phases arise already from the first step of the excitation, that 


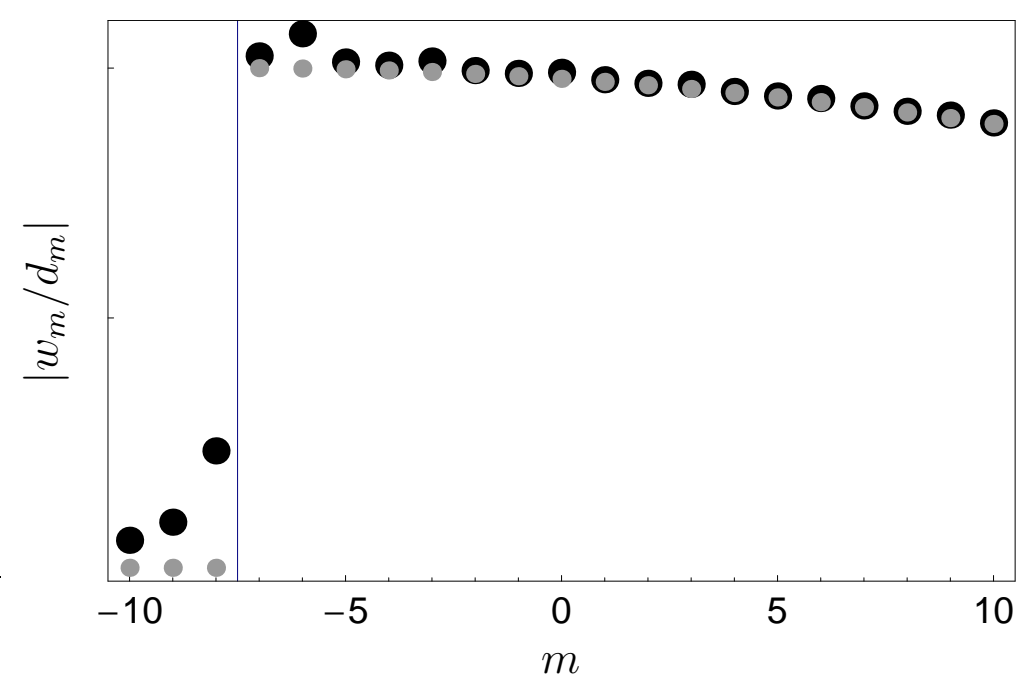

Figure 3.6: Comparison between the exact (black dots) and the approximate (gray dots) expression for the weight factors $\left|w_{m} / d_{m}\right|$ given by Eqs. (3.59) and (3.65), respectively. Here we consider the case $N=15$ with $\Delta m=\Delta \omega / \Delta=50.8$ for negative dimensionless chirps $a=-10824<0$.

Reprinted figure with permission from W. MERKEL, H.MACK, E. LUTZ, B. GIRARD, G. G. Paulus and W. P. Schleich, Physical Review A, accepted for publication (2007). Copyright 2007 by the American Physical Society.

is, from transition into the equidistant manifold. Therefore, a one-photon transition from a ground state to an equidistant manifold of excited states driven by a chirped pulse also leads to a Gauss sum. In this simplified system the fluorescence signal is acquired from the states in the harmonic manifold decaying to the ground state. Interference from the individual levels occurs during spontaneous emission of the states.

At least five different quantum systems offer themselves as candidates for such one-photon excitations:

- Rydberg atoms in electric or magnetic fields,

- vibrational energy levels in a molecule,

- atoms in traps,

- quantum dots, and

- laser-driven one-photon transitions.

However, we subject these systems to additional requirements:

(i) The Rabi-frequencies for the interfering excitation paths should be slowly varying over a sufficiently wide range of intermediate states $|m\rangle$. Of 
Gauss sums arising from a chirped two-photon transition

course, in the most ideal ideal situation the Rabi-frequencies are of the same order of magnitude and all excitation paths enter the sum with the same weight. For most physical systems this condition is hard to meet. Therefore, in each case the effects of the particular weight factor distribution on the factorization property have to be analyzed.

(ii) Since anharmonicities in the target manifold change the character of the resulting probability amplitude, only equidistant states result in a perfect Gauss sum.

(iii) In the our derivation we have assumed that the time scale of the chirped pulse excitation is shorter compared to the intrinsic time scale of spontaneous decay processes in each system.

We now briefly discuss these quantum systems in the context of of these requirements. A detailed discussion is beyond the scope of this work and would require a careful analysis of the subtleties of each system.

Rydberg atoms [Gal88] are highly sensitive to electric or magnetic fields. In an electric field the energy levels of a Rydberg atom split into a manifold of states [Zim79]. The so-called Stark maps, which show the dependence of the splitted spectral lines as a function of the electric field, have been measured for many alkali atoms. Whereas for a modest field strength the separation between neighboring splitted energy levels is constant, for larger values of the field also higher order corrections to the energy have to be taken into account. A closer analysis of the Stark map reveals that the dipole moments vary for each member of the manifold and the individual paths do not contribute equally. Due to the Zeeman effect a homogeneous magnetic field also creates a manifold of equidistant states $|m\rangle$. However, in this case the problem is the selection rules which eliminate most of the transitions. A change of the orientation of the magnetic field might offer a possibility to overcome this problem.

Diatomic molecules might also serve as a candidate system for identifying a manifold of (almost) equidistant states [Wal02]. The diagonalization of the ro-vibrational Hamiltonian yields that the inter-nuclear potential in a diatomic molecule can be indeed approximated as a harmonic oscillator with equidistant spectrum. However, anharmonicities would certainly have negative effects on the regularity of the Gauss sum.

A more promising realization of an equidistant spectrum relies on cold trapped atoms or ions [Phi98, Wie99, Lei03]. The central idea of this approach is that the trapping in magnetic microtraps relies on the magnetic dipole of the atom, which depends on its internal state. Therefore, the center-of-mass motion of an atom depends on its internal state. Our suggestion for the realization of an equidistant spectrum is reminiscent of the quantum tweezer [Die02, Moh05]. Whereas the atom initially in the ground state is weakly bound to a shallow trap, the atom in its excited state experiences a steep harmonic potential. The 
vibrational states in the excited electronic state constitute the desired harmonic manifold. An advantage of this realization consists in the high level of control which would allow to adapt the factorization scheme to a wider range of numbers $N$.

Quantum dots [Ash96] may be the perfect choice in the end. Indeed, they allow for designing the discrete energy spectrum [Oos98] of a trapped electron and can thus be viewed as an artificial atom. Quantum dots have already been discussed as a candidate system for implementing quantum logic [Los98].

Yet another method to obtain an equidistant ladder system is to apply two electromagnetic fields to a two-level atom which is characterized by a permanent dipole moment in the excited state. A strong cw-modulation field generates an equidistant Floquet ladder in the excited state. The second field, which is again a weak chirped pulse, induces an excitation probability amplitude of the form of a Gauss sum.

\subsection{Discussion}

In this chapter we have shown that chirping a two-photon transition in a threestate ladder through a single intermediate state induces in the weak field limit an excitation probability amplitude which is proportional to a phase factor that is quadratic in the offset of the intermediate state.

For a generalized ladder system containing a manifold of equidistant intermediate states the total excitation probability amplitude is proportional to a Gauss sum.

Moreover, we have briefly discussed several quantum systems in the context of realizing a Gauss sum in the excitation probability amplitude by means of a chirped laser pulse.

In the next chapter we specialize on the most promising candidate, which is based on laser-driven one-photon transitions in two-level systems. We provide two approaches to engineer Gauss sums which experimental realization is more straightforward. 


\section{CHAPTER 4}

\section{Gauss sums arising from laser-driven one-photon transitions}

In chapter 3 we have shown that the excitation probability amplitude of a twophoton transition through a multi-ladder system driven by a linearly chirped pulse takes on the form of a Gauss sum.

Whereas in in the last chapter we have concentrated on the general principles and have only briefly discussed the physical implementations of such ladder systems we devote this chapter to a detailed discussion of realizing Gauss sums on the basis of a laser-driven two-level system. In particular we suggest two approaches on the basis of a one-photon transition in laser-driven two-level systems. Both approaches rely on quantum interference of multiple quantum paths. However, they differ in the origin of the quadratic phase factor.

We consider a two-level system with permanent dipole moment in the excited state. In the first approach a continuous wave (cw) microwave field drives the excited state and induces an equidistant set of sidebands. If this one-photon transition is excited from the ground state by a chirped laser pulse, the resulting excited state probability amplitude is the sum over all possible excitation channels involving one optical photon and multiple quanta of the microwave field. As in the previous chapter the Gauss sum arises due to the quadratic phase (chirp) of the laser pulse.

The second approach relies on multi-pulse excitation of a two-level system, which is characterized by a linearly chirped resonance condition. The accumulated excited state probability amplitude is the superposition of contributions arising from each individual pulse. The quadratic phase dependence appears solely due to the linear Stark shift of the excited state.

This chapter is organized as follows: In section 4.1 we investigate the model 
of one-photon excitation in a two-level system. We apply this model in section 4.2 to the first realization which is based on a two-level system with a permanent dipole moment driven by a microwave field and a chirped laser pulse. Section 4.3 is devoted to the analysis of a linearly modulated transition which is driven by a sequence of short pulses.

\subsection{Model}

We consider a two-level system with ground state $|g\rangle$ and excited state $|e\rangle$ separated by the energy difference $\hbar \omega_{0}$. The permanent dipole moment $\wp_{e e}$ of the excited state interacts with the modulation field $E_{\mathrm{m}}(t)$, which gives rise to a time-dependent shift of the energy level. We consider two cases for $E_{\mathrm{m}}(t)$ :

(i) a sinusoidal microwave field manifesting itself in a periodic modulation of the excited state and

(ii) a quadratic chirp reflecting itself in a linear shift of the excited state energy.

In addition, we have a second time-dependent weak electric field $E_{\mathrm{d}}(t)$ which drives the transition between the ground and excited state. Depending on the two cases $E_{\mathrm{d}}(t)$ is either a

(i) chirped laser pulse $E_{\mathrm{d}}=E_{\mathrm{c}}$ or

(ii) a sequence of short and distinct laser pulses.

We account for this combination of electric fields by the interaction Hamiltonian

$$
V=-\wp_{e e}|e\rangle\langle e| E_{\mathrm{m}}(t)-\left(\wp_{g e}|e\rangle\langle g|+\text { c.c. }\right) E_{\mathrm{d}}(t),
$$

where $\wp_{g e}$ is the dipole moment of the dipole induced by the electric field

$$
E_{\mathrm{d}}(t)=\mathcal{E}_{0}\left[e^{-i \omega_{L} t} f(t)+\text { c.c. }\right]
$$

with amplitude $\mathcal{E}_{0}$, optical carrier frequency $\omega_{L}$ and pulse shape $f(t)$. Here we assumed that the frequencies of the two fields are clearly separated. In this case the modulation field $E_{\mathrm{m}}(t)$ acts only on the excited state and the field $E_{\mathrm{d}}(t)$ only on the transition.

In the interaction picture the time evolution of the probability amplitudes $c_{e}(t)$ and $c_{g}(t)$ for being in the excited or ground state read

$$
\begin{aligned}
& i \frac{d}{d t} c_{e}(t)=-\Omega_{e e}(t) c_{e}(t)-\Omega_{g e}(t) e^{i \omega_{0} t} c_{g}(t) \\
& i \frac{d}{d t} c_{g}(t)=-\Omega_{e g}(t) e^{-i \omega_{0} t} c_{e}(t)
\end{aligned}
$$


where we define the time-dependent Rabi-frequencies

$$
\Omega_{e e}(t) \equiv \frac{\wp_{e e} E_{\mathrm{m}}(t)}{\hbar} \quad \text { and } \quad \Omega_{e g}(t) \equiv \frac{\wp_{e g} E_{\mathrm{d}}(t)}{\hbar}
$$

associated with both electric fields.

We neglect spontaneous emission since we assume the interaction time with the driving field to be much shorter than the characteristic decay time of the atomic level. This fact allows us to describe the system by the Schrödinger equation rather than a density matrix.

In order to solve the Schrödinger equation we recall that the strong field $E_{\mathrm{m}}(t)$ causing the modulation of the excited state appears in the diagonal element of Eq. (4.1). Only the weak laser field with Rabi frequency $\Omega_{g e}(t)\left[\Omega_{e g}(t)\right]$ couples the off-diagonal elements leading to transitions. For this reason it suffices to apply perturbation theory of first order.

In the weak field limit the state of the two-level system initially occupies the ground level, that is

$$
c_{g}\left(t_{0}\right)=1 \text { and } c_{e}\left(t_{0}\right)=0 .
$$

We assume that the excited state probability amplitude does not change significantly under the action of a weak chirped pulse which yields $c_{g}(t) \approx 1$. Hence we are left with the single inhomogeneous differential equation

$$
i \frac{d}{d t} c_{e}(t)=-\Omega_{e e}(t) c_{e}(t)-\Omega_{g e}(t) e^{i \omega_{0} t}
$$

for the excited state probability amplitude.

The interaction with the chirped laser pulse acts as an inhomogeneity and it is easy to verify that the solution reads

$$
c_{e}(t)=i e^{i \beta(t)} \int_{t_{0}}^{t} d t^{\prime} \exp \left[-i \beta\left(t^{\prime}\right)\right] \exp \left[i \omega_{0} t^{\prime}\right] \Omega_{g e}\left(t^{\prime}\right)
$$

where we have introduced the phase

$$
\beta(t) \equiv \int_{t_{0}}^{t} d t^{\prime} \Omega_{e e}\left(t^{\prime}\right)
$$

When we substitute the electric field $E_{\mathrm{d}}(t)$, Eq. (4.2), into the solution Eq. (4.7) for the probability amplitude $c_{e}$ we find in rotating wave approximation

$$
c_{e}(t)=i \Omega_{g e} e^{i \beta(t)} \int_{t_{0}}^{t} d t^{\prime} \exp \left[-i \beta\left(t^{\prime}\right)\right] \exp \left[i \delta t^{\prime}\right] f\left(t^{\prime}\right) .
$$




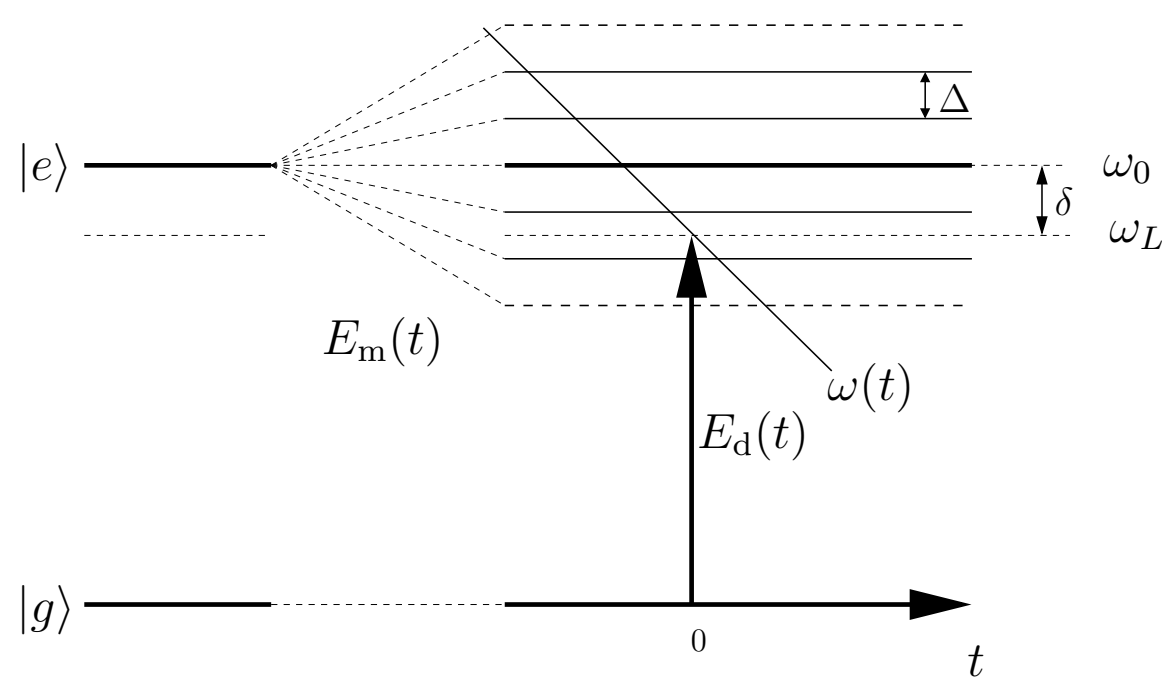

Figure 4.1: Engineering the Floquet ladder. We consider a two-level system with the ground state $|g\rangle$ and the excited state $|e\rangle$ separated by the energy $\hbar \omega_{0}$. The excited state is modulated by a strong sinusoidal field $E_{m}(t)$, Eq. (4.11), giving rise to equidistant sidebands separated by $\hbar \Delta$. Here each sideband appears weighted by the Bessel function $J_{n}(\kappa)$. The one-photon transition is driven by a chirped laser pulse $E_{d}(t), E q$. (4.2), which is characterized by a linear variation of the instantaneous frequency $\omega(t)$.

Reprinted figure from W. Merkel, I. Sh. Averbukh, B. Girard, G. G. Paulus and W. P. SCHLEICH, "Factorization of numbers with physical systems", Fortschritte der Physik 54, Nos. 8-10, p. 856-865 (2006). Copyright Wiley-VCH Verlag GmbH \& Co. KGaA. Reproduced with permission.

Here we define the time-independent Rabi-frequency

$$
\Omega_{g e} \equiv \frac{\wp_{g e} \mathcal{E}_{0}}{\hbar}
$$

associated with the electric field of the transfer pulse and also the detuning $\delta \equiv \omega_{0}-\omega_{L}$ between the atomic and the carrier frequency.

In the following sections we apply the results of the present section in order to calculate the excitation probability amplitude $c_{e}$ for both choices (i) and (ii) of the electric fields $E_{\mathrm{m}}$ and $E_{\mathrm{d}}$.

\subsection{Floquet ladder}

The key idea of this approach bases on the periodic modulation of the excited state by a strong continuous wave. Here the excited state is modulated by equidistant satellites. 


\subsubsection{Excitation probability in the weak field limit}

In the case $(i)$ of the cw modulation field

$$
E_{\mathrm{m}}(t)=\mathcal{F}_{0} \cos (\Delta t+\varphi),
$$

with period $2 \pi / \Delta$, amplitude $\mathcal{F}_{0}$ and phase $\varphi$, the phase $\beta(t)$ defined in Eq. (4.8) reads

$$
\beta(t) \equiv \kappa \sin (\Delta t+\varphi) .
$$

For simplicity we have chosen the lower integration limit according to $t_{0}=$ $-\varphi / \Delta$. We have also introduced the dimensionless quantity

$$
\kappa \equiv \frac{\Omega_{e e}}{\Delta}
$$

where

$$
\Omega_{e e} \equiv \frac{\wp_{e e} \mathcal{F}_{0}}{\hbar}
$$

stands for the time-independent Rabi-frequency. When we apply the Bessel function decomposition[Abr72]

$$
\exp (i \kappa \sin \theta)=\sum_{n=-\infty}^{\infty} J_{n}(\kappa) e^{i n \theta}
$$

to the phase factor in the integrand of Eq. (4.7) we obtain the expression

$$
c_{e}(t)=i \Omega_{g e} e^{i \beta(t)} \sum_{n} J_{n}(\kappa) e^{-i n \varphi} \tilde{f}_{n}(t)
$$

for the excitation probability amplitude. Here we have interchanged the order of integration and summation and have also introduced the integral

$$
\tilde{f}_{n}(t) \equiv \int_{t_{0}}^{t} d t^{\prime} \exp \left[i \delta_{n} t^{\prime}\right] f\left(t^{\prime}\right)
$$

with the offset

$$
\delta_{n} \equiv \delta-n \Delta
$$

characterizing the $n$-th satellite of the excited state.

This result can be interpreted in the way that the cw driving field causes a modulation of the energy of the excited state by equidistant sidebands where the associated Bessel function $J_{n}(\kappa)$ determines the weight of the $n$-th sideband. In the language of Floquet theory [Shi65, Sho90, Gri97] we have replaced the time-dependent Hamiltonian by an infinite dimensional Floquet matrix. 


\subsubsection{Excitation probability for a chirped pulse}

So far our calculation is valid for arbitrary pulse shapes. We now perform the integration in Eq. (4.17) for the case of a chirped pulse $E_{\mathrm{d}}(t)=E_{\mathrm{c}}(t)$ with a complex-valued envelope $f(t)$ given by Eq. (3.3).

We are interested in times after the pulse has interacted, that is for $t \gg$ $\sqrt{1+a^{2}} / \Delta \omega$. In addition, it is also feasible to extend the lower integration limit $t_{0}$ to $-\infty$. Thus we arrive at the integral

$$
\tilde{f}_{n}(\infty) \equiv f_{0} \int_{-\infty}^{\infty} d t^{\prime} \exp \left[-\frac{1}{2}\left(\Delta \omega f_{0} t^{\prime}\right)^{2}-i \delta_{n} t^{\prime}\right]
$$

which yields

$$
\tilde{f}_{n}(\infty)=\frac{\sqrt{2 \pi}}{\Delta \omega} \exp \left[-\frac{1}{2 f_{0}^{2}}\left(\frac{\delta_{n}}{\Delta \omega}\right)^{2}\right]
$$

When we recall the definition Eq. (3.7) of $f_{0}$ we decompose the Gaussian in $\tilde{f}_{n}$ into a real-valued one and into a quadratic phase factor, that is

$$
\tilde{f}_{n}(\infty)=\frac{\sqrt{2 \pi}}{\Delta \omega} \exp \left[-\frac{1}{2}\left(\frac{\delta_{n}}{\Delta \omega}\right)^{2}\right] \exp \left[i\left(\frac{\delta_{n}}{\Delta \omega}\right)^{2} \frac{a}{2}\right]
$$

Hence, in the long-time limit the excitation probability amplitude takes on the form

$$
c_{e}(t)=i 2 \pi \frac{\Omega_{g e}}{\Delta} e^{i \beta(t)} \exp \left(i \frac{\pi}{2} N^{\prime} \xi^{\prime}\right) \sum_{n} w_{n}(\varphi) \exp \left[-2 \pi i\left(n-\frac{n^{2}}{N^{\prime}}\right) \xi^{\prime}\right]
$$

where we have introduced the dimensionless quantity

$$
N^{\prime} \equiv \frac{2 \delta}{\Delta}
$$

the rescaled chirp

$$
\xi^{\prime} \equiv \frac{\delta \Delta}{2 \pi} \phi^{\prime \prime}
$$

and the weight factors

$$
w_{n}(\varphi) \equiv e^{-i n \varphi} J_{n}(\kappa) g_{n}\left(N^{\prime}, \Delta n\right) .
$$

The latter consist of a product of the Gaussian

$$
g_{n}\left(N^{\prime}, \Delta n\right)=\frac{1}{\sqrt{2 \pi} \Delta n} \exp \left[-\frac{1}{2}\left(\frac{n-N^{\prime} / 2}{\Delta n}\right)^{2}\right]
$$


of width

$$
\Delta n \equiv \frac{\Delta \omega}{\Delta}
$$

and the Bessel function $J_{n}(\kappa)$ together with the phase factors $\exp (-i n \varphi)$. The width $\Delta n$ of the Gaussian can be controlled by the choice of the parameters of the laser fields $E_{\mathrm{m}}(t)$, Eq. (4.11), and $E_{\mathrm{c}}(t)$, Eq. (3.3), respectively.

In the present physical system of a chirped Floquet ladder the resulting excitation probability amplitude, Eq. (4.22), again contains a weighted sum over quadratic phase factors. In the following we address the influence of the weight factors $w_{n}(\varphi)$ in more detail.

\subsubsection{Choice of modulation index}

In the preceding section we have derived an expression for the excitation probability induced by a chirped pulse, which contains a weighted sum over quadratic phase factors. However, it is not clear yet that this sum really has the ability to factorize numbers. In order to show this ability we still have to choose the appropriate parameters for the phase $\varphi$ and the modulation index $\kappa$.

In order to reduce the influence of the Bessel functions in the weight factors, Eq. (4.25), the modulation index

$$
\kappa=\frac{\Omega_{e e}}{\Delta}=\frac{\wp_{e e} \mathcal{F}_{0}}{\hbar \Delta}
$$

provided by the microwave field, Eq. (4.11), has to be adjusted such that the weight factors $w_{n}(\varphi)$ are slowly varying as a function of $n$. For this purpose we recall the asymptotic expansion [Abr72]

$$
J_{n}(z) \cong \sqrt{\frac{2}{\pi z}} \cos \left(z-n \frac{\pi}{2}-\frac{\pi}{4}\right)
$$

for the Bessel function in the limit of large arguments $z \gg n$ which suggests the choice of the argument

$$
\kappa=2 \pi s+\frac{\pi}{4}
$$

where $s$ is a large integer. From the experimental point of view such a choice of $\kappa$ can be realized by adjusting the amplitude $\mathcal{F}_{0}$ of the cw driving field.

With this special choice of $\kappa$ we find

$$
J_{n}(\kappa) \cong \sqrt{\frac{2}{\pi \kappa}} \cos \left(m \frac{\pi}{2}\right)=\sqrt{\frac{2}{\pi \kappa}}\left\{\begin{array}{cl}
(-1)^{m} & \text { for } n=2 m \\
0 & \text { for } n=2 m+1,
\end{array}\right.
$$

and thus all weight factors with odd index $n$ vanish and we are left with the non-zero weight factors

$$
w_{2 m}(\varphi) \approx \sqrt{\frac{2}{\pi \kappa}} e^{i m(\pi-2 \varphi)} g_{m}\left(\frac{N^{\prime}}{2}, \frac{\Delta n}{2}\right)
$$



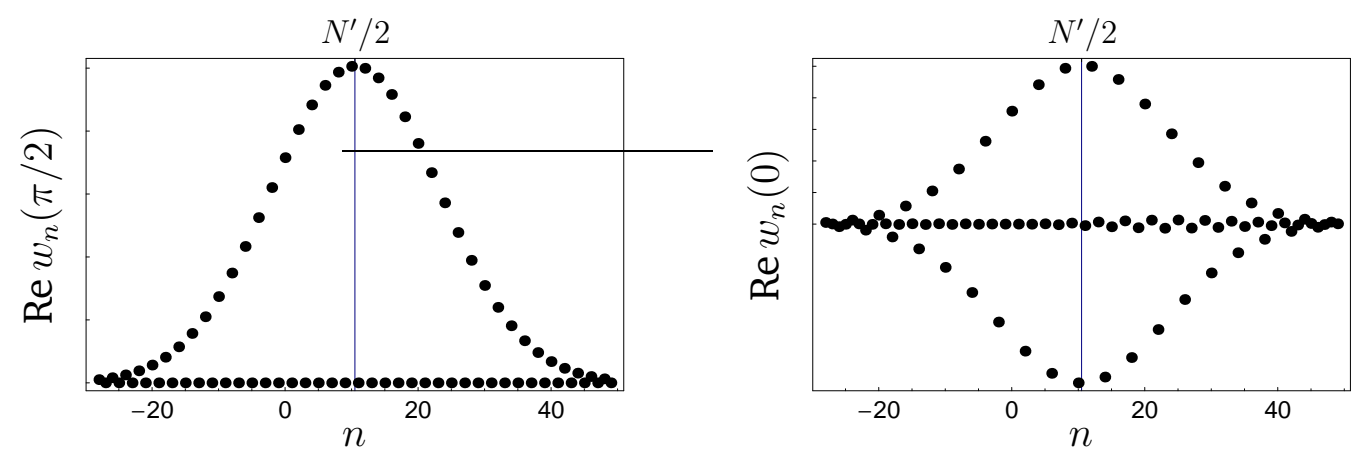

Figure 4.2: Exact weight factors $w_{n}(\varphi)$, Eq. (4.25), for $|\varphi|=\pi / 2$ (left) and $\varphi=0$ (right). Here we have chosen the modulation index $\kappa=8001 \pi / 4$ according to Eq. (4.30). Indeed, only for even values of the summation index the weight factors do not vanish. The weight factors are purely real for both choices of $\varphi$. However, for $\varphi=0$ we are dealing with weights which alternate in sign. Parameters are $N^{\prime}=21, \Delta n=12.71$.

for even values $n=2 m$ of the summation index. As a consequence the probability amplitude takes the form

$$
c_{e}=i 2 \pi \frac{\Omega_{g e}}{\Delta} e^{i \beta(t)} \exp \left(i \frac{\pi}{2} N^{\prime} \xi^{\prime}\right) \sum_{m} w_{2 m}(\varphi) \exp \left[-2 \pi i\left(m-\frac{2 m^{2}}{N^{\prime}}\right) 2 \xi^{\prime}\right] .
$$

We point out that the character of the sum in Eq. (4.33) critically depends through the weight factors $w_{n}(\varphi)$ on the particular choice of the relative phase $\varphi$. In the following we discuss this issue in more detail.

\subsubsection{Choice of phase}

Moreover, from the approximated weight factors of Eq. (4.32) we find that for the choice of $|\varphi|=\pi / 2$ and $\varphi=0$ the complex nature of the phase factor vanishes and we deal with purely real weight factors. Thus the only $m$-dependence left results from the Gaussian.

In Figure 4.2 we show an example for the exact weight factor distribution $w_{m}$, Eq. (4.25), for two choices of the relative phase and the modulation index $\kappa$, Eq. (4.30). The depicted diagrams support our approximation Eq. (4.31) since only for even values of $n$ we find non-zero contributions to the total excitation probability amplitude $c_{e}$. In both cases the weight factors are purely real. However, for $\varphi=0$ the associated weights alternate in sign. In addition, we remark that for other values of the relative phase $\varphi$ we deal with complex-valued weight factors.

In section 5.2.1 we discuss the properties of the resulting sum in the context of factorization. 


\subsubsection{Summary}

On first sight the probability amplitudes $c_{e}$ derived in this chapter and the one of Eq. (3.58) derived in chapter 3 are of the same shape. For each system the weight factor distribution involves a Gaussian. However, a more detailed comparison reveals severe differences.

In the present system the summation range in $c_{e}$ is solely determined by the Gaussian distribution of weight factors. In particular, the width $\Delta m$ determines the number of terms which effectively contribute to this sum. Due to the Gaussian distribution the weights vary as a function of the summation index and also critically depend on the relative phase $\varphi$.

This stands in contrast to the first system where the finite number of intermediate states in the harmonic manifold solely determines the truncation of the summation range. There we have realized an almost constant distribution of weight factors by independently choosing a Gaussian of large width $\Delta m$. Thus the terms of the sum appear with slowly varying weight factors.

In addition, there is another difference concerning the sign of the dimensionless argument which is proportional to the chirp $\phi^{\prime \prime}$. In the case of the Floquet ladder there is no restriction on the sign of the frequency chirp. We are free to apply positive or negative frequency sweeps. For the first system, however, it is the sequential excitation mechanism which determines the sign of the chirp. It has to be opposite to the offset $\delta_{m}$ of the intermediate state $|m\rangle$ in the harmonic manifold, that is $\operatorname{sign}\left(\phi^{\prime \prime} \delta_{m}\right)<0$.

In chapter 5.2 we show how to factor a number $N^{\prime}$ using the Gauss sum, Eq. (4.33). In particular, we investigate how the ability to factor numbers depends on the particular choice of the relative phase.

\subsection{Pulse train}

We now turn to an alternative approach for realizing a Gauss sum in a physical system. In contrast to the method of the preceding section the quadratic phase factors do not emerge due to a chirped laser pulse. In contrast, they arise from the combination of a linear time-variation of the resonance condition and a sequence which contains a limited number of short laser pulses. The excitation probability amplitude induced by this pulse sequence is the sum over contributions from individual pulses. 


\subsubsection{Excitation probability in the weak field limit}

In the present approach towards factorization of numbers with laser pulses we modulate the energy of the excited state by an electric field

$$
E_{\mathrm{m}}(t)=\mathcal{F}_{0} \frac{t}{T}
$$

with amplitude $\mathcal{F}_{0}$ and time scale $T$.

Moreover, we drive the one-photon transition with the electric field $E_{\mathrm{d}}(t)$, Eq. (4.2), consisting of a train of $M+1$ delta-shaped pulses

$$
f(t)=\frac{1}{M+1} \sum_{m=0}^{M} \delta(t-m T),
$$

where the $m$-th pulse arrives at the time $m T$. We note that the temporal width of the individual pulses has to be small compared to the separation $T$ of adjacent pulses. When we substitute the pulse train $f(t)$, Eq. (4.35), into Eq. (4.9) we arrive at

$$
c_{e}(t)=i \frac{\Omega_{g e}}{M+1} e^{i \beta(t)} \sum_{m=0}^{M} \exp \left[i\left(\delta T m-\frac{\Omega_{e e}}{2} T m^{2}\right)\right]
$$

where the phase is given by

$$
\beta(t) \equiv \frac{1}{2} \frac{\Omega_{e e}}{T} t^{2}
$$

with Rabi frequency

$$
\Omega_{e e}=\frac{\wp_{e e} \mathcal{F}_{0}}{\hbar} .
$$

Here we have assumed that the integration range is chosen long enough in order to cover the complete pulse train $f(t)$.

Again the excitation probability amplitude involves the sum over quadratic phase factors. A clear advantage of this sum bases on the fact that every phase factor enters the sum with the same weight.

\subsubsection{Purely quadratic phases for a specific choice of parame- ters}

Though in principle we could apply the same route to work out the Gauss sum character of the excitation probability amplitude as in section 4.2 we follow here a different route. The key idea of this alternative approach bases on the elimination of the phase factor which is linear in the summation index $m$. For this purpose we relate the detuning $\delta$ and the pulse separation $T$ to the integer

$$
N_{0} \equiv \frac{\delta T}{2 \pi}
$$




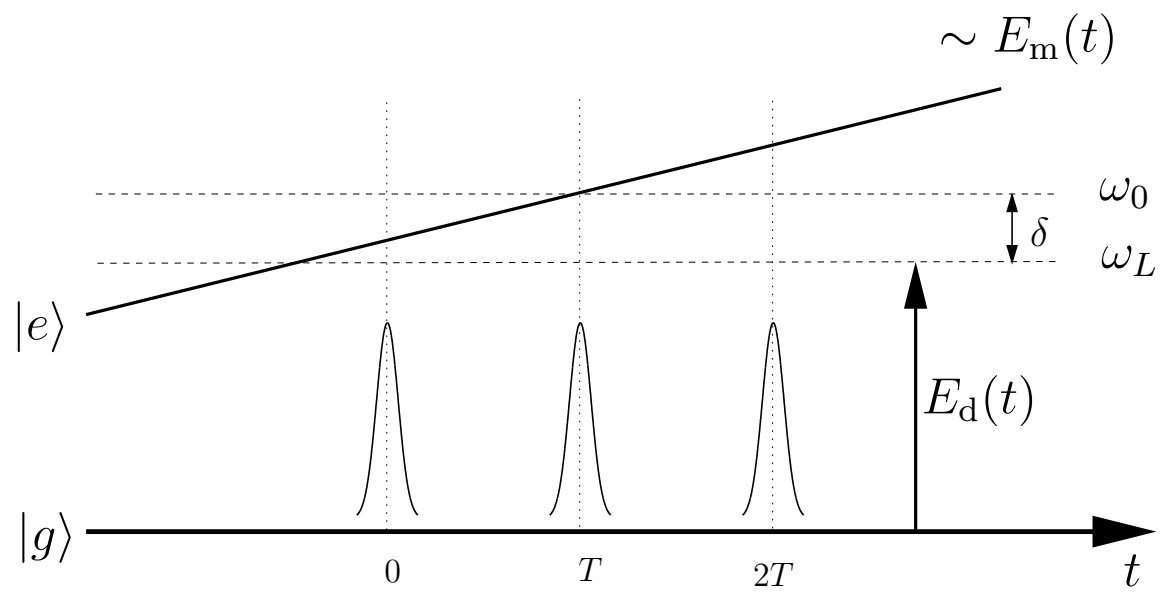

Figure 4.3: Excitation by a pulse train. We consider a two-level system with ground state $|g\rangle$ and excited state $|e\rangle$. The modulation field $E_{m}(t), E q$. (4.34), causes a linear variation of the excited state energy. Simultaneously a pulse sequence of $M+1$ delta-shaped laser pulses $E_{d}(t), E q$. (4.35), with carrier frequency $\omega_{L}=\omega_{0}-\delta$ induces transfer in the weak field limit. Consecutive pulses are separated by $T$.

Reprinted figure from W. Merkel, I. Sh. Averbukh, B. Girard, G. G. Paulus and W. P. SCHLEICH, "Factorization of numbers with physical systems", Fortschritte der Physik 54, Nos. 8-10, p. 856-865 (2006). Copyright Wiley-VCH Verlag GmbH \& Co. KGaA. Reproduced with permission.

With this choice we find

$$
-\frac{1}{2} \Omega_{e e} T m^{2} \equiv-2 \pi m^{2} \frac{N_{0}}{\xi_{0}}
$$

for the quadratic phase where we have introduced the dimensionless variable

$$
\xi_{0} \equiv \frac{2 \delta}{\Omega_{e e}} .
$$

By varying the detuning $\delta$ and/or the amplitude of the modulation field we are able to control $\xi_{0}$. As a consequence, the excitation probability reads

$$
c_{e}(t)=i \Omega_{g e} e^{i \beta(t)} \frac{1}{M+1} \sum_{m=0}^{M} \exp \left[-2 \pi i m^{2} \frac{N_{0}}{\xi_{0}}\right],
$$

where the roles of the number $N_{0}$ and the argument $\xi_{0}$ are interchanged in contrast to the previous Gauss sum $\mathcal{S}_{N}$ : Now $N_{0}$ enters the phase factor in the numerator whereas the argument $\xi_{0}$ appears in the denominator.

We remark that this result is very close to the standard Gauss sum $G(a, b)$ of number theory, see Appendix A. Here all purely quadratic phase factors appear with the same weight. However, the summation range in this truncated Gauss sum does not cover a whole period as it is determined by the number $M$ of pulses in the pulse train. 


\subsection{Discussion}

In this chapter we have investigated two examples for laser-driven one-photon transitions in two-level systems. In both approaches we assume as a major prerequisite a permanent dipole moment associated with the excited state.

A cw microwave field generates a manifold of equidistant Floquet satellites of the excited state. We calculate the excited state probability amplitude induced by a weak chirped laser pulse in first order perturbation theory.

The second approach bases on a linear variation of the resonance condition of the two-level system. When driven by a sequence of weak delta-shaped pulses we find that the excited state probability amplitude arises from the superposition of single pulse contributions.

In each approach the excited state probability amplitude contains the sum over quantum paths which are characterized by quadratic phase factors. However, both approaches differ in the origin of the quadratic phases. In the Floquet ladder they are due to the chirped pulse, whereas in the pulse train approach they originate from a linear chirp of the resonance condition. 


\section{CHAPTER 5}

\section{Factorization with physical systems}

In chapter 2 we have proposed several approaches to reveal the (prime) factors from a factorization interference pattern arising from the Gauss sum for an adequately encoded number $N$. Two schemes base on the standard Gauss sum $\mathcal{S}_{N}(\xi)$, and the third scheme makes use of another type of Gauss sum $\mathcal{A}_{N}(\xi)$. For each scheme we have introduced rules to determine the (prime) factors of $N$.

In the last chapters 3 and 4 we have analyzed three quantum optical systems which involve various types of (Gauss) sums in their excitation probability amplitudes $c_{e}$. For each system we have shown how to encode the number and the dimensionless argument in terms of parameters characterizing each physical system.

Fluorescence measurements provide direct access to Gauss sums in experiments. Here the acquired signal is proportional to the excited state population $\left|c_{e}\right|^{2}$.

In the present chapter we demonstrate the factorization property of the various excitation probability amplitudes derived in the previous chapters. For each of the three considered physical systems we provide examples basing on numerical simulations.

This chapter is organized as follows: In section 5.1 we sample the fluorescence signal $\sim\left|\mathcal{S}_{N}(\xi)\right|^{2}$ as a continuous function of the dimensionless argument $\xi$. Whenever the signal reveals a well-pronounced maximum in the vicinity of a prime argument we identify this prime number with one of the factors of $N$. Moreover, we demonstrate a remarkable scaling property of this scheme: A single experimental realization for the number $N$ allows to reveal the prime factors of another number $\widetilde{N}$.

In section 5.2 we demonstrate that the excitation probabaility amplitude, 
Eq. (4.33), arising in the chirped Floquet ladder involves a sum over quadratic phase factors only for certain values of the relative phase $\varphi$ between the modulating and the driving field. On the other hand we pursue an alternative approach which bases on the analysis of the fluorescence signal $\left|\mathcal{S}_{N}(\ell)\right|^{2}$ at integer arguments $\xi=\ell$ only.

For the second realization basing on laser-driven two-level systems we have derived in section 4.3 the excitation propability amplitude $c_{e}$. In contrast to the previous schemes $c_{e}$ is proportional to another type of Gauss sum $\mathcal{A}_{N}^{(M)}$. In section 5.3 we demonstrate the factorization property for this type of Gauss sum on the basis of discrete measurements of the signal $\left|\mathcal{A}_{N}^{(M)}(\ell)\right|$ at integer arguments. We find that this scheme is advantageous compared to the previous schemes as it requires only few terms in the sum.

Finally in section 5.4 we compare the three proposed factorization schemes and discuss differences and challenges in the context of an experimental realization.

\subsection{Chirped multistate ladder}

In chapter 3 we have derived in second order perturbation theory the excitation probability amplitude $c_{e}$, Eq. (3.58), which is proportional to the Gauss sum

$$
\mathcal{S}_{N}(\xi) \equiv \sum_{m} w_{m} \exp \left[2 \pi i\left(m+\frac{m^{2}}{N}\right) \xi\right] .
$$

In the context of factorization we neglect the prefactor and restrict our investigation solely to the Gauss sum. The exact weight factors

$$
w_{m} \equiv-\frac{d_{m}}{2} \operatorname{erfc}\left(\zeta_{m}\right) \exp \left[-\left(\frac{m+N / 2}{\Delta m}\right)^{2}\right]
$$

depend on the complementary error function with argument

$$
\zeta_{m} \equiv \frac{(m+N / 2}{\Delta m} i \sqrt{1-i a} .
$$

In Appendix E we have derived an approximation, Eq. (E.20), for $\operatorname{erfc}\left(\zeta_{m}\right)$, which takes us to the approximated weight factors, Eq. (3.65).

Moreover, as an idealization we choose the width $\Delta m$ of the weight factor distribution such that the Gaussian varies slowly as a function of the quantum number $m$. Together with the assumption of constant product of Rabi frequencies

$$
d_{m}=\text { const. }
$$

we make sure that all excitation paths are almost equally favored. 
For the aquisition of the continuous factorization interference pattern we require sufficient resolution in the dimensionless argument $\xi$ for sampling the signal in the vicinity of candidate prime numbers.

\subsubsection{Continuous sampling of the signal}

We present numerical results which are generated on the basis of an artifical ladder system which contains $D$ intermediate states. Here we adapt the dimension $D$ of the harmonic manifold to the number $N$ to be factorized. The larger the number $N$ the more intermediate states are required for achieving a well-contrasted signal. For factoring the number $N$ we require approximately $D=N \ldots 2 N$ intermediate states. Certainly, this requirement is a clear disadvantage from the viewpoint of scalability which is a major prerequisite for the factorization of large numbers. For this reason we demonstrate the factorization property only for a small number $N$.

In Figure 5.1 we depict the signal $\left|\mathcal{S}_{N}(\xi)\right|^{2}$ for $N=15$ as a function of the continuous argument $\xi$. On the left we present an overview over the whole signal. Only for negative arguments $(\xi<0)$ the excitation probability amplitude $c_{e}$ takes on the form of a Gauss sum. We recall from our previous analysis in chapter 3 that two-photon excitation via sequential quantum paths can only occur for negative values of $\xi$.

The insets in Figure 5.1 depict the signal in the vicinity of the candidate primes $-\xi=\ell=2,3,5$ and 7 . The line shows the signal generated with the exact weight factors, Eq. (3.59), whereas the dashed line depicts the signal generated with the approximated weight factors, Eq. (3.65). We remark that the latter signal agrees well with the exact results and thus allows for determining the prime factor components of $N$.

We identify pronounced maxima of the signal at the arguments $-\xi=\ell=$ 3 and 5 . In contrast, the signal does not show any peculiarities at non-factors $(-\xi=\ell=2$ and 7$)$.

Furthermore, we notice maxima which are located at non-integer values of $\xi \neq \ell$. Therefore they are not relevant in the context of factorizing the number $N$. However, such maxima do matter in the context of factoring other numbers $\widetilde{N}$. In section 5.1.2 we discuss this fact in more detail.

\subsubsection{Scaling property}

In section 2.2.2 we have found a remarkable scaling property intrinsic to the Gauss sum $\mathcal{S}_{N}$. Here we demonstrate that the excitation probability amplitude in a chirped two-photon transition indeed has the ability not only to factor the number $N$ but also another number $\widetilde{N}$. 

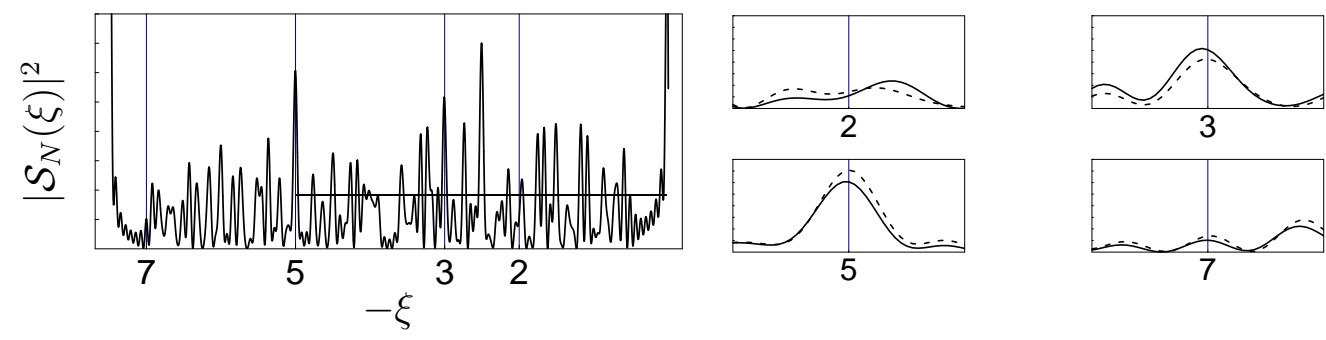

Figure 5.1: Factorization of $N=15$ by continuous scanning.

On the left we provide an overview over the complete signal $\left|\mathcal{S}_{N}(\xi)\right|^{2}$ as a function of the dimensionless chirp $\xi$. The intermediate manifold for this example covers $D=15$ states. The insets depict the magnified signal in the vicinity of candidate prime factors. We present the signal generated with exact weight factors, Eq. (3.59)(line), whereas the dashed line denotes the signal arising from the approximated weights, Eq. (3.65). The pronounced maxima at the prime factors $-\xi=3$ and 5 are clearly visible. In contrast, the signal does not show any peculiarities at the non-factors $-\xi=2$ and 7 . Here we have assumed $d_{m}=$ const. and parameters are chosen such that the width of the Gaussian is $\Delta m=50.8$

For this purpose we reconsider the signal $\left|\mathcal{S}_{N}\right|^{2}$ acquired for the number $N$ at other positions $\tilde{\xi}$ of the rescaled chirp, which are determined by the replacement rule

$$
\xi \rightarrow \frac{N}{\widetilde{N}} \tilde{\xi}
$$

In order to factorize the number $\widetilde{N}$ we have to analyze the original signal $\left|\mathcal{S}_{N}\right|^{2}$ in the vicinity of integer arguments $\tilde{\xi}=\tilde{\ell}$. In Figure 5.2 we demonstrate this property with an example. Here we factorize $\widetilde{N}=33$ basing on the original signal acquired for $N=15$. The magnified insets clearly indicate the prime factors of $\widetilde{N}$.

In the context of factorizing $\widetilde{N}$ only maxima of the signal $\left|\mathcal{S}_{N}\right|^{2}$ are relevant if they are positioned at prime arguments $\tilde{\xi}=\tilde{\ell}$ even though such maxima were meaningless the context of the factorization of $N$. In contrast, maxima corresponding to prime factors of $N$ do (in general) not play a role in the factorization of $N$.

\subsection{Factorization based on Floquet ladder}

Here we demonstrate the factorization property of the excitation probability amplitude, Eq. (4.33), derived in section 4.2 for the chirped Floquet ladder. We find that $c_{e}$ is proportional to the sum

$$
\mathcal{S}_{N^{\prime}}^{(\varphi)} \equiv \sum_{n} w_{n}(\varphi) \exp \left[2 \pi i\left(-n+\frac{n^{2}}{N^{\prime}}\right) \xi^{\prime}\right]
$$



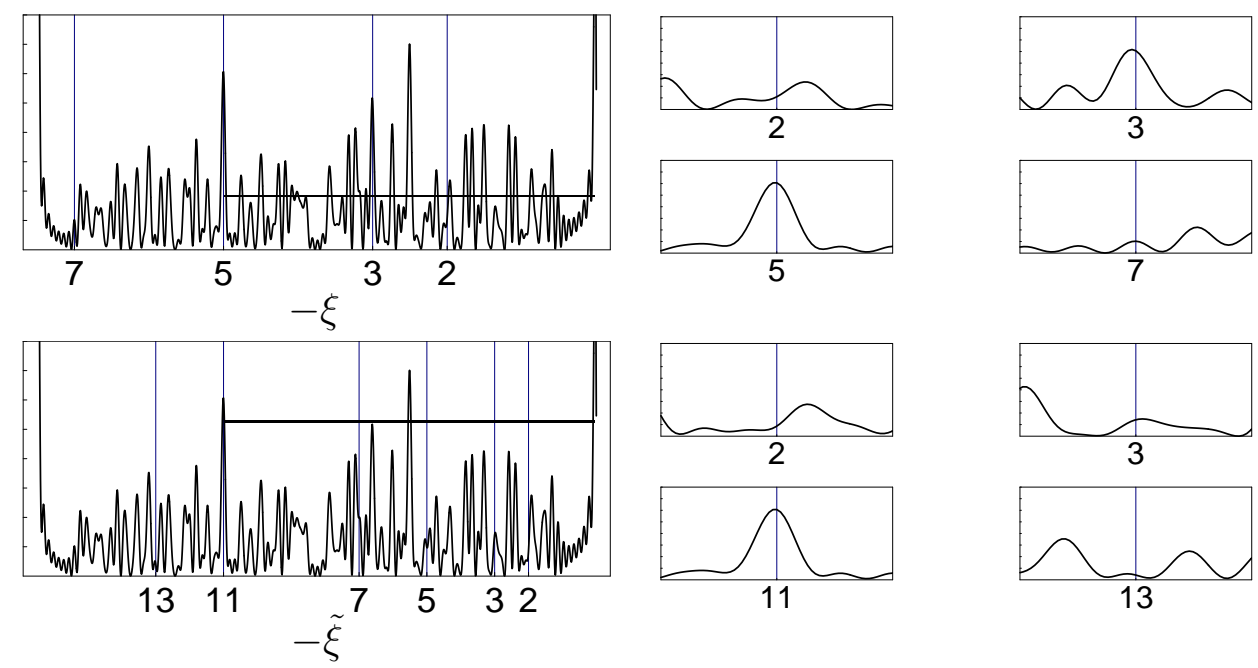

Figure 5.2: Revealing the prime factors of $\widetilde{N}=33$ by scaling $\left|\mathcal{S}_{15}\right|^{2}$.

By scaling the original signal for $N=15$ (top) we obtain the signal corresponding to $\widetilde{N}=33$ (bottom). In each case vertical lines depict the positions of candidate prime factors. From the insets we find the prime factor decomposition of $N$ and $\widetilde{N}$.

with the weight factors

$$
w_{n}(\varphi)=e^{i n \varphi} J_{n}(\kappa) g_{n}\left(N^{\prime}, \Delta n\right) \equiv p_{n}(\varphi) g_{n}\left(N^{\prime}, \Delta n\right) .
$$

Here the Gaussian distribution $g_{n}\left(N^{\prime}, \Delta n\right)$ of width $\Delta n$ is allways positive and the character of the weight factors is solely determined by

$$
p_{n}(\varphi) \equiv e^{i n \varphi} \cos \left(n \frac{\pi}{2}\right) \text {. }
$$

For the following we neglect all other prefactors since they do not affect the ability to factor numbers.

For this purpose we present two schemes. The first scheme bases on a continuous sampling of the signal $\left|c_{e}\right|^{2} \sim\left|\mathcal{S}_{N^{\prime}}^{(\varphi)}\right|$. In correspondence with our analysis in section 4.2 we find that this scheme indeed requires the choice of the relative phase $|\varphi|=\pi / 2$. In this case the weight factors are such that $c_{e}$ is proportional to a Gauss sum. In addition we discuss another case of $\varphi=0$. Here the weightfactors are also purely real but alternate in sign as a function of the summation index. It is remarkable that also this kind of sum carries alternative information on the factors of $N^{\prime}$. For completeness we also present an example for the factorization interference pattern for the case for other values of $\varphi$. Here we deal with complex-valued weight factors. Thus the phases of the individual terms contributing to the sum are not quadratic anymore.

In contrast, for the second scheme it suffices to aquire the signal $\sim\left|\mathcal{S}_{N^{\prime}}^{|\pi / 2|}\right|^{2}$ for integer values of the dimensionless chirp $\xi^{\prime}=\ell^{\prime}$ only. We show that this discrete data set impressively allows to reveal the divisors of the number $N^{\prime}$. 


\subsubsection{Factorization with different types of sums}

In chapter 4 we have found that for the Floquet ladder the excitation probability amplitude $c_{e}$ is proportional to a weighted sum $\mathcal{S}_{N^{\prime}}^{(\varphi)}$ over quadratic phase factors. In general the weightfactors $w_{n}(\varphi)$ are complex-valued and the particular choice of the relative phase $\varphi$ critically determines the nature of the resulting $\operatorname{sum} \mathcal{S}_{N^{\prime}}^{(\varphi)}$.

In Eq. (4.31) we have found the approximation

$$
J_{n}(\kappa) \sim \cos \left(n \frac{\pi}{2}\right)
$$

of the Bessel-function for the specific choice of the modulation index $\kappa$, Eq. (4.30). This relation implies that we find only non-zero terms in the sum for even values of the summation index $n$.

In the following we discuss the resulting sums for several cases of the relative phase $\varphi$.

$|\varphi|=\pi / 2$ : Gauss sum

For $|\varphi|=\pi / 2$ we find

$$
p_{n}( \pm \pi / 2)=\exp \left( \pm i n \frac{\pi}{2}\right) \cos \left(n \frac{\pi}{2}\right)=\frac{1+(-1)^{n}}{2}= \begin{cases}1 & \text { for } n \text { even } \\ 0 & \text { for } n \text { odd }\end{cases}
$$

which implies that all non-zero weight factors appear with positive signs (which is depicted in Figure 4.2). Hence, the excitation probability amplitude $c_{e}$ is indeed proportional to a Gauss sum. However, this sum slightly differs from the Gauss sum $\mathcal{S}_{N^{\prime}}$ discussed in chapter 2 due to a slight difference in sign in the phase factor. Since our factorization scheme bases on the absolute value squared of the Gauss sum this difference in sign does not manifest in the factorization interference pattern. Therefore we stick to the notation

$$
\mathcal{S}_{N^{\prime}}^{( \pm \pi / 2)}=\mathcal{S}_{N^{\prime}}
$$

and demonstrate in Figure 5.3 that the signal $\left|\mathcal{S}_{N^{\prime}}^{( \pm \pi / 2)}\right|^{2}$ indeed allows to identify the prime factors of $N^{\prime}=21$.

$\varphi=0$ : Alternating sum over quadratic phase factors

For $\varphi=0$ Eq. (5.5) reduces and only

$$
p_{n}(0)=\cos \left(n \frac{\pi}{2}\right)=\left\{\begin{aligned}
0 & \text { for } n \text { odd } \\
(-1)^{n / 2} & \text { for } n \text { even }
\end{aligned}\right.
$$



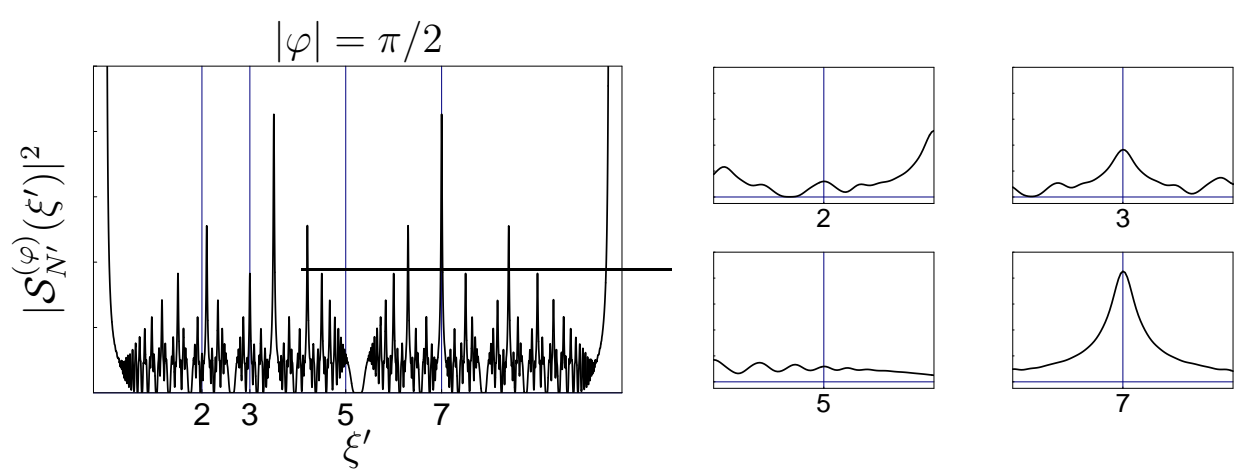

Figure 5.3: Floquet ladder: Factorization of $N^{\prime}=21$ based on the Gauss sum $\mathcal{S}_{N^{\prime}}^{( \pm \pi / 2)}$

On the left we show the complete signal $\left|\mathcal{S}_{N^{\prime}}^{( \pm \pi / 2)}\right|^{2}=\left|\mathcal{S}_{N^{\prime}}\right|^{2}$ as a function of the continuous rescaled chirp $\xi^{\prime}$. Here we have chosen the phase of the $c w$-field as $|\varphi|=\pi / 2$. On the right we show magnified regions in the vicinity of candidate prime factors. The pronounced maxima at the prime factors $\ell=3$ and 7 are clearly visible. In contrast, at $\ell=2$ and 5 the signal does not show any peculiarities. The electric field parameters were chosen so that the width of the weight factor distribution is $\Delta n=12.71$ and the summation range in the Gauss sum covers approximately 78 satellites in the Floquet ladder. Here the value of the modulation index is given by $\kappa=8001 \pi / 4$.

determines the sign of the weight factors with which the quadratic phase factors enter the sum in $c_{e}$. For even arguments $n=2 m$ we find that the real-valued weight factors $w_{2 m}(0)$ alternate in sign. In Figure 4.2 we have already seen this chracteristic property. We conclude that the resulting sum $\mathcal{S}_{N^{\prime}}^{(\varphi)}$ is not of the form of a Gauss sum because the individual terms enter the sum with alternating signs.

Nevertheless, the corresponding signal $\left|\mathcal{S}_{N^{\prime}}^{(\varphi=0)}\right|^{2}$ can also be employed for factorization since it carries information on the factors of $N^{\prime}$ in an alternative way: The signal vanishes for factors of $N^{\prime}$. In Figure 5.4 we demonstrate this finding with the continuous factorization interference pattern for $N^{\prime}=21$ and the relative phase $\varphi=0$.

\section{Other values of $\varphi$ : sum over non-quadratic phase factors}

In contrast, for other choices of the relative phase $\varphi$ the prefactor $p_{n}(\varphi)$, Eq. (5.5), is complex-valued. Thus the terms entering the sum $\mathcal{S}_{N^{\prime}}^{(\varphi)}$ have a phase dependence which differs from the purely quadratic dependence characteristic to the Gauss sum $\mathcal{S}_{N^{\prime}}$. Thus we expect that the sum $\mathcal{S}_{N^{\prime}}^{(\varphi)}$ does not have the periodic properties as the Gauss sum.

In Figure 5.5 we provide for completeness the continuous factorization interference pattern for $\varphi=\pi / 3$ and $N^{\prime}=21$. In contrast to $\left|\mathcal{S}_{N^{\prime}}^{(0)}\right|^{2}$ we observe a zero of the signal only at one of the prime factors of $N^{\prime}$. However, the sig- 

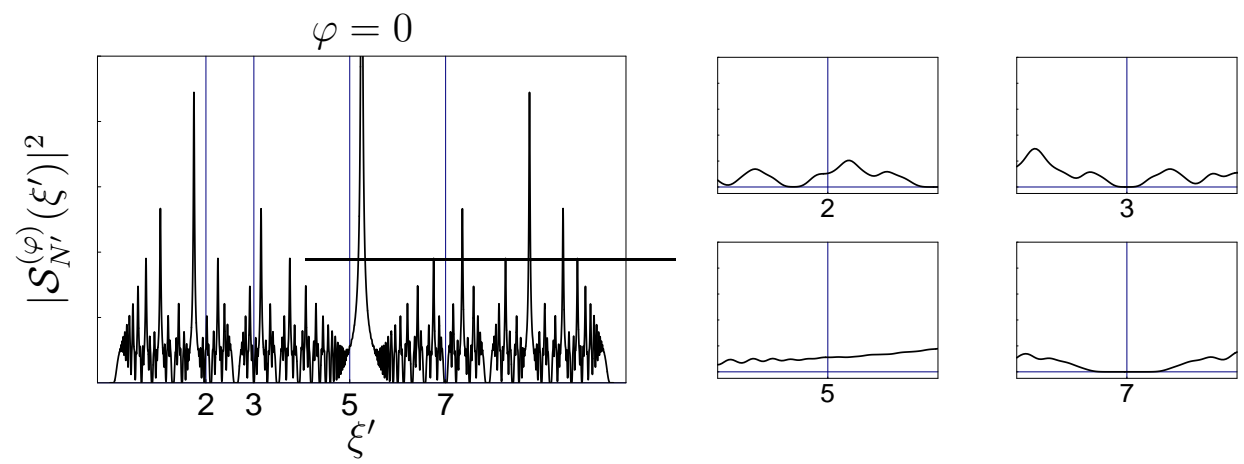

Figure 5.4: Floquet ladder: Factorization of $N^{\prime}=21$ based on the sum $\mathcal{S}_{N^{\prime}}^{(\varphi)}$ for $\varphi=0$. On the left we show the complete signal $\left|\mathcal{S}_{N^{\prime}}^{(0)}\left(\xi^{\prime}\right)\right|^{2}$ as a function of the continuous rescaled chirp $\xi^{\prime}$. On the right we show magnified regions in the vicinity of candidate prime factors. In contrast to Figure 5.3 we observe a zero signal at the prime factors $\ell=3$ and 7 whereas the signal does not show any peculiarities at $\ell=2$ and 5 . The parameters are the same as for Figure 5.3.

nal $\left|\mathcal{S}_{N^{\prime}}^{(\pi / 3)}\right|^{2}$ does not display any meaningful features at the other prime factor $\ell^{\prime}=3$ and the non-factor arguments $\ell^{\prime}=2$ and 5 .

Thus, sums $\mathcal{S}_{N^{\prime}}^{(\varphi)}$ arising from relative phases $\varphi$ different from 0 and $|\pi / 2|$ do not seem to be suited for factorization.

In the following we restrict our analysis to the specific choice $|\varphi|=\pi / 2$ for the relative phase. Here the resulting sum $\mathcal{S}_{N^{\prime}}^{( \pm \pi / 2)}$ is indeed a Gauss sum $\mathcal{S}_{N^{\prime}}$, whose properties were investigated in chapter 2 . We pursue an alternative approach to factorization, which bases on discrete sampling of the signal at integer arguments.

\subsubsection{Fluorescence signal at discrete arguments}

In our previous approaches to factorization we have relied on continuous measurements of the signal $\left|\mathcal{S}_{N^{\prime}}\right|^{2}$. Here we follow an alternative way to factorize $N^{\prime}$. The present approach bases on discrete measurements of the signal $\left|\mathcal{S}_{N^{\prime}}\right|^{2}$ at integer arguments only. However, we require for this purpose sufficient control over the rescaled chirp $\xi^{\prime}$ so that we can precisely tune it to integer values $\xi^{\prime}=\ell^{\prime}$ only.

When we consider the phase factor in the Gauss sum, Eq. (5.3), we find that the term linear in the summation index drops out. In this case the probability amplitude $c_{e}$ is proportional to the Gauss sum

$$
\mathcal{S}_{N^{\prime}}\left(\ell^{\prime}\right)=\sum_{n} w_{n}\left(\frac{\pi}{2}\right) \exp \left(2 \pi i n^{2} \frac{\ell^{\prime}}{N^{\prime}}\right)
$$



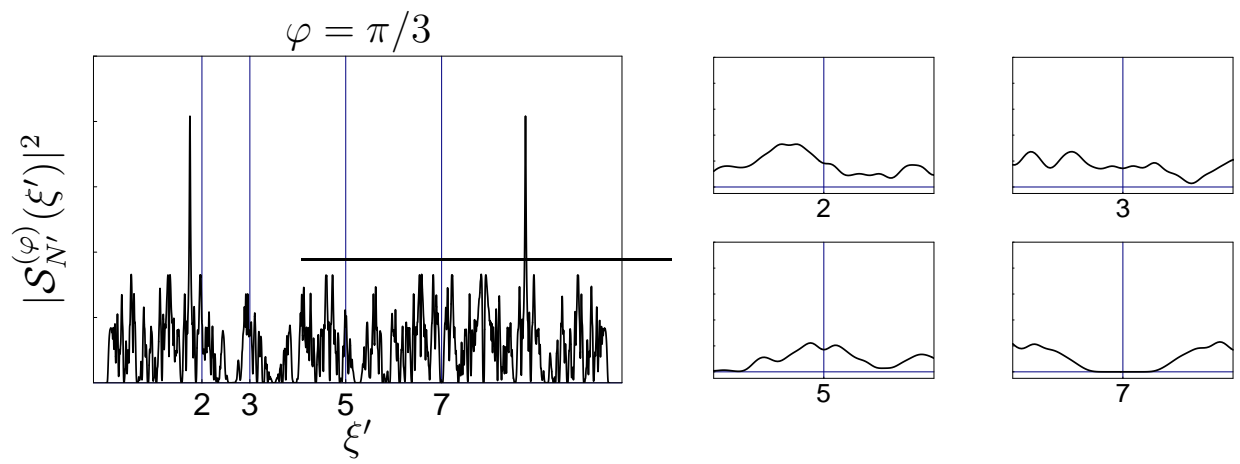

Figure 5.5: Floquet ladder: Factorization of $N^{\prime}=21$ based on the $\operatorname{sum} \mathcal{S}_{N^{\prime}}^{(\varphi)}$ for $\varphi=\pi / 3$ ? On the left we show the complete signal $\left|\mathcal{S}_{N^{\prime}}^{(\pi / 3)}\left(\xi^{\prime}\right)\right|^{2}$ as a function of the continuous rescaled chirp $\xi^{\prime}$. On the right we show magnified regions in the vicinity of candidate prime factors. We also observe a vanishing signal at the prime factor $\ell^{\prime}=7$. At the other factor $\ell^{\prime}=3$ and also at the non-factors $\ell^{\prime}=2$ and $\ell^{\prime}=5$ the signal does not show any peculiarities. The parameters are the same as in Figure 5.3.

which involves purely quadratic phases. ${ }^{1}$

In Appendix B we show that for a Gaussian weight factor distribution $w_{n}(\pi / 2)$ with large $\Delta n$ the Gauss sum $\mathcal{S}_{N^{\prime}}\left(\ell^{\prime}\right)$ is identical to the standard Gauss sum $G\left(\ell^{\prime}, N^{\prime}\right)$. In section 2.3 we have analyzed in detail the factorizing property of the standard Gauss sum $G\left(\ell^{\prime}, N^{\prime}\right)$.

In Figure 5.6 we present numerical results for the factorization of $N^{\prime}=105$ employing a truncated Floquet ladder of 306 harmonics. The signal is characterized by enhanced values at arguments corresponding to divisors of $N^{\prime}$. On the other hand the signal is suppressed at non-factor arguments. Moreover, data points with $\ell^{\prime}$ being a factor are arranged on a straight line through the origin. In addition, all data points corresponding to integer multiples of a factor of $N^{\prime}$ are characterized by the same value of the signal.

\subsection{Factorization with pulse train}

In section 4.3 we have shown an alternative system how to realize an excitation probability amplitude of the form of a Gauss sum in a laser driven two-level system. This scheme abstains from chirped pulses. Here it is the linear variation of the excited state energy which brings quadratic phases into play. The desired discrete values of the phase are selected by the individual pulses of the sequence of short pulses which drives the one-photon transition.

The resulting excitation probability amplitude $c_{e}$, Eq. (4.42), is proportional

\footnotetext{
${ }^{1}$ The autocorrelation function of the two-dimensional quantum rotor also involves this type of Gauss sum[Mac02b, Mer06].
} 


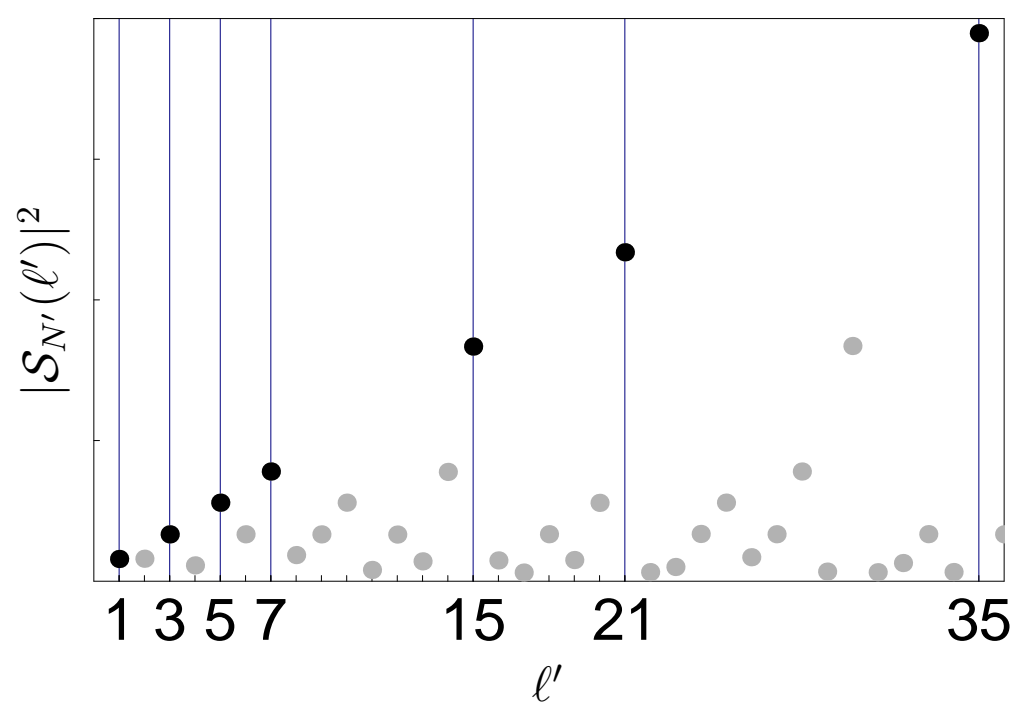

Figure 5.6: Factorization of $N^{\prime}=105$ with the Floquet ladder.

We depict the signal $\left|\mathcal{S}_{N^{\prime}}\left(\ell^{\prime}\right)\right|^{2}$, Eq. (5.10), for integer values $\ell^{\prime}$ of the rescaled chirp. At divisors of $N^{\prime}$ the signal displays enhanced values (black dots). At non-factors the signal is suppressed (gray dots). Data points corresponding to factors of $N^{\prime}$ are situated on a straight line through the origin. All data points corresponding to integer multiples of a factor are characterized by the same signal value. Here we have chosen the parameters $\Delta n=50.83$ and $\kappa=10000$ in order to realize a broad weight factor distribution. As a consequence, the summation in the Gauss sum covers $N^{\prime}<306$ harmonics of the Floquet ladder.

Reprinted figure from W. Merkel, I. Sh. Averbukh, B. Girard, G. G. Paulus and W. P. SCHLEICH, "Factorization of numbers with physical systems", Fortschritte der Physik 54, Nos. 8-10, p. 856-865 (2006). Copyright Wiley-VCH Verlag GmbH \& Co. KGaA. Reproduced with permission. 
to the normalized and truncated Gauss sum

$$
\mathcal{A}_{N_{0}}^{(M)}\left(\xi_{0}\right)=\frac{1}{M+1} \sum_{m=0}^{M} \exp \left[-2 \pi i m^{2} \frac{N_{0}}{\xi_{0}}\right]
$$

which differs from the first type of Gauss sum $\mathcal{S}_{N}$. Here the upper bound $M$ of the summation is directly related to the number of pulses in the pulse train, Eq. (4.35).

So far we have not specified the dimensionless argument $\xi_{0}=2 \delta / \Omega_{e e}$. Since the Rabi frequency $\Omega_{e e}$ is a free parameter, we have the freedom to sample the signal only at integer arguments $\xi_{0}=\ell_{0}$. Thus we obtain a discrete factorization interference pattern. For arguments corresponding to divisors of $N_{0}$ we find the same maximal value of 1 . In contrast the signal is suppressed for non-factor arguments.

In Figure 5.7 we exemplify this read-out formalism for the number $N_{0}=$ 1911. Whenever the integer argument $\ell_{0}$ is a factor of $N_{0}$ the signal $\left|\mathcal{A}_{N_{0}}^{(M)}\right|$ takes on the maximum value of unity. In contrast, at non-factor arguments the signal is suppressed.

In section 2.4.2 we have provided a detailed study of the properties of this truncated Gauss sum $\mathcal{A}_{N_{0}}^{(M)}$.

\subsection{Summary}

We devote this section to a summary and discussion of the factorization schemes introduced in sections 5.1, 5.2 and 5.3. We have provided three schemes to find the (prime) factors of a given number based on two types of Gauss sums. The first two schemes base on $\mathcal{S}_{N}$ whereas the last one bases on $\mathcal{A}_{N_{0}}^{(M)}$.

For each scheme we have presented numerical examples which demonstrate the ability to factorize numbers.

The chirped two-photon transition in a multistate ladder involves the Gauss sum $\mathcal{S}_{N}$ in the excitation probability amplitude. A continuous measurement of the signal $\left|\mathcal{S}_{N}\right|^{2}$ allows to factor relatively small numbers. Nevertheless, this type of Gauss sum is remarkable for its intrinsic scaling property which allows to reveal the factors of other numbers $\widetilde{N}$ based on one single experimental realization for the number $N$.

In the Floquet ladder approach the signal $\left|\mathcal{S}_{N^{\prime}}^{(\varphi)}\right|^{2}$ is acquired as a function of the continuous rescaled chirp $\xi^{\prime}$. For particular choices of the relative phase $\varphi$ the signal $\left|\mathcal{S}_{N^{\prime}}^{(\varphi)}\right|^{2}$ allows us to factor numbers. An alternative read-out scheme bases on discrete measurements of the signal for integer arguments. For divisors of $N$ we observe enhanced values of the signal $\left|\mathcal{S}_{N^{\prime}}^{( \pm \pi / 2)}\right|^{2}$. Moreover, datapoints corresponding to factors of $N^{\prime}$ are aligned on a straight line through the origin. 


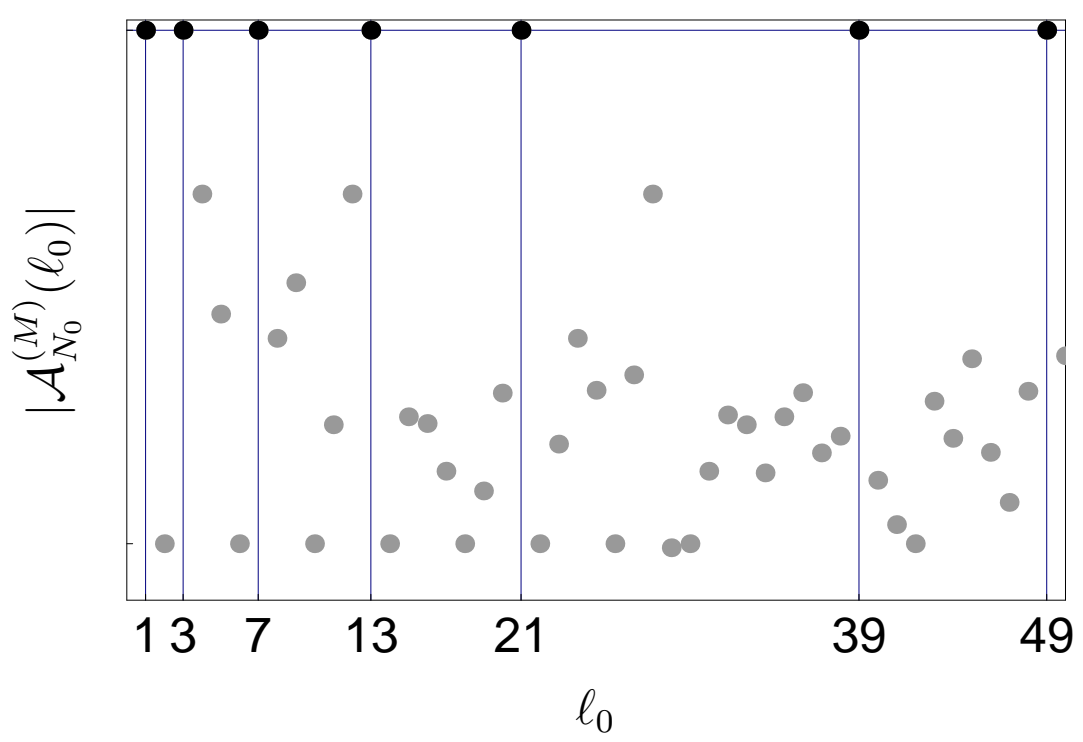

Figure 5.7: Factorization of $N_{0}=1911$ with a pulse train.

The absolute value of the truncated Gauss sum $\mathcal{A}_{N_{0}}^{(M)}$, Eq. (5.11), allows to distinguish factors from non-factors: The signal exhibits clear maxima (black dots) at divisors of $N_{0}$ whereas at non-factor arguments the signal is suppressed (gray dots). Here the upper bound $M$ of the summation in the truncated Gauss sum $\mathcal{A}_{N_{0}}^{(M)}$ is chosen as $M=10$.

Reprinted figure from W. Merkel, I. Sh. Averbukh, B. Girard, G. G. Paulus and W. P. SCHLEICH, "Factorization of numbers with physical systems", Fortschritte der Physik 54, Nos. 8-10, p. 856-865 (2006). Copyright Wiley-VCH Verlag GmbH \& Co. KGaA. Reproduced with permission. 
For the pulse train approach the proposed scheme also bases on discrete measurements of the signal $\left|\mathcal{A}_{N_{0}}^{(M)}\right|$ at integer arguments $\xi_{0}=\ell_{0}$. Already few terms in the truncated Gauss sum $\mathcal{A}_{N_{0}}^{(M)}$ allow to distinguish factors from nonfactors.

Finally we address questions concerning the experimental realization of the proposed factorization schemes.

For each of the schemes the Gauss sum is accessible by measurements of the fluorescence signal of the excited state for arguments out of the interval $[0, \sqrt{N}]$. In each case the excitation probability is proportional to the signal $\left|\mathcal{S}_{N}\right|^{2},\left|\mathcal{S}_{N}^{(\varphi)}\right|^{2}$, and $\left|\mathcal{A}_{N}^{(M)}\right|$ associated with each type of Gauss sum.

When we compare the number of measurements certainly the discrete schemes are advantageous as the signal has to be acquired only for few arguments in comparison to the continuous cases. However, both discrete approaches require precise control of the argument. Also the continuous scheme requires sufficiently good resolution in order to resolve the signal in the vicinity of candidate prime factors.

One may wonder whether the discrete scheme derived for the pulse train approach in section 4.3.2 is also applicable to the Floquet ladder system. We remind ourselves that the key idea relies on the particular choice of parameters which enables a vanishing linear dependence in the phase factor.

In the pulse train approach we dispose over three parameters, two of which are required to encode $N_{0}$ and one free parameter that allows to control the argument $\ell_{0}$.

Also in the Floquet ladder we dispose over three parameters $\delta, \Delta$ and $\phi^{\prime \prime}$ for encoding $N^{\prime}$ and $\xi^{\prime}$. Here, however, the three parameters enter the phase factor in a different combination such that we would need all three parameters to express $N^{\prime}$. Hence, there would be no parameter left for controlling the argument $\xi^{\prime}$.

It is also interesting to compare the number of terms contributing to the Gauss sums in each scheme. We find that due to the smaller period $\ell$ (with $\ell<\sqrt{N}<N)$ the second type of Gauss sum $\mathcal{A}_{N}^{(M)}$ is advantageous compared to $\mathcal{S}_{N}$ where the period is determined by $N$. In the first case already few terms allow to distinguish factors from non-factors. Yet another advantage over the schemes basing on $\mathcal{S}_{N}$ relies on the fact that all terms enter the sum $\mathcal{A}_{N}^{(M)}$ with uniform weight.

We conclude that the pulse train approach and the implementation of the Gauss sum $\mathcal{A}_{N}^{(M)}$ offers itself as the most favorable system for an experimental realization. Recently, Prof. Mehring (Universität Stuttgart) translated this scheme into the language of NMR, which led to the first experimental demonstration of factorization based on Gauss sums [Meh07]. We discuss the key ideas of this experimental realization in chapter 6 . 


\section{CHAPTER 6}

\section{NMR-Realizations of Gauss sums}

Nuclear magnetic resonance (NMR) is probably one of the most important spectroscopic techniques, which has wide-spread applications in many branches of modern science. A whole variety of precise pulse sequences allows to control the spin system and compensate for imperfections and artefacts. Moreover, another advantage consists in the high sophistication of commercially available spectrometers, which perform close to the theoretical limits.

For these reasons this powerful technique was early identified as a candidate setting to implement basic principles of quantum computing [Van05]. Already in 2001 the first demonstration of the famous Shor factorization algorithm was implemented for $N=15$ [Van01]. However, this simple exercise was quite elaborate from the experimental viewpoint when we recall that the underlying pulse sequence consisted of a few hundred RF-pulses.

In the present chapter we discuss a novel NMR-realization of the factorization problem which bases on Gauss sums. This approach allows to factor relatively large numbers on the basis of a simple pulse sequence which contains only few RF-pulses [Meh07]. Unlike the Shor algorithm our scheme does not feature an exponential speed-up in the required operations. Despite this drawback we believe that this alternative and less ambitious route offers new insight, which could ignite other interesting modifications.

In this spirit two experiments have been performed in order to demonstrate the ability of Gauss sums to factor numbers. Both realizations are modifications of the truncated Gauss sum $\mathcal{A}_{N}^{(M)}$ emerging in the pulsetrain approach of section 4.3. In section 6.1 we present the basic ideas of the experiment performed by Michael Mehring at the University of Stuttgart. We briefly review in section 6.2 an alternative experiment performed by Dieter Suter at the University of Dortmund. 


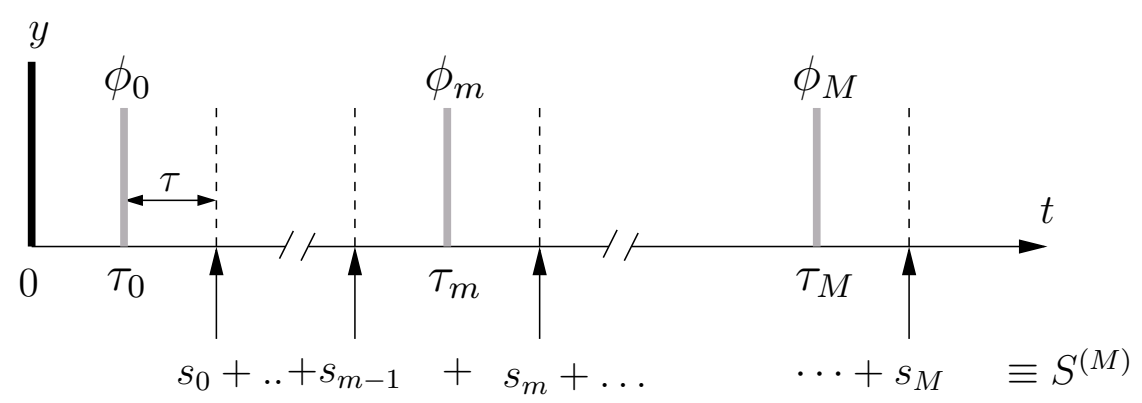

Figure 6.1: NMR implementation of a factorization scheme using Gauss sums.

The y-pulse, which prepares the initial density matrix $\rho_{\mathrm{in}}$ is followed after a time $\tau$ by a pulse which imprints the phase $\phi_{0}$ on the spins. This pulse is the first of a sequence of $M+1$ pulses each imprinting a different phase $\phi_{m}$. The duration of a complete pulse sequence with $M$ pulses is given by $M t_{c}=2 \tau=100 \mu$ s wich has to be compared with the relaxation time $T_{2}=200 \mathrm{~ms}$. At times $\tau_{m}+\tau \equiv(2 m+1) \tau+\tau$ we measure the echo, that is the polarization $s_{m}$ in $x$-direction, and sum the echos $s_{m}$ over all $m$ to obtain $S^{(M)}$. In order to implement a Gauss sum the phases $\phi_{m}$ need to be proportional to the number $N$ to be factored and have to increase linearly as a function of $m$ since the spin dynamics expressed by $s_{m}$ depends on the sum over all phases of the previous pulses.

This figure is adapted from M. Mehring, K. Müller, I. Sh. Averbukh, W. Merkel, and W. P. SChleICH, Physical Review Letters 98, p. 120502 (2007). Copyright 2007 by the American Physical Society.

\subsection{NMR experiment factors $N=157573$}

In the experiment $\mathrm{H}_{2} \mathrm{O}$ serves as an ensemble of protons with nuclear spin $1 / 2$ in Boltzmann equilibrium at room temperature. This ensemble of spins is described by a density operator

$$
\rho_{B} \equiv\left(\begin{array}{cc}
\rho_{a a} & \rho_{a b} \\
\rho_{b a} & \rho_{b b}
\end{array}\right) \equiv \frac{1}{2}\left(\begin{array}{cc}
1-\epsilon & 0 \\
0 & 1+\epsilon
\end{array}\right)
$$

where $0<\epsilon \ll 1$ denotes the population difference.

Radio frequency pulses are applied at the Larmor frequency $400 \mathrm{MHz}$ of the protons. Experiments have been performed with a Varian Infinity Plus NMR spectrometer applying a cycle time of $t_{c}=2 \tau=100 \mu \mathrm{s}$ and with relaxation time $T_{2} \approx 200 \mathrm{~ms}$. The pulse sequence shown in Figure 6.1 is based on the Carr, Purcell, Meiboom and Gill (CPMG)-sequence [Car54, Mei58] which was proposed for measuring $T_{2}$ relaxation times in inhomogeneous fields.

For the factorization experiment this sequence is modified such that the resulting evolution of the proton spins expressed by a sequence of echos which leads to the desired Gaussian-type sum.

The pulse sequence starts with an initiating $\pi / 2$-pulse in $y$-direction, which 
creates the initial density matrix

$$
\rho_{\text {in }} \equiv \frac{1}{2}\left(\begin{array}{cc}
1 & -\epsilon \\
-\epsilon & 1
\end{array}\right) .
$$

This pulse is followed by a series of $\pi$-pulses, which act at times $\tau_{k} \equiv(2 k+1) \tau$ and are individually phase shifted with respect to the $x$-axis of the rotating frame by an angle $\phi_{k}$ [War80, Ern87].

Within the rotating wave approximation and in the co-rotating frame the Bloch equations [Sar74]

$$
\frac{d}{d t}\left(\begin{array}{c}
U \\
V \\
W
\end{array}\right)=\left(\begin{array}{ccc}
-1 / T_{2} & -\Delta \omega & \operatorname{Im} \Omega(t) \\
\Delta \omega & -1 / T_{2} & \operatorname{Re} \Omega(t) \\
-\operatorname{Im} \Omega(t) & -\operatorname{Re} \Omega(t) & -1 / T_{1}
\end{array}\right)\left(\begin{array}{c}
U \\
V \\
W
\end{array}\right)
$$

determine the Bloch vector

$$
R(t) \equiv\left(\begin{array}{c}
U(t) \\
V(t) \\
W(t)
\end{array}\right)
$$

consisting of the real part

$$
U \equiv \rho_{a b} \mathrm{e}^{i \omega t}+\text { c.c. }
$$

the imaginary part

$$
V \equiv i \rho_{a b} \mathrm{e}^{i \omega t}+\text { c.c. }
$$

of the polarization and the inversion

$$
W \equiv \rho_{a a}-\rho_{b b} .
$$

Here $\Delta \omega=\omega_{0}-\omega$ and $T_{1}$ denote the detuning between the frequencies $\omega_{0}$ and $\omega$ of the transition and the driving field and the longitudinal relaxation time, respectively. Moreover, the pulse sequence translates into the time-dependent Rabi-frequency

$$
\Omega(t) \equiv \pi \sum_{k=0}^{M} \delta\left(t-\tau_{k}\right) \exp \left(i \phi_{k}\right)
$$

The initial condition, Eq. (6.2), takes the form

$$
R(0)=-2 \epsilon\left(\begin{array}{l}
1 \\
0 \\
0
\end{array}\right)
$$

We solve Eq. (6.3) by noting that during the free time evolution $\Omega(t)=0$ and hence the Bloch vector $R(t)$ evolves according to matrix

$$
A=\left(\begin{array}{ccc}
-1 / T_{2} & -\Delta \omega & 0 \\
\Delta \omega & -1 / T_{2} & 0 \\
0 & 0 & -1 / T_{1}
\end{array}\right)
$$


in time. On the other hand, when the $\delta$-pulses act we can neglect the contributions due to the free time evolution and at times $\tau_{k}$ we deal with the matrix

$$
B\left(\tau_{k}\right)=\pi\left(\begin{array}{ccc}
0 & 0 & \sin \phi_{k} \\
0 & 0 & \cos \phi_{k} \\
-\sin \phi_{k} & -\cos \phi_{k} & 0
\end{array}\right)
$$

which accounts for the phase kicks imposed by the RF-pulses. Moreover, we have made use of the relation

$$
\int_{\tau_{k}-\epsilon}^{\tau_{k}+\epsilon} d t \Omega(t)=\pi \exp \left(i \phi_{k}\right)
$$

As a consequence we find the time-evolved Bloch vector

$$
R\left(\tau_{m}+\tau\right)=\prod_{k=0}^{m} T\left(\phi_{k}\right) R(0)
$$

where the transfer matrix

$$
T\left(\phi_{k}\right) \equiv e^{A \tau} e^{B\left(\tau_{k}\right)} e^{A \tau}=\left(\begin{array}{ccc}
\mathrm{e}^{-2 \tau / T_{2}} \cos 2 \phi_{k} & -\mathrm{e}^{-2 \tau / T_{2}} \sin 2 \phi_{k} & 0 \\
-\mathrm{e}^{-2 \tau / T_{2}} \sin 2 \phi_{k} & -\mathrm{e}^{-2 \tau / T_{2}} \cos 2 \phi_{k} & 0 \\
0 & 0 & \mathrm{e}^{-2 \tau / T_{1}}
\end{array}\right)
$$

reflects the three stages of the $k$-th cycle in the pulse sequence depicted in Figure 6.1:

(i) Free time evolution for the time $\tau$ followed by

(ii) a $\pi$-pulse which imprints the phase $\phi_{k}$, and

(iii) another free time evolution stage for the time $\tau$.

As a consequence the free time evolution manifests only in the decaying exponential factors. The transverse magnetization

$$
s_{m} \equiv-\frac{1}{(2 \epsilon)} U\left(\tau_{m}+\tau\right)
$$

measured after the $m$-th cycle in the co-rotating frame reads

$$
s_{m}=\exp \left(-m \frac{2 \tau}{T_{2}}\right) \cos \left(2 \sum_{k=0}^{m}(-1)^{k} \phi_{k}\right) \text {. }
$$


Hence, the spin dynamics expressed by the signal $s_{m}$ depends on its complete history, that is the phases of all previous pulses. In particular, they enter as an alternating sum. The sum over all signals $s_{m}$ yields

$$
S^{(M)}=\sum_{m=0}^{M} \exp \left(-m \frac{2 \tau}{T_{2}}\right) \cos \left(\sum_{k=0}^{m}(-1)^{k} 2 \phi_{k}\right) .
$$

In order to achieve a quadratic dependence in the cosines of the sum in $S^{(M)}$ we have to choose the phases $\phi_{k}$ in order to satisfy the condition

$$
\sum_{k=0}^{m}(-1)^{k} \phi_{k} \stackrel{!}{=} 2 \pi m^{2} \frac{N}{\ell}
$$

In this context we make use of the well-known summation relation

$$
\sum_{k=0}^{m} k=\frac{1}{2} m(m+1)
$$

employed by the young Carl Friedrich Gauss in order to solve the exercise of adding up all integers from 1 to 100 [Der03]. Indeed, the particular choice of phases

$$
\phi_{k}=\left\{\begin{array}{cl}
(-1)^{k}(2 k-1) \pi \frac{N}{\ell} & \text { for } k \geq 1 \\
0 & \text { for } k=0
\end{array}\right.
$$

allows to achieve a sum in $S^{(M)}$, which resembles to the truncated Gauss sum $\mathcal{A}_{N}^{(M)}$. The normalized sum $\overline{\mathcal{C}}_{N}^{(M)} \equiv S^{(M)} /(M+1)$ takes the form

$$
\overline{\mathcal{C}}_{N}^{(M)}(\ell)=\frac{1}{M+1} \sum_{m=0}^{M} \exp \left(-m \frac{2 \tau}{T_{2}}\right) \cos \left(2 \pi m^{2} \frac{N}{\ell}\right) .
$$

Indeed, when the duration of the pulse sequence $2 M \tau$ is short compared to the relaxation time $T_{2}$, this sum reduces to the real part of $\mathcal{A}_{N}^{(M)}$.

In Figure 6.2 we display the results of the NMR-experiment for the factorization $N=157573$. On the left we show the time evolution of the spin under the influence of the pulse sequence presented in Figure 6.1. As a measure of the dynamics we show the echo signal $s_{m}$ following from Eq. (6.16). For factors of $N$ such as $\ell=17$ the signal is almost constant. Consequently, we find for the average $\overline{\mathcal{C}}_{N}^{(M)}(\ell=17)$ a value close to one as indicated in the bottom. However, for non-factors such as $\ell=18$ the echo signal oscillates and leads to a rather small average value $\overline{\mathcal{C}}_{N}^{(M)}(\ell=18)$, shown by the arrow. We emphasize that due to the quasi-random interference of Gauss sums $M=11$ terms are sufficient to discriminate factors from non-factors. 

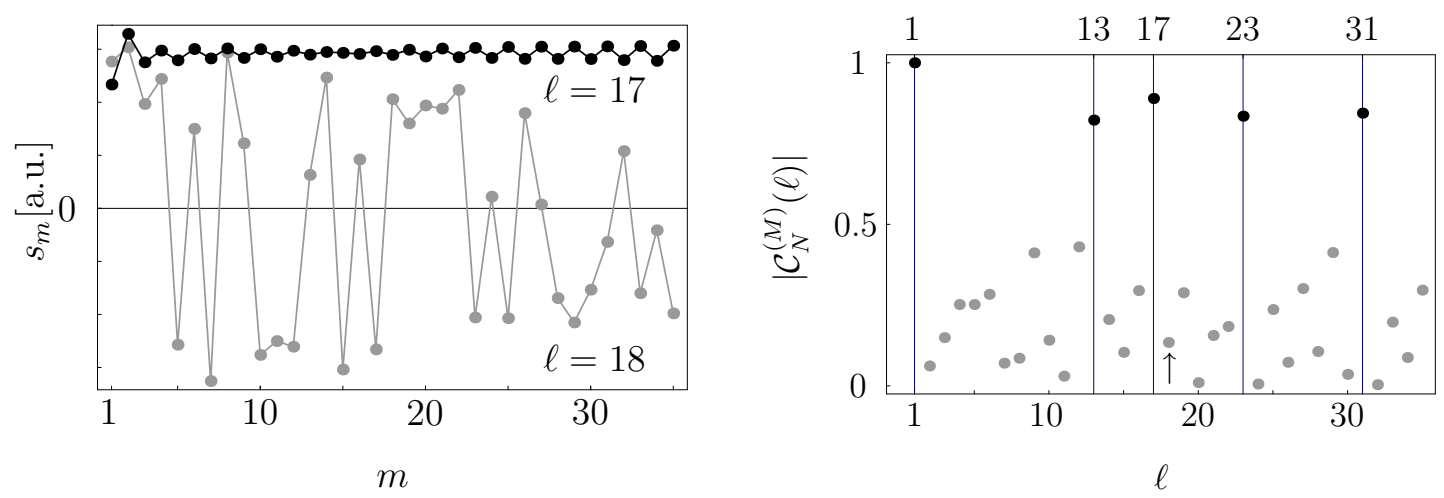

Figure 6.2: Experimental factorization of $N=157573=13 \cdot 17 \cdot 23 \cdot 31$ based on the NMR implementation of the Gauss sum $\overline{\mathcal{C}}_{N}^{(M)}$ of Eq. (6.21).

On the left we depict the echo height $s_{m}$ measured at times $\tau_{m}+\tau$ and on the right we show the resulting average $\overline{\mathcal{C}}_{N}^{(M)}$ for different arguments $\ell$. For factors such as $\ell=17$ the magnetization signals $s_{m}$ are approximately constant as a function of $m$ with an average value $\overline{\mathcal{C}}_{N}^{(M)}(17)$ close to unity. In contrast, for a non-factor such as $\ell=18 s_{m}$ oscillates around zero and when summed over $m$ almost averages out as indicated by the arrow.

This figure is adapted from M. Mehring, K. Müller, I. Sh. Averbukh, W. Merkel, and W. P. SChleICH, Physical Review Letters 98, p. 120502 (2007). Copyright 2007 by the American Physical Society.

We note that even for an appreciable decay, that is for $2 M \tau / T_{2}=2$ at the end of the sequence the factorization interference pattern looks almost identical to the one without decoherence, except for the reduced scale of the signal $\left|\overline{\mathcal{C}}_{N}^{(M)}\right|$. This result is surprising since the signal $s_{M}$ at the end of the sequence has decayed to $13.5 \%$ of its initial value. Hence, decoherence does not spoil the ability to distinguish factors from non-factors.

Moreover, we claim that extensions to larger numbers are readily possible since our method capitalizes on the quasi-randomness of the phases of Gauss sums. In Figure 6.3 we provide the factorization interference pattern of the 24-digit number

$$
N=1062885837863046188098307
$$

resulting from the Gauss sum $\mathcal{A}_{N}^{M}$, Eq. (2.41), where we choose $M=200$ as the upper limit in the summation range. Indeed, the factor $p=790645490053$ is clearly visible.

Figure. 6.3 also draws attention to the intriguing phenomenon of ghost factors: We may misinterpret datapoints with $1 / \sqrt{2}<\left|\mathcal{A}_{N}^{(M)}\right|<1$ as a factor of $N$ even though the associated argument is not a factor. We find an example for such a ghost factor at $\ell_{g}=790645394634$ with associated signal value $\left|\mathcal{A}_{N}^{(M)}\left(\ell_{g}\right)\right|=0.997645<1$.

In section 2.4.2 we have already discussed the problem of ghost factors. Moreover, we have shown that $M_{0} \sim \sqrt[4]{N}$ of terms in the Gauss sum allow to 


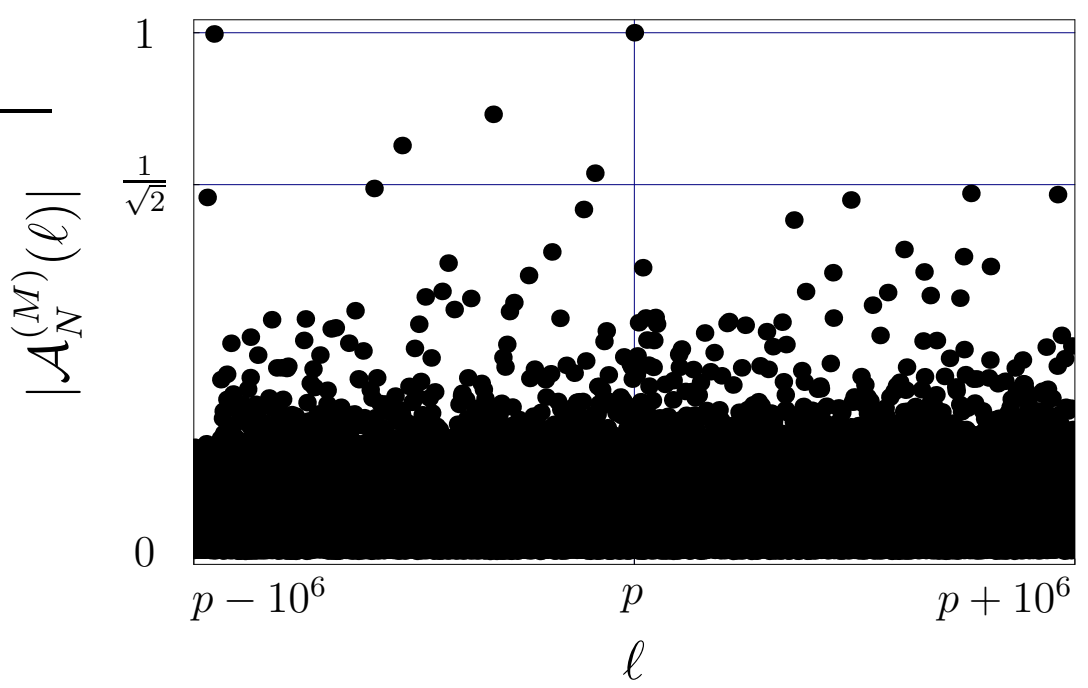

Figure 6.3: Probing the limits of the Gauss sum factorization scheme.

Factorization interference pattern for the 24-digit number $N=$ $1062885837863046188098307=p \cdot q$ obtained from a numerical simulation of the Gauss sum $\mathcal{A}_{N}^{(M)}$, Eq. (2.41), in the immediate neighborhood of the prime factor $p=790645490053$ for the truncation parameter $M=200$. Here we encounter several ghost factors with signal values out of the critical interval $(1 / \sqrt{2}, 1)$.

Reprinted figure with permission from M. MEHring, K. MÜLler, I. SH. AverbukH, W. MERKEL, and W. P. SCHLEICH, Physical Review Letters 98, p. 120502 (2007). Copyright 2007 by the American Physical Society. 
suppress all ghost factors below the natural threshold of $1 / \sqrt{2}$.

This straightforward experiment impressively demonstrates the potential of the truncated Gauss sum $\mathcal{A}_{N}^{(M)}$ in the context of factorization. Although this scheme scales exponentially it profits from the truncated summation range which allows for sufficient contrast to distinguish factors from non-factors.

\subsection{A second NMR experiment}

Recently, Dieter Suter (Universität Dortmund) has performed a different but related factorization experiment on the basis of Gauss sums [Mah07]. The starting point is again an ensemble of non-interacting spins at room temperature. These spins are driven by a pulse sequence of narrow RF-pulses where a pulse shaper allows to precisely adjust the phase

$$
\phi_{m}=2 \pi m^{2} \frac{N}{\ell}
$$

of the $m$-th pulse in the sequence depicted in Figure 6.4. Like the previous implementation this experiment requires a sequence of $m=1 . . M$ pulses for each data point. In contrast to the implementation of section 6.1 the transverse magnetization accumulates during the evolution of the complete sequence so that a single measurement following the $M$-th pulse directly yields the accumulated signal, which is proportional to the modulus of the truncated Gauss sum $\mathcal{A}_{N}^{(M)}$ introduced in Eq. (2.41).

In Figure 6.5 we compare the experimental data (left) for the number $N=1911$ using pulse sequences containing $M=12$ pulses with the numerical simulation of $\left|\mathcal{A}_{N}^{(M)}\right|$ (right). We find an excellent agreement of theory and experiment.

\subsection{Discussion}

Both experiments discussed in sections 6.1 and 6.2 demonstrate the ability of the truncated Gauss sum $\mathcal{A}_{N}^{(M)}$ to factor numbers.

The implementations base on our proposal of section 4.3 where we have introduced an approach to realize a Gauss sum in the excitation probability amplitude of a two-level system. The resonance condition is varied linearly in time and simultaneously driven by a sequence of weak delta-shaped laser pulses. Moreover, we have shown how to express the number and the argument in terms of the laser parameters. We point out that the Gauss sum arises due to the interference of various quantum paths in the time evolution.

In the following we discuss the differences of both NMR experiments. Both realizations base on a mixed state in equilibrium at room temperature. Here 


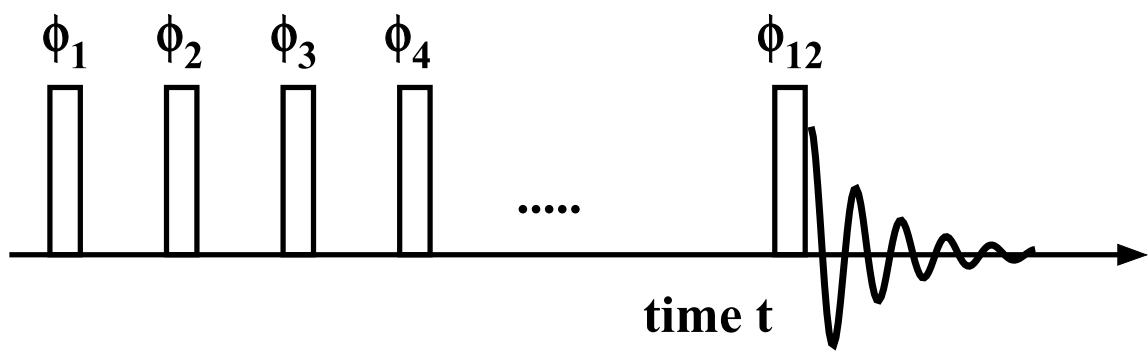

Figure 6.4: Pulse sequence of D. Suter's NMR-experiment.

The RF-pulse sequence covers $M=12$ pulses where the phase of each individual pulse is adjusted to a specific value according to $\phi_{m}$, Eq. (6.22). After the action of the 12-th pulse the accumulated transverse magnetization is measured. The time for each pulse is $1 \mu \mathrm{s}$ and the time between consecutive pulses is $10 \mu \mathrm{s}$. Hence, the duration of a complete pulse sequence for testing a single argument is $132 \mu$ s.
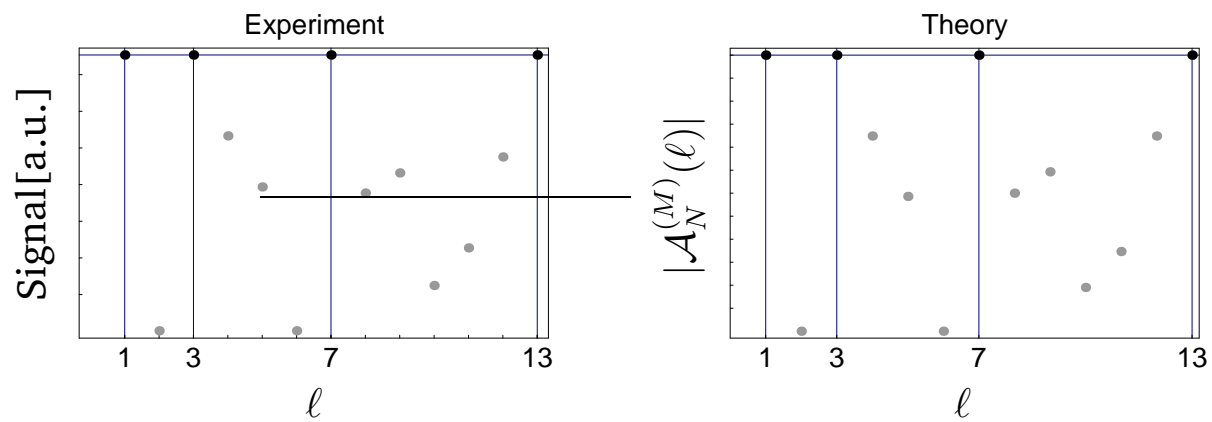

Figure 6.5: Factorization of $N=1911$ by D.Suter's NMR-experiment.

The results of the the NMR-experiment (right) are compared with the numerical simulation (left). We observe an excellent agreement between theoretical predictions and the experimental realization (experimental data courtesy of D. Suter). 
the induced magnetization signal arises as an averaged signal arising from an ensemble of $>10^{19}$ individual non-interacting spins which can be viewed to operate as quantum computers in parallel. In this context the time evolution of the spins cannot be treated perturbatively since the control of this macroscopic spin ensemble requires strong driving fields.

An advantage of ensemble quantum computing manifests itself in the first experiment discussed in section 6.1. Here the measurements hardly perturb the total density matrix of the ensemble as only a small fraction of the spins are projected. Hence, the influence of measurements during the time evolution is not critical.

Next we address the origin of quadratic phase factors and the emerging Gauss sums. In contrast to our original proposal the ratio $N / \ell$ of the number $N$ to be factored and the test integer $\ell$ is precisely controlled by a pulse shaper and does not arise through a pulse-induced time-evolution.

Further differences manifest themselves in the way the Gaussian-type sum emerges. In the first experiment the transverse magnetization $s_{m}$, Eq. (6.16), is acquired after each pulse. The complete signal $\left|\overline{\mathcal{C}}_{N}^{(M)}\right|$, Eq. (6.21), is obtained by averaging the complete set of all $M$ signals $s_{m}$ in a second step on a classical computer.

In contrast, in the second experiment the Gauss sum $\mathcal{A}_{N}^{(M)}$ is directly accessible by a single measurement of the total accumulated magnetization after the last pulse of the sequence. Here the summation is implemented in a physical way as it is automatically performed by the ensemble of spins. 


\section{Conclusions and Outlook}

Conventional approaches to solve the problem of factorizing large numbers base on the implementation of more and more involved sieve methods on a network of parallel classical computers. In contrast, in the present thesis we pursue an alternative route by approaching the factorization problem with a combination of quantum physics and aspects of number theory.

The key to our approach is based on Gauss sums which are central to number theory. For the purpose of factorization we take advantage of the cyclic properties of Gauss sums. The main idea consists in regarding a Gauss sum to arise from the superposition of quantum paths which are weighted by quadratic phase factors. Thus our schemes solely base on interference. Unlike Shor's quantum factoring algorithm our schemes do not feature a speedup since the unique quantum resource of entanglement is not incorporated yet. Nevertheless, this alternative approach might inspire novel evolutions which emerge from combining concepts of quantum physics and number theory.

In the present thesis we have developed three methods for gaining information on the divisors of $N$ from different types of Gauss sums. One method is based on the analysis of a continuously acquired Gauss sum, whereas for the remaining two methods a discrete sampling of the Gauss sum at discrete arguments suffices. The continuous scheme features an intriguing scaling property which allows us to factor also other numbers on the basis of a single experimental realization for $N$.

Moreover, we have presented three quantum optical realizations which we discuss in the context of experimental requirements and limitations. In two of our proposed realizations chirped laser pulses induce one- or two-photon transitions in specific ladder systems which involve a manifold of equidistant states. The resulting excitation probability amplitude arises due to quantum interference of various excitation paths. Due to the chirped pulse each individual quantum path is weighted by a quadratic phase such that the resulting 
total excitation probability amplitude is of the form of the desired Gauss sum. In both cases the Gauss sums are directly accessible via fluorescence measurements from the excited state.

Whereas in the first scheme the quadratic phases arise due to the chirp of the driving field, our last system does not rely on chirped laser pulses. Here the Stark shift causes a linear variation of the resonance condition of a one-photon transition. Simultaneously, a sequence of short pulses drives this transition. Again the resulting excitation probability amplitude involves a Gauss sum. Here the quadratic phase factors emerge due to a linear shift of the excited state energy.

The ability to factor numbers is demonstrated by numerical simulations for each of the proposed realizations.

We also present the key ideas of a recent factorization experiment which is based on the last of our proposed systems. By means of nuclear magnetic resonance Prof. Michael Mehring (University of Stuttgart) has demonstrated the factorization of a six-digit number using sequences of RF-pulses.

The central idea of the present thesis consists of solving a problem of number theory by means of quantum physics. In the following we briefly address extensions of this concept and also provide interesting perspectives.

In this work we have modeled a function from number theory, the Gauss sum, to arise in the dynamics of laser-driven quantum systems. It is interesting that this concept can be transferred to other prominent functions like the Riemann Zeta function $\zeta(s)$. For specific arguments $s, \zeta(s)$ is viewed to arise from the dynamics of a wavepacket in a potential with logarithmic energy spectrum [Dah06, Mai07].

A quantum carpet [Ber01] is the graphical representation of the time evolution of an originally well-localized wavepacket in a one-dimensional box in space and time. Several dominant ridges and valleys with maximal or minimal probability spread over the whole carpet. The slope of these structures is determined by rational fractions of a characteristic time scale. Together with the fact that the underlying energy spectrum is also quadratic we believe that quantum carpets also have the potential to factor numbers.

Moreover, we have found indications that sums over non-linear phases are advantageous compared to sums over linear phase factors [Š07]. With this fact in mind it would be interesting to study a modification of Shor's algorithm which involves as a key step the quantum Fourier transform (QFT). Would a similar transform based on non-linear phase factors be advantageous compared to the standard QFT which involves linear phase factors?

However, the most obvious and at the same time challenging task consists in improving the present factorization scheme by utilizing the unique quantum resource of entanglement. 


\section{APPENDIX A}

\section{Evaluation of Gauss sums}

In this Appendix we consider the generalized Gauss sum

$$
G(a, b) \equiv \sum_{m=0}^{b-1} \exp \left(2 \pi i m^{2} \frac{a}{b}\right)
$$

where $a$ and $b$ do not share any common factor and $b$ has to be an integer.

Many techniques [Lan70, Dav80, Mai07] exist to evaluate the series $G(a, b)$ analytically. Here we summarize the main results which are relevant in this work.

We have

$$
G(a, b)=\left(\frac{a}{b}\right) G(1, b)
$$

where we make use of the Legendre symbol

$$
\left(\frac{a}{b}\right) \equiv\left\{\begin{array}{cl}
+1 & \text { if there is an } x \text { with } b \text { being a divisor of }\left(a-x^{2}\right) \\
-1 & \text { if there is no such } x \\
0 & \text { if } b \text { is a divisor of } a
\end{array}\right.
$$

together with the elementary Gauss sum

$$
G(1, b)=\left\{\begin{array}{cll}
(1+i) \sqrt{b} & \text { for } & b \in \mathcal{M}_{0} \\
\sqrt{b} & \text { for } & b \in \mathcal{M}_{1} \\
0 & \text { for } & b \in \mathcal{M}_{2} \\
i \sqrt{b} & \text { for } & b \in \mathcal{M}_{3}
\end{array}\right.
$$

Here we use the classification of all integers into sets $\mathcal{M}_{k}$ (with $k=0,1, . ., 3$ ), which was defined in Eq. (2.28). 
Another useful property arises from the periodicity intrinsic to the standard Gauss sum $G(a, b)$. If $a$ and $b$ share a common factor $p$ we use the periodicity of the exponentials and split this Gauss sum into $p$ copies of $G(a / p, b / p)$ which are characterized by the period $b / p$ :

$$
G(a, b)=p G\left(\frac{a}{p}, \frac{b}{p}\right)
$$




\section{APPENDIX B}

\section{Gauss sum for broad weight factor distributions}

Here we show that if we impose certain constraints on the weight factor distribution $w_{m}$, the Gauss sum $\mathcal{S}_{N}(\ell)$, Eq. (2.18), reduces to the standard Gauss sum $G(\ell, N)$, which is discussed in more detail in Appendix A.

We derive constraints on the weight factor distribution $w_{m}$. Throughout this Appendix we consider an arbitrary, normalized but slowly varying and broad distribution of weight factors $w_{m}$.

We pursue in two steps: We first cast the sum into a different form and then apply the Fourier theorem. Since the sum

$$
\mathcal{S}_{N}(\ell) \sim \sum_{m=-\infty}^{\infty} w_{m} \exp \left[2 \pi i m^{2} \frac{\ell}{N}\right]
$$

with a weight factor distribution $w_{m}$, is periodic in $n$ with period $N$ we make use of the decomposition

$$
\sum_{m=-\infty}^{\infty} a_{m}=\sum_{p=0}^{r-1} \sum_{n=-\infty}^{\infty} a_{p+n r}
$$

which yields

$$
\mathcal{S}_{N}(\ell)=\sum_{m=0}^{N-1} \exp \left[2 \pi i m^{2} \frac{\ell}{N}\right] \sum_{n=-\infty}^{\infty} w_{m+n N} .
$$

In order to evaluate the second sum we recall the Poisson summation formula

$$
\sum_{n=-\infty}^{\infty} f_{n}=\sum_{\nu=-\infty}^{\infty} \int_{-\infty}^{\infty} d n f(n) e^{2 \pi i \nu n}
$$


with $f(n)$ representing the continuous extension of $f_{n}$ which satisfies $f(n) \equiv f_{n}$ for integer values of $n$.

As a consequence we arrive at

$$
\mathcal{S}_{N}(\ell)=\frac{1}{N} \sum_{\nu=-\infty}^{\infty} \sum_{m=0}^{N-1} \exp \left[2 \pi i\left(m^{2} \frac{\ell}{N}+\frac{m \nu}{N}\right)\right] \tilde{w}\left(\frac{\nu}{N}\right)
$$

where we have defined the Fourier transform

$$
\tilde{w}\left(\frac{\nu}{N}\right) \equiv \int_{-\infty}^{\infty} d x w(x) \exp \left[-2 \pi i \frac{\nu}{N} x\right]
$$

of the weight factors with the continuous extension $w(x)$.

According to the Fourier theorem the product of the widths $\Delta m$ and $\Delta \nu$ of a function $w$ and its associated Fourier transform $\tilde{w}$ is constant.

Since $w_{m}$ is characterized by a large width $1 \ll \Delta m$, the width of the associated Fourier transform $\Delta \nu$ is very small. As a consequence the distribution in $\nu$ is very narrow and the sum over $\nu$ includes essentially only the term $\nu=0$ and we arrive at

$$
\mathcal{S}_{N}(\ell)=\sum_{m=0}^{N-1} \exp \left[2 \pi i m^{2} \frac{\ell}{N}\right],
$$

where we directly identify the result with the standard Gauss sum $G(\ell, N)$. This reduction of the sum to the $\nu=0$-term comes out most clearly for the case of a Gaussian weight factor distribution. 


\section{APPENDIX C}

\section{Pocket factorizer}

Inspired by an idea by Professor Stig Stenholm we found a geometrical visualization of the rescaling property intrinsic to the Gauss sum $\mathcal{S}_{N}$, Eq. (2.1). With this "pocket factorizer" we can factor many numbers $\widetilde{N}$ basing on a single continuously acquired signal $\left|\mathcal{S}_{N}\right|^{2}$ associated with the number $N$. Here $\widetilde{N}$ can be either larger or smaller than $N$. In Figure C. 1 we illustrate the working principle of the pocket factorizer with an example where we reveal the factors of $\widetilde{N}=95$ starting from the original signal $\left|\mathcal{S}_{N}\right|^{2}$ associated with $N=51$. We indicate candidate prime factors by horizontal lines. On the right we depict numbers $\widetilde{N}<N$ and on the bottom numbers $\widetilde{N}>N$. Thus the ratio of numbers $\widetilde{N} / N$ determines the slope of the tilted read-out line.

In order to find the adequate scale for the number $\widetilde{N}$ we adapt the slope of the tilted line accordingly. For testing whether the prime argument $\tilde{\ell}$ is a factor of $\widetilde{N}$ we follow the horizontal line to the crossing with the tilted line. The abscissa $\tilde{\ell}$ of this intersection point yields the region of the signal $\left|\mathcal{S}_{N}\right|^{2}$ which has to be assessed. For this purpose we provide magnified insets of the signal at the position of the prime factors of $N$ and $\widetilde{N}$.

By using this pocket factorizer it is possible to obtain the prime factor resolutions of numbers $\widetilde{N}$ out of the interval from fifteen to about one hundred. 


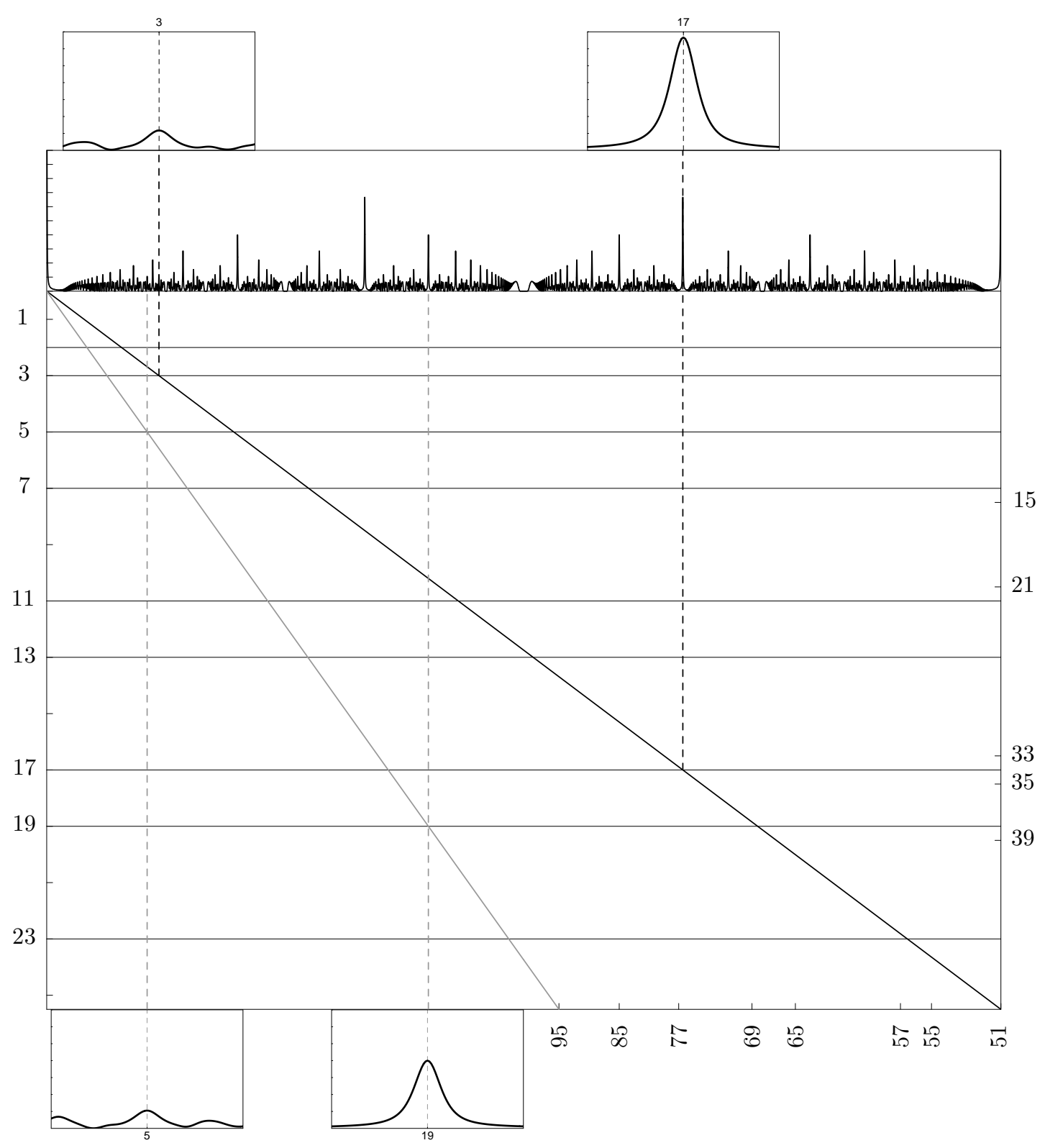

Figure C.1: Graphical solution to the scaling property of Gauss sums.

The pocket factorizer reveals the prime factors of $\widetilde{N}=95$ based on the signal $\left|\mathcal{S}_{N}\right|^{2}$ for $N=51$. Candidate prime factors are depicted by horizontal lines. The numbers on the right and in the bottom denote other numbers $\widetilde{N}$ to be factorized. On the right we depict numbers $\widetilde{N}<N$ and on the bottom numbers $\widetilde{N}>N$. In order to factorize the number $\widetilde{N}$ we have to draw a read-out line through the origin with slope given by the ratio $\widetilde{N} / N$. In order to check whether the prime argument $\tilde{\ell}$ is a factor of $\widetilde{N}$ we follow the horizontal line until the crossing with the tilted line of slope $\widetilde{N} / N$. Each intersection of this line with one of the horizontal lines determines the position where the shape of the signal $\left|\mathcal{S}_{51}\right|^{2}$ has to be analyzed. The insets show the magnified signal at the position of the prime factors of $N$ and $\widetilde{N}$. 


\section{APPENDIX D}

\section{Another type of Gauss sum}

In this Appendix we analytically evaluate the absolute value of the normalized and complete Gauss sum

$$
\mathcal{A}_{N}^{(\ell-1)}(\ell) \equiv \frac{1}{\ell} \sum_{m=0}^{\ell-1} \exp \left[2 \pi i m^{2} \frac{N}{\ell}\right]
$$

where summation range covers the complete period $m=0 \ldots \ell-1$.

The Gauss reciprocity relation [Han80, Mat03] establishes the link to the standard Gauss sum

$$
\mathcal{A}_{N}^{(\ell-1)}(\ell) \equiv \frac{e^{i \frac{\pi}{4}}}{2} \frac{1}{\sqrt{2 \ell N}} G(\ell, 4 N)
$$

of period $4 N$. This identity allows us to fall back on the results of Appendix A for evaluating the signal value as the absolute value of $\mathcal{A}_{N}^{(\ell-1)}(\ell)$.

For each type of integer number $\ell$ we determine the value of the signal $\left|\mathcal{A}_{N}^{(\ell-1)}\right|$. For this purpose we assume that the number $N=p q$ to be factorized is odd and therefore its constituents $p$ and $q$ are also odd.

For each value of $\ell$ we have to check carefully, which of the following cases apply. For this purpose we make use of the fact that every integer number belongs to one of the four sets $\mathcal{M}_{k}$ of numbers, Eq. (2.28).

\section{$\ell$ equals to a factor $p$ of $N$}

Using $N=p q$ we find

$$
G(p, 4 N)=p G(1,4 q)
$$


With $4 q \in \mathcal{M}_{0}$ we arrive at

$$
|G(p, 4 N)|=p|G(1,4 q)|=2 p \sqrt{2 q} .
$$

Thus the absolute value of the complete Gauss sum is given by

$$
\left|\mathcal{A}_{N}^{(\ell-1)}(\ell)\right|=\frac{1}{2} \frac{1}{\sqrt{2 p N}}|G(p, 4 N)|=1 .
$$

$\ell \in \mathcal{M}_{2}$

Here we express the argument as $\ell=4 j+2$. Thus we find

$$
G(4 j+2,4 N)=2 G(j+1,2 N) .
$$

For any odd number $N$ it can be shown that $2 N$ always belongs to the set $\mathcal{M}_{2}$. Hence, for arguments $\ell \in \mathcal{M}_{2}$ both the standard Gauss sum

$$
G(j+1,2 N)=0
$$

and thus also the complete Gauss sum

$$
\mathcal{A}_{N}^{(\ell-1)}(\ell)=0
$$

vanishes.

\section{$\ell$ is not a factor of $N$}

Here we have $4 N \in \mathcal{M}_{0}$ and we arrive at

$$
|G(\ell, 4 N)|=2 \sqrt{2 N} .
$$

Thus we find for the absolute value of the complete Gauss sum

$$
\left|\mathcal{A}_{N}^{(\ell-1)}(\ell)\right|=\frac{1}{2} \frac{1}{\sqrt{2 \ell N}}|G(\ell, 4 N)|=\frac{1}{\sqrt{\ell}} .
$$

\section{$\ell \in \mathcal{M}_{0}$ and $4 N$ share 4 as the only common factor}

Here we have $\ell=4 j$, where $j$ and $N$ are coprime. Thus we find

$$
G(4 j, 4 N)=4 G(j, N)
$$

and consequently

$$
|G(4 j, 4 N)|=4 \sqrt{N}
$$

Hence the complete Gauss sum takes on the value

$$
\left|\mathcal{A}_{N}^{(\ell-1)}(\ell)\right|=\frac{1}{2} \frac{1}{\sqrt{2 \ell N}}|G(4 j, 4 N)|=\sqrt{\frac{2}{\ell}} .
$$




\section{$\ell$ and $N$ share a common factor $p$}

Here we have $\ell=j p$ and $N=q p$ with the constraint that $j$ is not a multiple of 4. Using the periodicity of the Gauss sum we find

$$
G(j p, 4 q p)=p G(j, 4 q)
$$

and hence

$$
|G(j p, 4 q p)|=p \sqrt{2} \sqrt{4 q}=2 p \sqrt{2 q} .
$$

Thus we find for the absolute value of the Gauss sum

$$
\left|\mathcal{A}_{N}^{(\ell-1)}(\ell)\right|=\frac{1}{2} \frac{1}{\sqrt{2 \ell N}}|G(j p, 4 q p)|=\frac{1}{\sqrt{j}} .
$$

\section{$\ell \in \mathcal{M}_{0}$ and $4 N$ share a common factor $4 p$}

Here we have $\ell=4 j p \in \mathcal{M}_{0}$ and $N=q p$ and consequently

$$
G(4 j p, 4 q p)=4 p G(j, q) \text {. }
$$

Using the fact that $q$ is odd we find

$$
|G(4 j p, 4 q p)|=4 p \sqrt{q}
$$

and hence the modulus of the complete Gauss sum is given by

$$
\left|\mathcal{A}_{N}^{(\ell-1)}(\ell)\right|=\frac{1}{2} \frac{1}{\sqrt{2 j p N}}|G(4 j p, 4 q p)|=\frac{1}{\sqrt{2 j}} .
$$




\section{APPENDIX E}

\section{Asymptotic expansion of the complementary error function}

In this Appendix we derive the asymptotic behavior for the complementary error function

$$
\operatorname{erfc}\left(\zeta_{m}\right)=\frac{2}{\sqrt{\pi}} \int_{\zeta_{m}}^{\infty} d u e^{-u^{2}}
$$

where the integration limits $\zeta_{m}=i \delta_{m} /\left(2 f_{0}\right)$ and " $\infty$ " are in general complexvalued. We restrict ourselves to cases where the offset $\delta_{m}$ of the $m$-th intermediate state $|m\rangle$ in the equidistant manifold is positive, that is $\delta_{m}>0$.

We note that the integrand $\exp \left(-u^{2}\right)$ is analytic in the whole complex plane. For angles smaller than $\pm \pi / 4$ the integrand decreases exponentially. Both decaying domains are connected via a saddle at the origin. Outside this saddle the integrand grows exponentially as we move outward along the positive and negative imaginary axis. In the surrounding sector, that is for angles from $\pi / 4$ to $3 \pi / 4$ (or $-\pi / 4$ to $-3 \pi / 4$ ) we also deal with an exponentially growing integrand.

For the following it is convenient to express the lower integration limit

$$
\zeta_{m} \equiv\left|\zeta_{m}\right| e^{i \beta}
$$

in polar-representation where we introduce the real-valued amplitude

$$
\left|\zeta_{m}\right| \equiv \frac{\delta_{m}}{\Delta \omega}\left(1+a^{2}\right)^{1 / 4}
$$

and the phase

$$
\beta=\frac{1}{2}(\pi-\arctan a) .
$$


Moreover, the present derivation assumes finite values for the offset $\delta_{m}$ and the bandwidth $\Delta \omega$ of the chirped pulse.

The sign of the dimensionless chirp $a$ critically determines the position of the lower integration bound

$$
\zeta_{m}=\left|\zeta_{m}\right| \begin{cases}\exp \left[i\left(\frac{\pi}{4}+\frac{\epsilon}{2}\right)\right] & \text { for } a>0 \\ \exp \left[i\left(\frac{3 \pi}{4}-\frac{\epsilon}{2}\right)\right] & \text { for } a<0\end{cases}
$$

in the complex plane. Here we consider the case of $|a| \rightarrow \infty$ together with $0<\epsilon \ll 1$.

For large positive values of $a>0 \zeta_{m}$ is positioned slightly above the diagonal in the first quadrant. For negative values of $a<0 \zeta_{m}$ is in the second quadrant slightly above the orthogonal diagonal. We remark that for both values of the sign of $a$ the starting point $\zeta_{m}$ is located in the domain where the integrand increases exponentially.

In this context we analyze how the integration path approaches infinity. The behavior of

$$
\frac{\Delta \omega}{2} f_{0}=\frac{\Delta \omega}{2}(1-i a)^{-1 / 2}=\frac{\delta_{m}}{2\left|\zeta_{m}\right|} \exp \left[\frac{i}{2} \arctan a\right]
$$

critically determines whether the integral converges. For larger positive or negative values of $0 \ll|a|$ we find the asymptotic behavior

$$
\frac{\Delta \omega}{2} f_{0}=\frac{\delta_{m}}{2\left|\zeta_{m}\right|}\left\{\begin{array}{ll}
\exp \left[i\left(\frac{\pi}{4}-\frac{\epsilon}{2}\right)\right] & \text { for } a>0 \\
\exp \left[i\left(-\frac{\pi}{4}+\frac{\epsilon}{2}\right)\right] & \text { for } a<0
\end{array}\right. \text {. }
$$

Thus for $a>0$ the lower integration limit $\zeta_{m}$ is located in the first quadrant and the direction of approaching $+\infty$ is less steep than the first diagonal. Thus the path of integration proceeds almost completely in a domain where the integrand shows a decaying behavior.

For the other case of negative values of $a$ the path of integration starts in the second quadrant and proceeds with a slope slightly steeper than the second diagonal. After crossing the saddle oriented in the direction of the imaginary axis the integration path proceeds to $\infty$ in the decaying domain in the direction of the second diagonal.

In Figure E.1 we illustrate the different location of the lower integration bound $\zeta_{m}$ for $a>0$ and $a<0$ in the complex plane. Moreover, we indicate the direction of both integration paths $\mathcal{C}_{0}$ and $\mathcal{C}_{1}$ in the complex plane. 


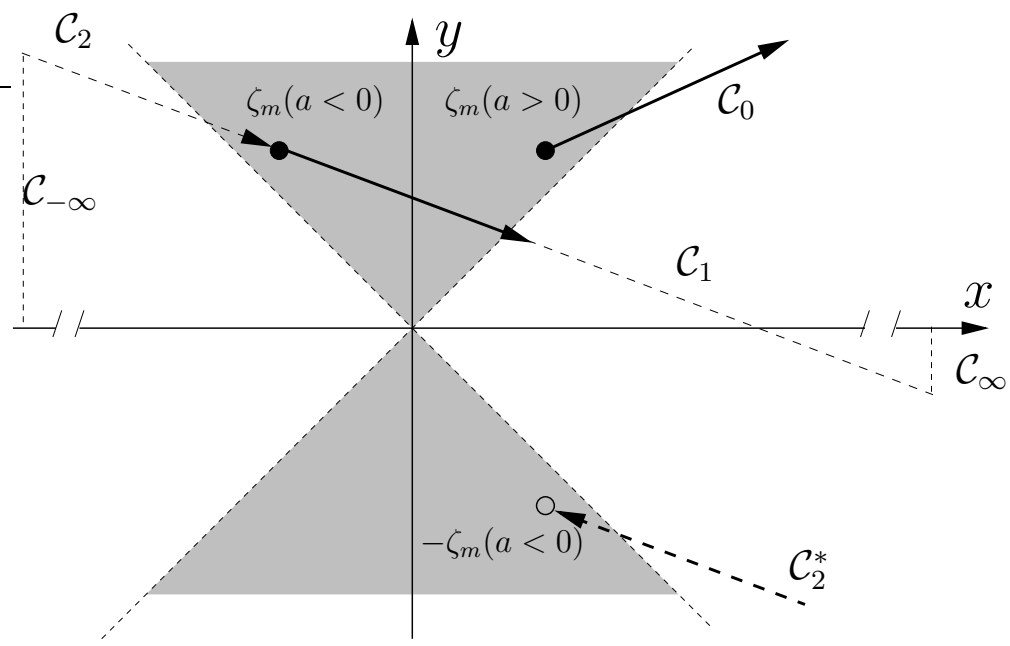

Figure E.1: Integration paths $\mathcal{C}_{0}$ and $\mathcal{C}_{1}$ in the complex plane.

We depict the domains in complex plane where the integrand increases exponentially by the shaded area. For $a>0$ the lower integration limit $\zeta_{m}$ is located in the first quadrant and the direction of approaching $+\infty$ is less steep than the first diagonal. Thus the path of integration $\mathcal{C}_{0}$ proceeds almost completely in the domain of complex plane where the integrand shows a decaying behavior. For the other case of negative values of a the path of integration starts in the second quadrant and proceeds with a slope slightly steeper than the second diagonal. After crossing the saddle oriented along the imaginary axis the integration path $\mathcal{C}_{1}$ evolves along the second diagonal in the decaying region. 


\section{E.1 Integration path for $a>0$ : decaying regime}

As first case we discuss here the integral

$$
\operatorname{erfc}\left(\zeta_{m}\right)=\frac{2}{\sqrt{\pi}} \int_{\mathcal{C}_{0}} d u e^{-u^{2}}
$$

for positive values of $a$. We have already remarked that in this case the integration path starts in the first quadrant and proceeds almost completely in the part of the complex plane where the integrand shows a decaying behavior.

An integration by parts yields

$$
\operatorname{erfc}\left(\zeta_{m}\right)=\frac{1}{\sqrt{\pi}}\left(-\left.\frac{e^{-u^{2}}}{u}\right|_{\zeta_{m}} ^{\infty}+\int_{\zeta_{m}}^{\infty} d u \frac{e^{-u^{2}}}{u}\right)
$$

and consequently

$$
\operatorname{erfc}\left(\zeta_{m}\right)=\frac{e^{-\zeta_{m}^{2}}}{\sqrt{\pi} \zeta_{m}}+\frac{1}{\sqrt{\pi}} \int_{\zeta_{m}}^{\infty} d u \frac{e^{-u^{2}}}{u}
$$

We note that the remaining integral can be repeatedly integrated by parts thus yielding a series of higher order correction terms. When we keep only the first term we arrive at the approximation

$$
\operatorname{erfc}\left(\zeta_{m}\right) \approx \frac{1}{\sqrt{\pi}} \frac{e^{-\zeta_{m}^{2}}}{\zeta_{m}}
$$

valid for positive values of $a$.

\section{E.2 Integration path for $a<0$ : oscillatory regime}

The second case for $a<0$ is a bit more involved. Here the integration

$$
\operatorname{erfc}\left(\zeta_{m}\right)=\frac{2}{\sqrt{\pi}} \int_{\mathcal{C}_{1}} d u e^{-u^{2}}
$$

starts in the second quadrant and the path $\mathcal{C}_{1}$ of approaching $\infty$ is not solely in a domain where the integrand shows a decaying behavior.

For this case we consider a closed integration path as depicted by the dashed line in Figure E.1. From $\zeta_{m}$ (in the second quadrant) we follow the path $\mathcal{C}_{1}$ to $\infty$, go up to the real axis along $\mathcal{C}_{\infty}$, follow the real axis to $-\infty$, go up vertically 
along the path $\mathcal{C}_{-\infty}$ and finally follow $\mathcal{C}_{2}$ to the starting point $\zeta_{m}$. Due to the exponential decay of the integrand at $\pm \infty$ both contributions of the "vertical" integration paths $\mathcal{C}_{\infty}$ and $\mathcal{C}_{-\infty}$ vanish.

When we apply the Cauchy integral relation

$$
\int_{\mathcal{C}_{1}} d u e^{-u^{2}}+\int_{\infty}^{-\infty} d x e^{-x^{2}}+\int_{\mathcal{C}_{2}} d u e^{-u^{2}}=0
$$

we express the integral of interest

$$
\int_{\mathcal{C}_{1}} d u e^{-u^{2}}=\sqrt{\pi}-\int_{\mathcal{C}_{2}} d u e^{-u^{2}}
$$

in terms of an integration along the real axis and the integration which approaches $\zeta_{m}$ from $-\infty$ along the path $\mathcal{C}_{2}$.

By a substitution we "mirror" the latter integral to an integration path $\mathcal{C}_{2}^{*}$ which proceeds in the decaying domain of the forth quadrant, thus connecting $+\infty$ and

$$
-\zeta_{m}(a<0)=\left|\zeta_{m}\right| \exp \left[-i\left(\frac{\pi}{4}+\frac{\epsilon}{2}\right)\right] .
$$

As in the previous section for $a>0$ we evaluate this integration along $\mathcal{C}_{2}^{*}$ by partial integration.

Hence, we obtain the approximation

$$
\operatorname{erfc}\left(\zeta_{m}\right) \approx 2+\frac{1}{\sqrt{\pi}} \frac{e^{-\zeta_{m}^{2}}}{\zeta_{m}}
$$

for negative values of $a$.

\section{E.3 Asymptotic expressions}

We combine both asymptotic expressions Eq. (E.11) and Eq. (E.16):

$$
\operatorname{erfc}\left(\zeta_{m}\right) \approx\left\{\begin{aligned}
2+\frac{1}{\sqrt{\pi}} \frac{e^{-\zeta_{m}^{2}}}{\zeta_{m}} & \text { for } a<0 \\
\frac{1}{\sqrt{\pi}} \frac{e^{-\zeta_{m}^{2}}}{\zeta_{m}} & \text { for } a>0
\end{aligned}\right.
$$

where we recall that this result holds true for $\delta_{m}>0$ and that the argument $\zeta_{m}$ depends on $a$ via Eq. (E.3) and Eq. (E.4). 
In the following we analyze the asymptotic behavior for $|a| \rightarrow \infty$. For this purpose it is useful to rewrite Eq. (E.17)

$$
\operatorname{erfc}\left(\zeta_{m}\right) \approx\left\{\begin{aligned}
2+\frac{e^{-\left|\zeta_{m}\right|^{2} \cos (2 \beta)}}{\sqrt{\pi}\left|\zeta_{m}\right|} \exp \left[-i\left(\left|\zeta_{m}\right|^{2} \sin (2 \beta)+\beta\right)\right] & \text { for } a<0 \\
\frac{e^{-\left|\zeta_{m}\right|^{2} \cos (2 \beta)}}{\sqrt{\pi}\left|\zeta_{m}\right|} \exp \left[-i\left(\left|\zeta_{m}\right|^{2} \sin (2 \beta)+\beta\right)\right] & \text { for } a>0
\end{aligned}\right.
$$

in terms of the absolute value $\left|\zeta_{m}\right|$ and phase $\beta$ of $\zeta_{m}$. In the limit of large positive (or negative values) of $a$ the phase $\beta$ approaches $\pi / 4$ (or $3 \pi / 4$ ) while $\left|\zeta_{m}\right|$ increases to infinity independent on the sign of $a$. With these facts Eq. (E.18) simplifies considerably and we arrive at

$$
\operatorname{erfc}\left(\zeta_{m}\right) \approx \begin{cases}2 & \text { for } a \rightarrow-\infty \\ 0 & \text { for } a \rightarrow \infty\end{cases}
$$

In Figure E. 2 we compare the absolute value and the phase of our approximation with the exact result. For $1<|a|$ we find an excellent agreement of the approximation, Eq. (E.18). For $a<0$ the phase reveals slight oscillations around zero while the absolute value oscillates around 2 with a decreasing amplitude as $a$ tends to $-\infty$. In contrast, for positive values of $a$ the absolute value decays to 0 .

For $\delta_{m}>0$ and for large values of $a$ we arrive at the approximation

$$
\operatorname{erfc}\left(\zeta_{m}\right) \approx 2 \Theta\left(-\delta_{m} a\right)
$$

of the complementary error function by means of the Heaviside step function. 

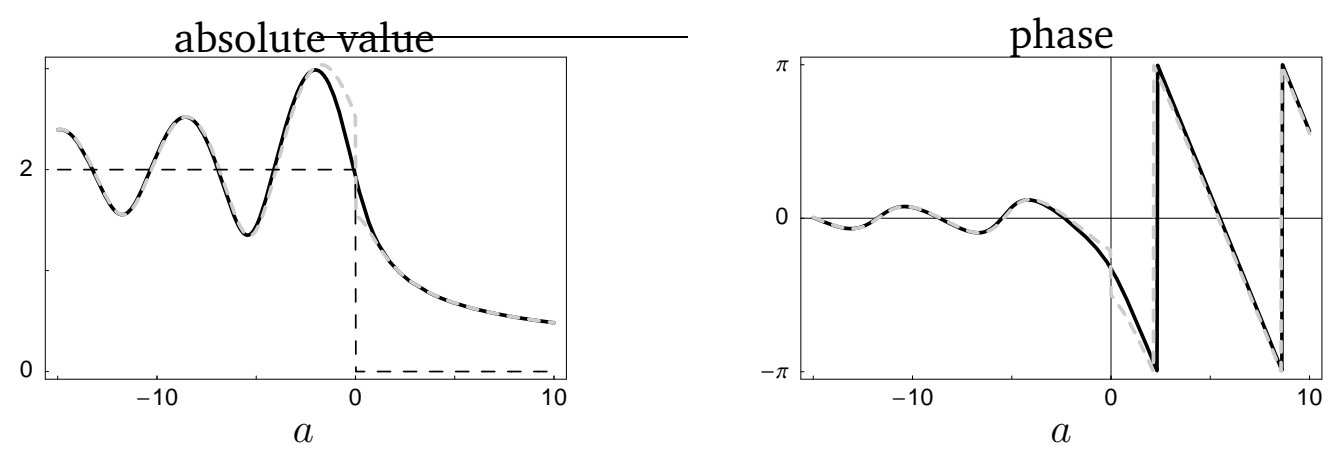

Figure E.2: Comparison of the complementary error function $\operatorname{erfc}\left(\zeta_{m}\right)$ (black) and the approximation, Eq. (E.17) (dashed gray line).

On the left we depict the absolute value and on the right the phase. Outside $a=0$ we find an excellent agreement of both curves. For $a<0$ the phase reveals slight oscillations around zero while the absolute value oscillates around 2 with a decreasing amplitude as a tends to $-\infty$. In contrast, for positive values of a the absolute value decays to 0 . In addition, we depict the approximation by the dashed line in the left plot by means of the Heaviside step function, Eq. (E.20), which describes the behavior in the limit of $|a| \rightarrow \infty$. Here we chose the parameters such that $\delta_{m} / \Delta \omega=1$. 


\section{Bibliography}

[Abr72] M. ABRAmowitz and I. A. STEgUn, Handbook of mathematical functions (Dover Publications, New York, 1972).

[Agr04] M. Agrawal, N. Kayal, and N. SaXena, "PRIMES is in P", Annals of Mathematics 160, pp. 781 (2004).

[Ash96] R. C. Ashoori, "Electrons in artificial atoms", Nature 379, p. 413 (1996).

[Ave89] I. S. Averbukh and N. F. Perelman, "Fractional revivals: Universality in the long-term evolution of quantum wave packets beyond the correspondence principle dynamics", Phys. Lett. A 139, p. 449 (1989).

[Bal94] P. BAlling, D. J. MAAS, and L. D. NOORDAM, "Interference and climbing a quantum ladder system with frequency-chirped pulses", Phys. Rev. A 50, p. 4276 (1994).

[Bei64] A. BEILER, Recreations in the Theory of Numbers (Dover Publications, 1964).

[Ber88a] M. V. BERRY, "Random renormalization in the semiclassical longtime limit of a precessing spin", Physica D 33, pp. 26 (1988).

[Ber88b] M. V. BERRY and J. GOLDBERG, "Renormalization of curlicues", Nonlinearity 1, pp. 1 (1988).

[Ber98] K. Bergmann, H. Theuer, and B. Shore, "Coherent population transfer among quantum states of atoms and molecules", Rev. Mod. Phys. 70, p. 1003 (1998). 
[Ber01] M. Berry, I. MARZOLI, and W. P. SCHLEICH, "Quantum carpets, carpets of light", Physics World 14, p. 39 (2001).

[Bou00] D. Bouwmeester, A. Ekert, and A. Zeilinger, editors, The Physics of Quantum Information (Springer Berlin, 2000).

[Bro92] B. Broers, H. B. VAN Linden van Den Heuvell, and L. D. NoorDAM, "Efficient population transfer in a three-level ladder system by frequency-swept ultrashort laser pulses", Phys. Rev. Lett 69, p. 2062 (1992).

[Car54] H. Y. CARR and E. M. PuRCELl, "Effects of Diffusion on Free Precession in Nuclear Magnetic Resonance Experiments", Phys. Rev. 94, pp. 630 (1954).

[Cha03] B. Chatel, J. Degert, S. Stock, and B. Girard, "Competition between sequential and direct paths in a two-photon transition", Phys. Rev. A 68, p. 041402(R) (2003).

[Che90] S. Chelkowski, A. D. Bandrauk, and P. B. Corkum, "Efficient molecular dissociation by a chirped ultrashort infrared laser pulse", Phys. Rev. Lett. 65, pp. 2355 (1990).

[Cir95] J. I. CiraC and P. Zoller, "Quantum Computations with Cold Trapped Ions”, Phys. Rev. Lett. 74, pp. 4091 (1995).

[Cla96] J. F. Clauser and J. P. Dowling, "Factoring integers with Young's $N$-slit interferometer", Phys. Rev. A 53, p. 4587 (1996).

[Dah06] J. P. DAhl, R. Mack, H. Moya-Cessa, W. Strunz, R.WAlser, and W. P. SCHLEICH, "Riemann Zeta function from wave packet dynamics", (2006), in preparation for Phys. Rev. A.

[Dav80] H. DAVEnPoRT, Multiplicative Number Theory (Springer, New York, 1980).

[Deg02] J. Degert, W. Wohlleben, B. Chatel, M. Motzkus, and B. GiRARD, "Realization of a Time-Domain Fresnel Lens with Coherent Control", Phys. Rev. Lett. 89, pp. 203003 (2002).

[Der03] J. Derbyshire, Prime Obsession: Bernhard Riemann and the greatest unsolved problem in mathematics (Penguin group, New York, 2003).

[Die02] R. B. Diener, B. Wu, M. G. RAIZEN, and Q. NiU, "Quantum Tweezer for Atoms", Phys. Rev. Lett. 89, p. 070401 (2002). 
[DiV00] D. P. DiVincenzo, "The Physical Implementation of Quantum Computation", Fortschr. Phys. 48, pp. 771 (2000).

[Eke96] A. EKERT and R. JozSA, "Quantum computation and Shor's factoring algorithm”, Rev. Mod. Phys. 68, pp. 733 (1996).

[Ern87] R. R. Ernst, G. Bodenhausen, and A. Wokaun, Principles of Nuclear Magnetic Resonance in One and Two Dimensions (Clarendon Press, Oxford, 1987).

[EUR06] "Quantum Information Processing and Communication, Strategic report on current status, visions and goals for research in Europe", (2006), URL http://qist.ect.it.

[Fey82] R. P. FEYNMAN, "Simulating physics with computers", Int. J. Theor. Phys. 21, pp. 467 (1982).

[Gal88] T. F. Gallagher, "Rydberg atoms", Rep. Prog. Phys. 51, pp. 143 (1988).

[Ger05] E. GERJUOY, "Shor's factoring algorithm and modern cryptography. An illustration of the capabilities inherent in quantum computers", Am. J. Phys. 73, p. 521 (2005).

[Gre02] M. Greiner, O. Mandel, T. Esslinger, T. W. Hänsch, and I. BLOCH, "Quantum Phase Transition from a Superfluid to a Mott Insulator in a Gas of Ultracold Atoms", Nature 415, pp. 39 (2002).

[Gri97] M. Grifoni and P. HÄNGGI, "Driven Quantum Tunneling", Phys. Rep. 304, p. 229 (1997).

[Gul03] S. Gulde, M. Riebe, G. P. T. Lancaster, C. Becher, J. Eschner, H. HÄFfner, F. Schmidt-Kaler, I. L. Chuang, and R. Blatt, "Implementation of the Deutsch-Jozsa algorithm on an ion-trap quantum computer", Nature 421, pp. 48 (2003).

[Han80] J. H. HANNAY and M. V. BERRY, "Quantization of linear maps on a torus-Fresnel diffraction by a periodic grating", Physica D. 1, pp. 267 (1980).

[Har01] W. G. HARTER, "Quantum-fractal revival structure in $C_{N}$ quadratic spectra: Base- $N$ quantum computer registers", Phys. Rev. A 64, p. 012312 (2001).

[Jak98] D. JAKsCh, C. Bruder, J. I. CiraC, C. W. GARdiner, and P. Zoller, "Cold Bosonic Atoms in Optical Lattices", Phys. Rev. Lett. 81, pp. 3108 (1998). 
[Jan03] E. JANÉ, G. VidAl, W. DÜR, P. Zoller, and J. I. CiraC, "Simulation of quantum dynamics with quantum optical systems", Quantum Information and Computation 3, pp. 15 (2003).

[Kan98] B. E. KANE, "A silicon-based nuclear spin quantum computer", Nature 393, pp. 133 (1998).

[Kip97] R. KipPENHAHn, Verschlüsselte Botschaften, Geheimschriften, Enigma und Chipkarte (Rowohlt Taschenbuch Verlag, Hamburg, 1997).

[Lan70] S. LANG, Algebraic Number Theory (Addison Wesley, New York, 1970).

[Lav03] C. LAVor, L. MANSSUR, and R. Portugal, "Shor's algorithm for Factoring Large Integers", (2003), quant-ph/0303175.

[Leh14] D. N. LEHMER, List of primes numbers from 1 to 10,006,721 (Carnegie Institution Washington, D.C., 1914).

[Lei96a] C. Leichtle, I. S. Averbukh, and W. P. Schleich, "Generic Structure of Multilevel Quantum Beats”, Phys. Rev. Lett. 77, p. 3999 (1996).

[Lei96b] C. Leichtle, I. S. Averbukh, and W. P. Schleich, "Multilevel quantum beats: An analytical approach", Phys. Rev. A 54, pp. 5299 (1996).

[Lei03] D. Leibfried, R. Blatt, C. Monroe, and D. Wineland, "Quantum dynamics of single trapped ions”, Rev. Mod. Phys 75, pp. 281 (2003).

[Llo96] S. LlOYD, "Universal Quantum Simulators", Science 273, pp. 1073 (1996).

[Los98] D. Loss and D. P. DiVInCEnZO, "Quantum computation with quantum dots", Phys. Rev. A 57, p. 120 (1998).

[Maa99] D. J. MAas, C. W. Rella, P. Antoine, E. S. Toma, and L. D. NoorDAM, "Population transfer via adiabatic passage in the rubidium quantum ladder system", Phys. Rev. A 59, p. 1474 (1999).

[Mac02a] H. Mack, M. Bienert, F. Haug, M. Freyberger, and W. P. SchleICH, "Wave Packets Can Factorize Numbers", phys. stat. sol. (b) 233(3), pp. 408 (2002).

[Mac02b] H. Mack, M. Bienert, F. Haug, F. S. Straub, M. Freyberger, and W. P. SCHLEICH, "Wave packet dynamics and factorization of numbers", in Proceedings of the Enrico Fermi Summer School, Course CXLVIII: Experimental Quantum Computation, Eds. P. Mataloni and F. De Martini, Elsevier, Amsterdam pp. 369-383 (2002). 
[Mah07] T. S. Mahesh, N. Rajeendran, X. Peng, and D. Suter, "Factorizing Numbers with the Gauss Sum Technique: NMR Implementations", (2007), quant-ph/0701205v1.

[Mai07] H. MAIER and W. P. SchleICH, Prime Numbers 101: A Primer on Number Theory (Wiley-VCH, New York, 2007), to be published.

[Mak01] Y. MAKHLIN, G. SchÖN, and A. ShNiRMAn, "Quantum-state engineering with Josephson-junction devices", Rev. Mod. Phys. 73, pp. 357 (2001).

[Mat03] S. MATSUTANi and Y. ONISHI, "Wave-Particle Complementary and Reciprocity of Gauss sums on Talbot Effects", Foundations of Physics Letters 16(4), pp. 325 (2003).

[Meh07] M. Mehring, K. Müller, I. Sh. Averbukh, W. Merkel, and W. P. SCHLEICH, "NMR experiment factors numbers with Gauss sums", Phys. Rev. Lett. 98, p. 120502 (2007).

[Mei58] S. Meiboom and D. GiLL, "Effects of Diffusion on Free Precession in Nuclear Magnetic Resonance Experiments", Rev. Sci. Inst. 29, p. 688 (1958).

[Mer06] W. Merkel, O. Crasser, F. Haug, E. Lutz, H. Mack, M. FreyBerger, W. P. Schleich, I. Sh. Averbukh, M. Bienert, B. Girard, H. MAier, and G. G. Paulus, "Chirped pulses, Gauss sums and the factorization of numbers", Int. J. of Mod. Phys. B 20(11-13), pp. 1893 (2006).

[Moh05] B. Mohring, M. Bienert, F. Haug, G. Morigi, W. P. Schleich, and M. G. RAIZEN, "Extracting atoms on demand with lasers", Phys. Rev. A 71, p. 053601 (2005).

[Mon06] A. Monmayrant, B. Chatel, and B. Girard, "Quantum State Measurement Using Coherent Transients", Phys. Rev. Lett. 96, pp. 103002 (2006).

[Nie00] M. A. Nielsen and I. L. ChUANG, Quantum Computation and Quantum Information (Cambridge University Press, 2000).

[Oos98] T. H. Oosterkamp, T. FujisaWA, W. G. VAN DER Wiel, K. ISHibashi, R. V. Hijman, S. Tarucha, and L. P. Kounenhoven, "Microwave spectroscopy of a quantum-dot molecule", Nature 395, p. 873 (1998).

[Ore84] J. Oreg, F. T. Hioe, and J. H. EBERLY, "Adiabatic following in multilevel systems", Phys. Rev. A 29, p. 690 (1984). 
[Par86] J. PARKER and C. R. STROUd, "Coherence and decay of Rydberg wave Packets", Phys. Rev. Lett. 56, pp. 716 (1986).

[Phi98] W. D. PhILlips, "Laser cooling and trapping of neutral atoms", Rev. Mod. Phys. 70, p. 721 (1998).

[Por04] D. PORRAS and J. CirAC, "Effective quantum spin systems with ion traps”, Phys. Rev. Lett. 92, p. 207901 (2004).

[Rab00] H. Rabitz, R. DE Vivie-Riedle, M. Motzkus, and K. Kompa, "Whither the Future of Controlling Quantum Phenomena?", Science 288, pp. 824 (2000).

[Rai01] J. M. RAImond, M. BRUne, and S. HAROCHE, "Manipulating quantum entanglement with atoms and photons in a cavity", Rev. Mod. Phys. 73, pp. 565 (2001).

[Ran05] A. A. Rangelov, N. V. Vitanov, L. P. Yatsenko, B. W. Shore, T. HALFMANN, and K. BERGMANN, "Stark-shift-chirped rapid-adiabaticpassage technique among three states", Phys. Rev. A 72, p. 053403 (2005).

[Rie04] M. Riebe, H. HÄFfner, C. F. Roos, W. HÄnsel, J. Benhelm, G. P. T. Lancaster, T. W. KÖrber, C. Becher, F. Schmidt-Kaler, D. F. V. JAMES, and R. BLATT, "Deterministic quantum teleportation with atoms", Nature 429, pp. 734 (2004).

[Riv78] R. Rivest, A. Shamir, and L. Adleman, "A Method for Obtaining Digital Signatures and Public-Key Cryptosystems", Communications of the ACM 21, pp. 120 (1978).

[Roo04] C. F. Roos, M. Riebe, H. HÄFfner, W. HÄnsel, J. BenhelM, G. P. T. LANCAster, C. Becher, F. Schmidt-Kaler, and R. Blatt, "Control and Measurement of Three-Qubit Entangled States", Science 304, pp. 1478 (2004).

[Sar74] M. SARgent, M. O. Scully, and W. E. LAmb, Laser Physics (AddisonWesley, Reading, 1974).

[Sch01] W. P. SchleICH, Quantum Optics in Phase Space (Wiley VCH, Berlin, 2001).

[Sha00] M. Shapiro and P. Brumer, "Coherent Control", Adv. At. Mol. Opt. Phys. 42, p. 287 (2000).

[Shi65] J. H. SHIRLEY, "Solution of the Schrödinger Equation with a Hamiltonian Periodic in Time", Phys. Rev. 138, pp. B979 (1965). 
[Sho90] B. W. SHORE, Theory of Coherent Atomic Excitation (John Wiley \& Sons, 1990).

[Sho94] P. SHOR, "Algorithms for Quantum Computation: Discrete Logarithms and Factoring", in Proceedings of the 35th Annual Symposium on Foundations of Computer Science, Santa Fe, NM, edited by S. Goldwasser (IEEE Computer Society Press, New York) pp. 124-134 (1994).

[Sho97] P. SHOR, "Polynomial-Time Algorithms for Prime Factorization and Discrete Logarithms on a Quantum Computer", SIAM Journal of Computing 26, pp. 1484 (1997).

[Sin00] S. SingH, Geheime Botschaften. Die Kunst der Verschlüsselung von der Antike bis in die Zeiten des Internet. (Carl Hanser Verlag, München, 2000).

[Ste97] A. STEANE, "The ion trap quantum information processor", Appl. Phys. B 64, pp. 623 (1997).

[Ste05] S. Stenholm and K.-A. Suominen, Quantum Approach to Informatics (John Wiley \& Son, Inc. Hoboken, New Jersey, 2005).

[Tia05] L. Tian, R. BlatT, and P. ZOLLER, "Scalable ion trap quantum computing without moving ions", Eur. Phys. J. D 32, p. 201 (2005).

[USR04] "A Quantum Information Science and Technology Roadmap", (2004), URL http://quist. lanl.gov.

[Van01] L. M. K. Vandersypen, M. Steffen, G. Breyta, C. S. Yannoni, M. H. SHERWOOD, and I. L. CHUANG, "Experimental realization of Shor's quantum factoring algorithm using nuclear magnetic resonance", Nature 414, pp. 883 (2001).

[Van05] L. M. K. VANDERSYPEn and I. L. ChUANG, "NMR techniques for quantum control and computation", Rev. Mod. Phys. 76(4), pp. 1037 (2005), see also references therein.

[Vit01] N. V. Vitanov, T. Halfmann, B. W. Shore, and K. Bergmann, "Laser-induced population transfer by adiabatic passage techniques", Ann. Rev. Phys. Chem. 52, p. 763 (2001).

[Vol01] I. VolOVICH, "Quantum Computing and Shor's algorithm", (2001), quant-ph/0109004.

[ŠO6] M. Štefañák, W. Merkel, W. P. Schleich, D. HaAse, and H. Maier, "Optimal truncation of Gauss sums for integer factorization", (2006), in preparation for N. J. P. 
[Š07] M. ŠTEFAŇÁK, W. MERKEL, and W. P. SCHLEICH, "NMR realization of sums over nonlinear phase factors for integer factorization", (2007), in preparation for J. Mod. Opt.

[Wal02] S. Wallentowitz, I. A. Walmsley, L. J. WaXer, and T. Richter, "Rotationally induced collapse and revivals of molecular vibrational wavepackets: model for environment-induced decoherence", J. Phys. B: At. Mol. Opt. Phys. 35, pp. 1967 (2002).

[War80] W. S. Warren, D. P. Weitekamp, and A. Pines, "High Order Selective Sequences in Multiple Quantum NMR", J. Magn. Reson. 40, pp. 581 (1980).

[Wie99] C. E. Wieman, D. E. Pritchard, and D. J. Wineland, "Atom cooling, trapping, and quantum manipulation", Rev. Mod. Phys. 71, p. 253 (1999).

[Wun06] C. Wunderlich, T. Hannemann, T. Koerber, H. Haeffner, C. Roos, W. HAEnsel, R. Blatt, and F. SchmidT-Kaler, "Robust state preparation of a single trapped ion by adiabatic passage", Journal of Modern Optics (2006), quant-ph/0508159.

[Za198] C. ZALKA, "Simulating Quantum Systems on a Quantum Computer", Phil. Trans. R. Soc. Lond. A 454, pp. 313 (1998).

[Zam01] S. Zamith, J. Degert, S. Stock, B. De Beauvoir, V. Blanchet, M. Aziz Bouchene, and B. GiRARD, "Observation of Coherent Transients in Ultrashort Chirped Excitation of an Undamped Two-Level System", Phys. Rev. Lett. 87, pp. 033001 (2001).

[Zim79] M. L. Zimmerman, M. G. Littman, M. M. Kash, and D. Kleppner, "Stark structure of the Rydberg states of alkali-metal atoms", Phys. Rev. A 20, pp. 2251 (1979). 


\section{Zusammenfassung}

Heute ist das Internet unverzichtbar in der weltweiten Kommunikation und zum Austausch von Daten. Speziell im Bereich e-commerce ist die Sicherheit bei der Übermittlung von Daten zwischen zwei Parteien unerlässlich. Bisher wird die Sicherheit durch moderne Kryptographiesysteme gewährleistet. Als eines der ersten asymmetrischen Verfahren wurde Ende der siebziger Jahre das RSAProtokoll entwickelt. Multiplikation und Faktorisierung bilden ein Funktionenpaar, dessen Implementierung auf klassischen Computern hoch asymmetrisch im Aufwand ist. Während dieser für die Multiplikation von großen Primzahlen unerheblich ist, skaliert der Aufwand für die Faktorisierung exponentiell mit der Anzahl der Dezimalen von $N$.

Im Jahr 1982 brachte Richard Feynman die interessante Idee auf, auch Computer zu betrachten, die den Gesetzen der Quantenphysik genügen. Zusätzlich zur Interferenz kommt hier die rein quantenmechanische Ressource der Verschränkung zur Anwendung.

In den folgenden Jahren wurden Probleme identifiziert, die mit Hilfe eines solchen Quantencomputers effizienter implementiert werden können. Einer der ersten Quantenalgorithmen war der von Peter Shor im Jahr 1994 entdeckte Algorithmus zur Faktorisierung von Zahlen. Der Aufwand für diesen Algorithmus skaliert nur polynomial im Gegensatz zu allen herkömmlichen Methoden. Eine Realisierung eines Quantencomputers, der große Zahlen mit mehreren hundert Dezimalen faktorisieren könnte, würde daher eine immense Bedrohung für die weltweite Sicherheit der Kommunikation darstellen.

In der vorliegenden Arbeit gehen wir einen alternativen Weg und lösen das Problem der Faktorisierung mit physikalischen Methoden. Dabei beruht unser Ansatz lediglich auf Interferenz und verzichtet auf Verschränkung. Der Schlüssel zu unserem Ansatz besteht in den so genannten Gauß Summen, welche in der Zahlentheorie von großer Bedeutung sind. Zur Faktorisierung nutzen wir speziell die periodischen Eigenschaften von Gauß Summen. Aus physikali- 
scher Sicht liegt der Ursprung der Gauß Summe in der Superposition von Quantenpfaden, welche mit quadratischen Phasenfaktoren gewichtet sind.

Wir entwickeln verschiedene Methoden, um Informationen über die Teiler einer in Parametern des physikalischen Systems codierten Zahl $N$ zu gewinnen. Desweiteren diskutieren wir verschiedene quantenoptische Realisierungen und untersuchen diese hinsichtlich der experimentellen Anforderungen. Alle Realisierungen beruhen auf laserinduzierten Übergängen in Leitersystemen. Die daraus resultierenden Anregungswahrscheinlichkeiten sind proportional zu Gauß Summen, welche experimentell durch Fluoreszenzmessungen gut zugänglich sind.

Bei zwei Vorschlägen induzieren schwache gechirpte Laserpulse Übergänge in speziellen Leitersystemen mit äquidistanten Zuständen. Die gesamte Anregungswahrscheinlichkeit entsteht durch Superposition von verschiedenen Anregungspfaden. In beiden Fällen kommen die quadratischen Phasenfaktoren durch die gechirpten Pulse zustande.

Im Gegensatz dazu kommt unser letzter Vorschlag ohne gechirpte Pulse aus. Die Resonanzbedingung eines Ein-Photonen Übergangs wird linear mit der Zeit variiert. Simultan wird dieser Übergang mit einer Sequenz aus kurzen Pulsen angeregt. Die resultierende Übergangswahrscheinlichkeitsamplitude hat wiederum die Form einer Gauß Summe. Hier ist jedoch die lineare zeitliche Änderung der Resonanzbedingung für die quadratischen Phasen verantwortlich.

Für jeden unserer Vorschläge weisen wir das Potential zur Faktorisierung anhand von numerischen Simulationen nach.

Schließlich diskutieren wir eine experimentelle Realisierung eines Faktorisierungsansatzes, welcher eng an unseren letzten Vorschlag angelehnt ist. Mit Methoden der Kernresonanz Spektroskopie (NMR) ist es vor kurzem Prof. Michael Mehring (Universität Stuttgart) gelungen, eine sechsstellige Zahl ( $N=$ 157573) mit Hilfe spezieller Radiofrequenz Pulssequenzen zu faktorisieren. 


\section{Danksagung}

An dieser Stelle möchte ich die Gelegenheit nutzen und mich bei all jenen bedanken, die mich während meiner Promotion unterstützt haben.

Herrn Professor Wolfgang P. Schleich danke ich besonders für die freundliche Aufnahme in seine Abteilung und für das reizvolle Thema aus dem fächerübergreifenden Gebiet der Atom- und Quantenphysik einerseits und der Mathematik andererseits. Ich bewundere seine Intuition, Verbindungen zwischen scheinbar weit voneinander entfernten Themen herzustellen, sowie seine Begabung, komplizierte Probleme auf das Wesentliche zurückzuführen. Besonders durch unsere zahlreichen, von "Bayern 4 Klassik" untermalten, Schreibsitzungen konnte ich viel von seinem fundierten Wissen über spezielle Funktionen und der Kunst der Näherungsmethoden profitieren. Desweiteren bin ich sehr froh, dass ich mehrfach die Chance hatte, meine Arbeit bei vielen interessanten Konferenzen zu vertreten. Natürlich danke ich Herrn Schleich auch für zahlreiche Einladungen zum Steakessen bei John Benton's.

Auch möchte ich an dieser Stelle Kathy Dodson-Schleich herzlich danken, weil sie sich stets um eine sehr gute Atmosphäre am Institut bemüht und sich immer sehr für stimmungsvolle Weihnachtsfeiern engagiert. Den Sekretärinnen Frau Barbara Casel und Frau Wilma Fiebelkorn danke ich für Ihre Hilfsbereitschaft und Unterstützung bei administrativen Angelegenheiten.

Herrn Professor Ferdinand Schmidt-Kahler gilt mein herzlicher Dank für die Übernahme der Zweitbegutachtung und seine konstruktiven Kritikpunkte, die sicher zur Verbesserung meiner Arbeit beigetragen haben. Für die Bereitschaft bei meinem Promotionskolloquium teilzunehmen, danke ich dem Vorsitzenden des Promotionsausschusses Herrn Professor Frank Steiner. In diesem Zusammenhang danke ich auch Frau Professor Ute Kaiser und Herrn apl. Professor Matthias Freyberger.

Der Landesstiftung Baden Württemberg danke ich für die finanzielle Unterstützung meiner Arbeit im Rahmen des „Quantum Information Highway A8“. 
Mein besonderer Dank gebührt Herrn Professor Gerhard Paulus, der mit seinen stimulierenden Ideen viele neue und interessante Perspektiven eröffnet hat. Many thanks go to Professor Betrand Girard for his support and advice in the context of experimental implementations using chirped pulses. Herrn Professor Helmut Maier danke ich für seine anregende Vorlesungsreihe über die Riemann Zeta Funktion im Sommer 2005.

I'm very grateful to Professor Ilya Sh. Averbukh and Professor Bruce W. Shore for their interest in our work and for most refreshing and inspiring ideas and comments. It was always a pleasure to cooperate with both of them.

Many thanks go to Professor Stig Stenholm for his neat idea of visualizing the the scaling property intrinsic to Gauss sums. I would also like to take the opportunity and thank Profesor M. Suhail Zubairy for his stimulating feed-back from an alternative point of view.

Ganz herzlich danke ich Professor Michael Mehring für sein tiefes Interesse an unserer Arbeit, welches zu einem NMR-Experiment zur Faktorisierung geführt hat. In diesem Zusammenhang möchte ich auch Professor Dieter Suter danken für die Überlassung der Messdaten und nähere Informationen zu seinem alternativen Experiment.

Frau PD Dr. Giovanna Morigi und Dr. Victor V. Kozlov danke ich besonders für die fachliche Betreuung in der ersten Phase meiner Arbeit. Sie nahmen sich stets Zeit für meine Fragen und Diskussionen. An dieser Stelle möchte ich mich auch bei den Herrn apl. Professor Matthias Freyberger, Dr. Karl Vogel, PD Dr. Reinhold Walser und Dr. Eric Lutz für wertvolle Tips aus ihrem vielfältigen Erfahrungsschatz bedanken.

I would also like to express my sincere thanks to Martin Štefaňák and Daniel Haase for the interesting perspectives and successful cooperation during my last months at the institute.

Ein ganz herzlicher Dank geht an Martin Štefaňák, Bernd Mohring, Gerrit Nandi und nicht zuletzt an Alexander Wolf für das kritische Durchsehen meiner Arbeit und für konstruktive Verbesserungsvorschläge. Für ihre Unterstützung im Rahmen meines Promotionsverfahrens danke ich Daniela Denot ganz besonders.

Bei auftretenden Computerproblemen, aber auch bei physikalischen Problemen haben mir Holger Mack, Bernd Mohring, Stefan Probst und Rüdiger Mack stets bereitwillig ausgeholfen.

Ganz besonders möchte ich mich bei meinen Zimmerkollegen Marc Bienert, Oliver Crasser, Aldo Delgado, Hannah Venzl und Carsten Weiß für eine nette Atmosphäre danken. Marc danke ich auch für seine Gastfreundschaft während meines Besuchs in Cuernavaca, Mexiko.

Mein Dank gebührt meiner Familie für die jahrelange Unterstützung. Zu guter Letzt möchte ich mich ganz herzlich bei meiner Partnerin Ingrid Rabeder für ihr großes Verständnis und ihre liebevolle Unterstützung in all den Jahren bedanken. 


\section{Curriculum Vitae}

Name: Wolfgang Merkel

Address: $\quad$ Emanuelstr. 10

89231 Neu-Ulm

Date of Birth: $\quad$ May, 1st, 1974

Place of Birth: Heidenheim/Brenz

School Education

09/1980 - 07/1984 Grundschule Hürben

09/1984 - 06/1993 Werkgymnasium Heidenheim/Brenz

\section{Civilian Sevice}

07/1993 - 09/1994 Abteilung für Nuklearmedizin, Universitätsklinikum Ulm

\section{University Education}

10/1994 - 08/1996 Study of physics at the Universität Ulm

09/1996 - 06/1997 Study of physics at the University of Leeds, England

10/1997 - 10/2001 Study of physics at the Universität Ulm

01/2002 -01/2007 Scientific employee at the Institut für Quantenphysik, Universität Ulm 


\section{List of Publications}

1. M. Bienert, W. Merkel and G. Morigi,

"Resonance fluorescence of a trapped three-level atom", Phys. Rev. A 69, p. 013405-1-12 (2004).

2. M. Bienert, W. MERKEL and G. Morigi, "Inelastic scattering of light by a cold trapped atom: Effects of the quantum center-of-mass motion", Phys. Rev. A 73, p. 033402 (2006).

3. W. Merkel, O. Crasser, F. Haug, E. Lutz, H. Mack, M. Freyberger, W. P. Schleich, I. Sh. Averbukh, M. Bienert, B. Girard, H. Maier and G. G. PAUlus, "Chirped pulses, Gauss sums and the factorization of numbers", Int. J. of Mod. Phys. B 20, Nos. 11-13, p. 1893-1916 (2006).

4. W. Merkel, I. Sh. Averbukh, B. Girard, G. G. Paulus and W. P. SCHLEICH, "Factorization of numbers with physical systems", Fortschritte der Physik 54, Nos. 8-10, p. 856-865 (2006).

This article appeared slightly modified in "Elements of Quantum Information", edited by W. P. Schleich and H. Walther (Wiley VCH, Berlin, 2007)

5. M. Mehring, K. Müller, I. Sh. Averbukh, W. Merkel, and W. P. SCHLEICH, "NMR Experiment factors numbers with Gauss sums", Phys. Rev. Lett. 98, p. 120502 (2007), e-print: quant-ph/0609174.

6. W. Merkel, H.Mack, M. Freyberger, V. V. Kozlov, B. W. Shore and W. P. SCHLEICH, 
"Coherent transport of single atoms in optical lattices", Phys. Rev. A 75, p. 033420 (2007).

7. W. Merkel, H.Mack, E. Lutz, B. Girard, G. G. Paulus and W. P. SCHLEICH, "Chirping a two-photon transition in a multistate ladder", Phys. Rev. A , accepted (2007), e-print: quant-ph/0702122.

8. M. ŠTtefaňÁK, W. MERKEL, W. P. Schleich, D. HAASE Und H. MAIER, "Optimal truncation of Gauss sums for integer factorization", in preparation for N. J. P. (2007). 